\title{
Drop impact tester development for spent nuclear fuel vibration integrity study
}

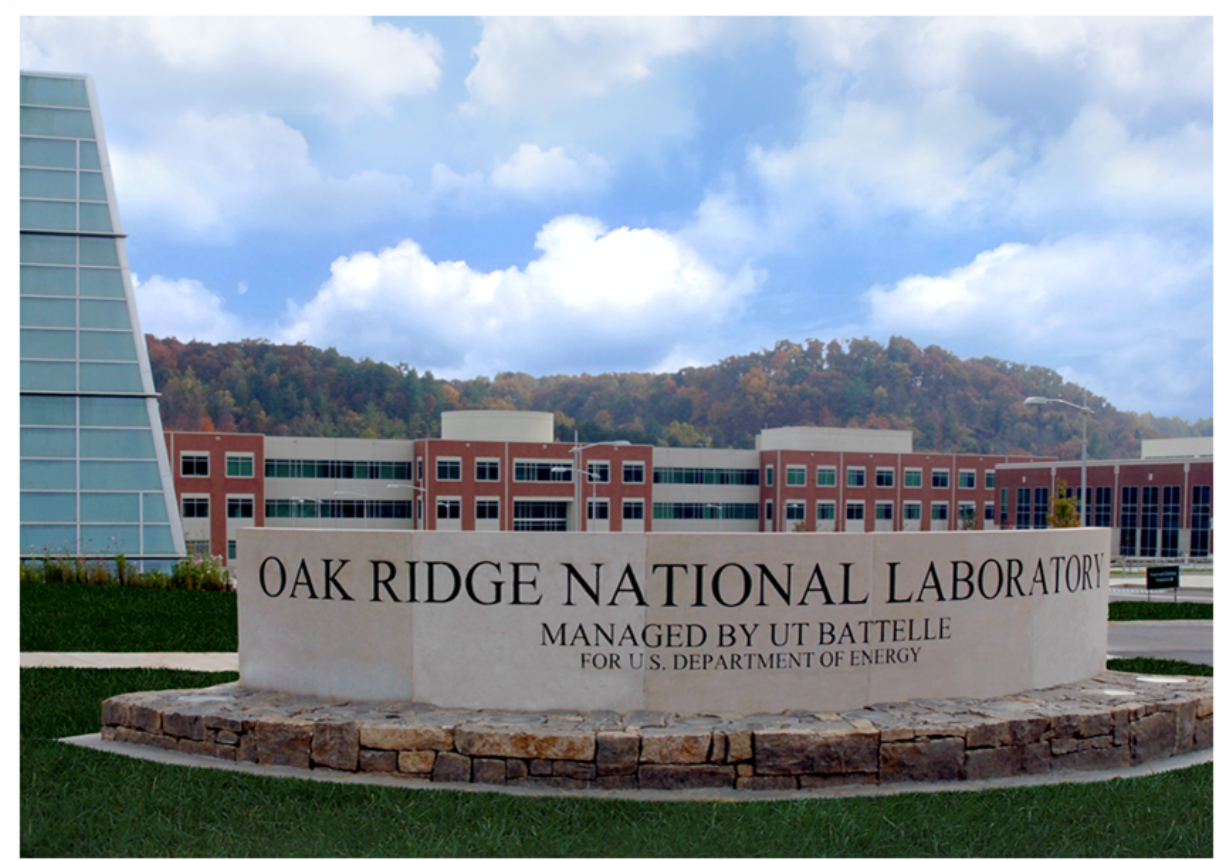

Jy-An Wang

Rick R. Lowden

Rose Montgomery

Bruce Bevard 


\section{DOCUMENT AVAILABILITY}

Reports produced after January 1, 1996, are generally available free via US Department of Energy (DOE) SciTech Connect.

Website www.osti.gov

Reports produced before January 1, 1996, may be purchased by members of the public from the following source:

National Technical Information Service

5285 Port Royal Road

Springfield, VA 22161

Telephone 703-605-6000 (1-800-553-6847)

TDD 703-487-4639

Fax 703-605-6900

E-mail info@ntis.gov

Website http://classic.ntis.gov/

Reports are available to DOE employees, DOE contractors, Energy Technology Data Exchange representatives, and International Nuclear Information System representatives from the following source:

Office of Scientific and Technical Information

PO Box 62

Oak Ridge, TN 37831

Telephone 865-576-8401

Fax 865-576-5728

E-mail reports@osti.gov

Website http://www.osti.gov/contact.html

This report was prepared as an account of work sponsored by an agency of the United States Government. Neither the United States Government nor any agency thereof, nor any of their employees, makes any warranty, express or implied, or assumes any legal liability or responsibility for the accuracy, completeness, or usefulness of any information, apparatus, product, or process disclosed, or represents that its use would not infringe privately owned rights. Reference herein to any specific commercial product, process, or service by trade name, trademark, manufacturer, or otherwise, does not necessarily constitute or imply its endorsement, recommendation, or favoring by the United States Government or any agency thereof. The views and opinions of authors expressed herein do not necessarily state or reflect those of the United States Government or any agency thereof. 
Materials Science and Technology Division

Drop impact tester development for spent nuclear fuel vibration integrity study

Jy-An Wang, Rick R. Lowden

\section{Program Manager}

Rose Montgomery ${ }^{\mathrm{a}}$, Bruce Bevard ${ }^{\mathrm{a}}$

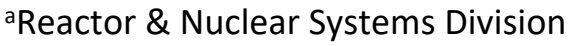

Date Published: February 2021

Prepared by

OAK RIDGE NATIONAL LABORATORY

Oak Ridge, TN 37831-6283

managed by

UT-BATTELLE, LLC

for the

US DEPARTMENT OF ENERGY

under contract DE-AC05-00OR22725 
This page is intentionally blank 


\section{Contents}

ABSTRACT vii

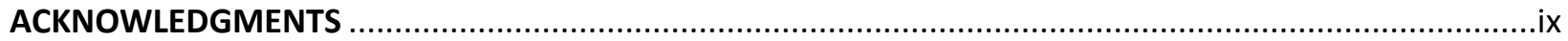

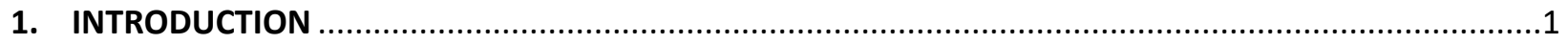

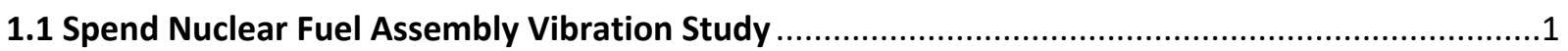

1.2 Fuel Assembly System Contact Transient Shock Study at Spacer Grid Region ...............................

1.2.1 FEA simulation results for SNF rod and spacer grid system dynamic contact interaction ...........5

1.2.2 FEA simulation results for drop contact interaction of SNF rod and projectile system with

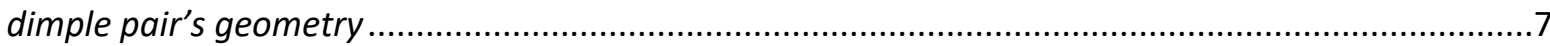

2. ACCUMULATE DAMAGE EVALUATION FOR SPENT FUEL UNDER NCT ......................................

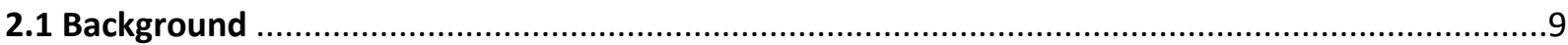

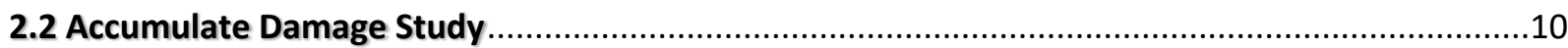

2.3 Drop Impact Tester Design Concepts and Accumulated Damage Evaluation .............................10

2.4 Drop Impact Tester Design Configurations \& Development ....................................................13

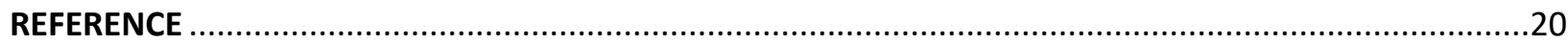

APPENDIX A: DYNAMIC DEFORMATION SIMULATIONS OF SPENT NUCLEAR FUEL ASSEMBLY [1] .........21

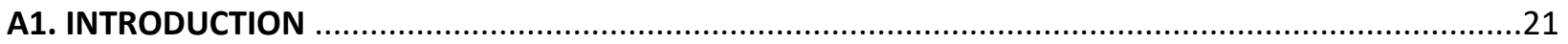

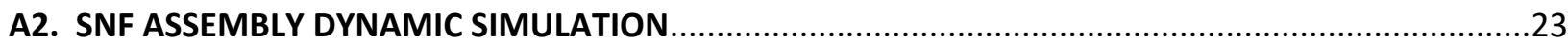

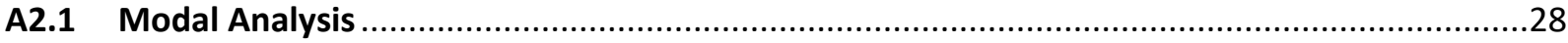

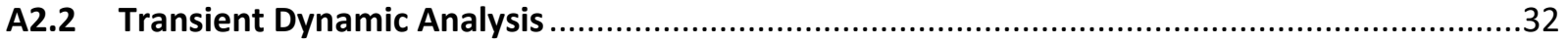

APPENDIX B: EVALUATION OF CONTACT INTERACTION BETWEEN FUEL ROD AND SPACER GRIDS [1]..41 


\section{Tables of Figures}

Fig. 1. Schematic of $17 \times 17$ PWR fuel assembly.

.2

Fig. 2. SNF subassembly model for normal transportation evaluation.

Fig. 3. Gravity loads for the initial stage of transient dynamic simulation of the fuel subassembly model.

Fig. 4. Cross section view of the fuel rod with two dimples, modeled within the spacer grid region. .....5

Fig. 5. Cross section view of the fuel rod with the leaf spring modeled at the spacer grid region. ..........5

Fig. 6. Segment 1 accelerometer time-histories [8], where sensor A7 is located at spacer grid..............6

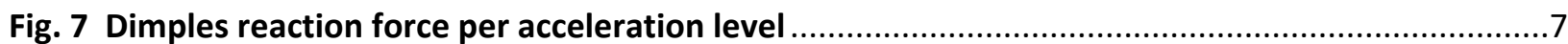

Fig. 8 Four dimple-pair head projectiles designed with weights of $355 \mathrm{~g}, 310 \mathrm{~g}, 83 \mathrm{~g}$, and $47 \mathrm{~g}$, from

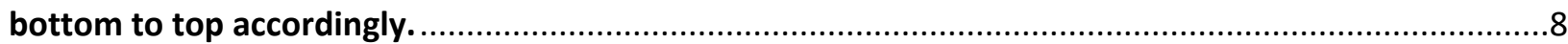

Fig. 9 FEA simulation of modified CIRFT sample with weight matched that of targeted projectile.........8

Fig. 10 Drop impact contact reaction force per drop height............................................................

Fig. 11. CIRFT - Cyclic Integrated Reversible-bending Fatigue Tester .............................................10

Fig. 12 Methodology used for estimating SNF assembly system vibration effective lifetime study.......12

Fig. 13 The schematic diagram of conducting random-frequency response analyses........................13

Fig. 14 Schematic diagram of drop impact tester design with a top-mounted stepper motor for lifting projectile.

Fig. 15 (Left) impact shaft and specimen holder, (Right) is the close look of specimen holder pulled out of impact chamber. The clearance between impact projectile exit and the bottom the supporting plate is about 1-1/4". The clearance between specimen rod (2-inch gage section) and specimen holder is about 0.3".

Fig. 16 Line-up view of CIRFT specimen and bottom of specimen holder.

Fig. 17 (Left) The second generation drop impact tester, where the mechanical lifter is located on the bottom of the moving track; (Right) The first generation drop impact tester, where the mechanical lifter is located on the top of the moving track.

Fig. 18 Close view of the loading train for floor mounted driver, including an L-angle connector for magnet driver and stepper motor wrings for a hot-cell implementation.

Fig. 19 Drop impact tester hot-cell implementation (a) wire length increased to 25 feet, and (b) lifting plate with leveling functionality was added to the drop impact tester for hot-cell installation. 


\begin{abstract}
The accumulated damage of SNF rod is defined as the integrated damage induced by the SNF assembly harmonic vibrations and SNF assembly \& cask system contact interactions induced transient shocks, experienced by SNF rods during normal conditions of transportation (NCT).

The CIRFT technology has provided means to resolve/understand the SNF rod harmonic vibration reliability issues associated with maximum axial clad tubing stress induced by SNF inertia related dynamic bending loading. However, the SNF rod integrity in association with the transient shock loads during NCT through dynamic contact interactions among SNF assembly cannot be investigated systematically through CIRFT technology alone. Thus, the proposed drop impact tester development become a viable tool for SNF system transient shock effect investigation.

Furthermore, the transient shocks induced loading mode is normally perpendicular to the SNF rod axial orientation, i.e., in a transverse orientation to the SNF rod. To proceed with such loading mode investigation, a new device, "SNF Drop Impact Tester" that provides the transverse impact shock load onto a SNF rod through a free-drop projectile was developed in FY20 for SNF System Vibration Reliability Investigation; where the SNF system contact impact intensity can be calibrated accordingly with projectile weight and projectile head geometry.
\end{abstract}

This newly developed research tool can further provide a detailed understanding about the effect of dynamic contact-interaction loadings; and combined harmonic vibration and transient shock loading modes on the fatigue damage evolution of the HBU SNF under NCT. 
This page is intentionally blank 


\section{ACKNOWLEDGMENTS}

This research was jointly sponsored by the US Department of Energy (DOE) Used Fuel Disposition Campaign (UFDC) under DOE contract DE-AC05-000R22725 with UT-Battelle, LLC. The authors thank ORNL program managers, Rose Montgomery and Bruce Bevard, for their continued support and guidance during the program development, Hong Wang and Lianshan Lin for reviewing the report. 
This page is intentionally blank 


\section{INTRODUCTION}

\subsection{Spend Nuclear Fuel Assembly Vibration Study}

Random vibration registered at the SNF transport cask, which is excited from a railcar bed, provides the external loading driver to vibrate the SNF assembly. In addition to this external vibration driver, the fuel assembly also registers internal transient shocks resulting from the dynamic interactions among the fuel assembly components inside the cask. These components include the skeleton, fuel rods, and canister basket walls (Fig. 1).

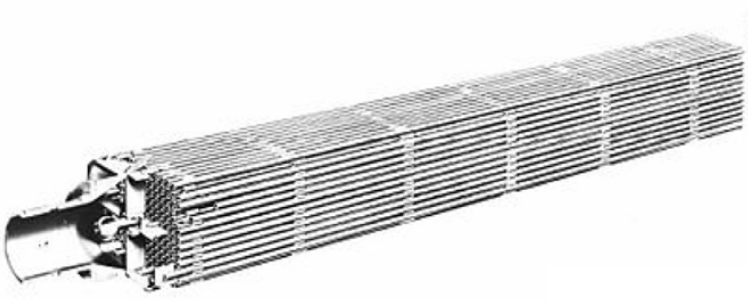

Fuel assembly
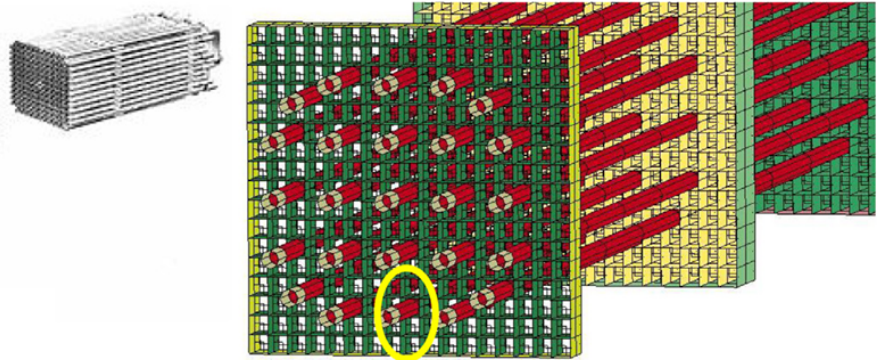

Guide tubes and spacer grids [2]

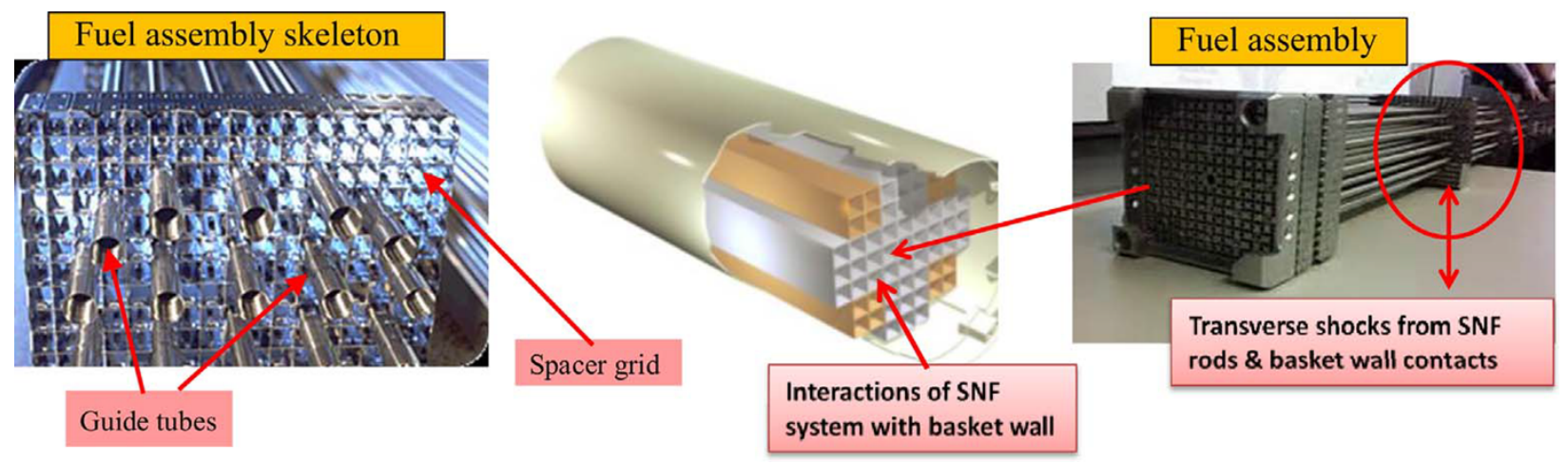

Fig. 1. Schematic of $17 \times 17$ PWR fuel assembly.

In a horizontal layout of a spent nuclear fuel (SNF) assembly under normal conditions of transportation (NCT), the fuel assembly's skeleton formed by guide tubes and spacer grids is the primary load bearing structure for carrying and transferring the vibration loads within an SNF assembly. Therefore, the integrity of guide tubes and spacer grids will dictate the vibration amplitude/intensity of the fuel assembly during transport and must be considered when designing multipurpose canister (MPC) for safe SNF transport.

The research focuses on the targeted SNF subassembly deformation dynamics that can occur during normal vibration mode, as well as the cask's internal transient shock mode during NCT. $[1,2]$ The two fuel rods with a guide tube, the spacer grids (Fig. 2) at the bottom edge of full fuel assembly, and the partial basket wall were modeled to investigate interaction of fuel assembly components, as well as interaction against the basket wall or the spacer grids. Each fuel rod was modeled with the actual dimensions of 240 fuel pellets in a 3.658-m-long fuel cladding. The fuel subassembly model contains 
numerous contact conditions and represents itself as a highly nonlinear system. The transient dynamic analyses were performed using ABAQUS-explicit code in the time domain for nonlinear system evaluation. The details of subassembly FEA results are provided in Appendix A.

There are 264 fuel rods contained in the spacer grid slots because there are 25 guide tubes for a $17 \times 17$ fuel assembly. The guide tube modeled at the edge of the full SNF assembly will roughly carry the gravity load of the neighboring 10 fuel rods through the spacer grids. A gravitational acceleration loading of 9.8 $\mathrm{m} / \mathrm{s}^{2}$ is applied to all components. The gravitation loads from the remaining 8 fuel rods are applied as the concentrated loads evenly distributed to eight spacer grids, as shown in Fig. 3. The developed dynamic simulation protocol consists of two stages. During the first stage, the basket wall is constrained, while the assembly (mainly the intermediate spacer grids) sags down due to the gravity load and comes to rest on the basket floor. During the second stage, the acceleration with a sine wave form or impulse signal to represent the normal vibration excitation or transient shock excitation that was applied to the basket and nozzles for transient dynamic analyses. In the second transient excitation stage, the constraint at the basket floor is removed, but the gravity loads are maintained for both stages of simulations. Therefore, the procedure for the subassembly model includes an initialization stage to establish gravity-induced inter-contacting followed by a transient dynamic loading stage.

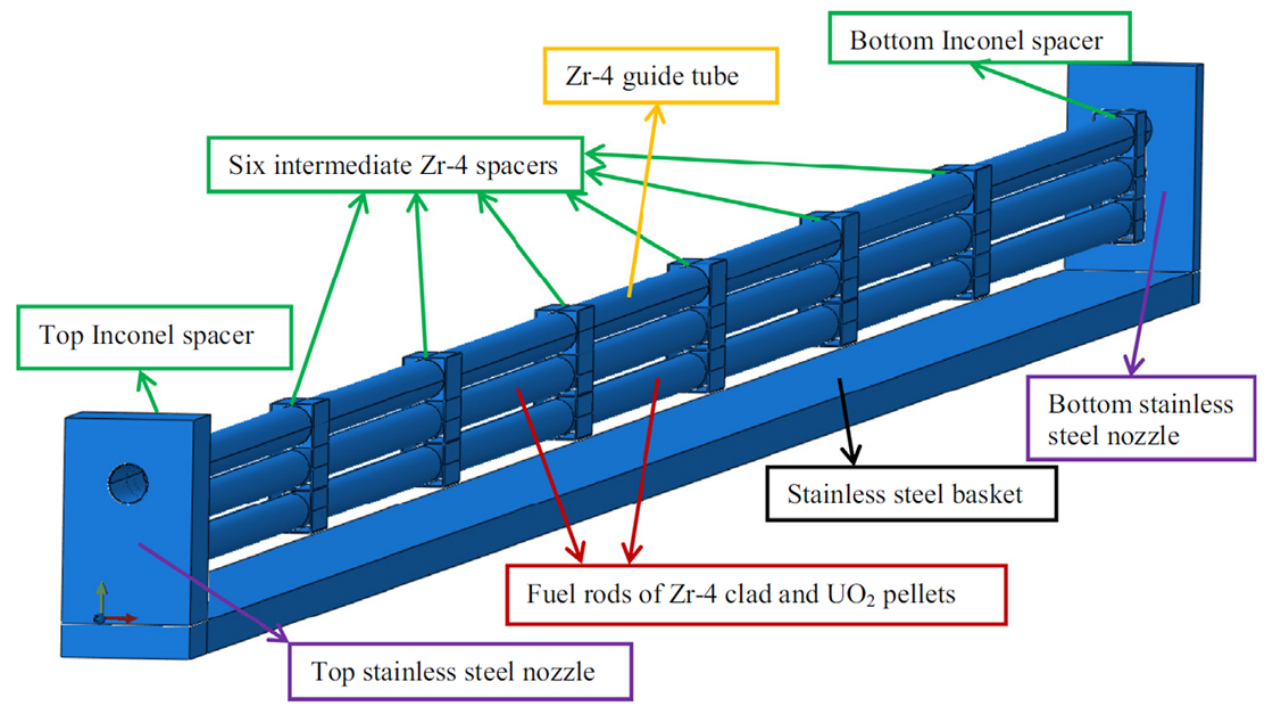

Fig. 2. SNF subassembly model for normal transportation evaluation. 


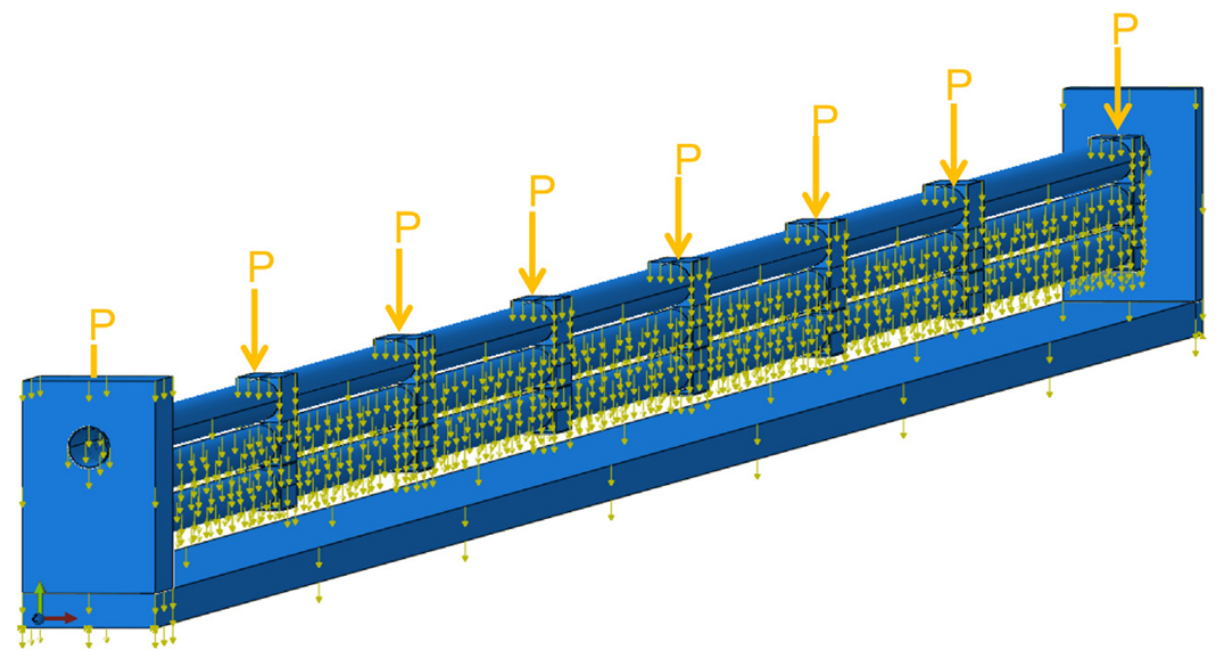

Fig. 3. Gravity loads for the initial stage of transient dynamic simulation of the fuel subassembly model.

Dynamics analyses were performed in the frequency domain to gain a better understanding of the frequency characteristics of the fuel assembly system and in the time domain to simulate the transient dynamic response of the fuel assembly. FEA simulation results are used to determine the stress and strain states of subassembly model components such as fuel rods, the guide tube, spacer grids, nozzles, and the basket wall under vibration loading during normal vibration conditions and transient shocks during NCT. The resulting stress/strain data can be used in future fuel assembly component failure analyses. The contact interactions between system components during transient dynamic simulation were investigated.

Their dynamic interactions can significantly increase the high rate impact loading frequency within fuel assembly components during NCT. Sandia National Laboratories (SNL) registered maximum $22 \mathrm{~g}$ peak vertical vibration acceleration at mid span of the surrogate rod adjacent to the spacer grid from the accelerometer reading during the truck transportation test [3]. In contrast, the maximum vertical acceleration of $5.6 \mathrm{~g}$ was registered on the top of transport basket at mid-span.

\subsection{Fuel Assembly System Contact Transient Shock Study at Spacer Grid Region}

To further evaluate the intensity of contact interaction induced by the local contacts' impact loading at the spacer grid, detailed models of the actual spring and dimples of the spacer grids were created.

Fig. 4 and Fig. 5 provide the details of a 3-D model of the fuel rod section with the dimples and leaf spring structures at the spacer grids. The fuel rod length is equivalent to a quarter of the length between the spacer grids. According to the targeted spacer grid design, there are two dimples on one side of a slot and one spring on the opposite side of the slot. 

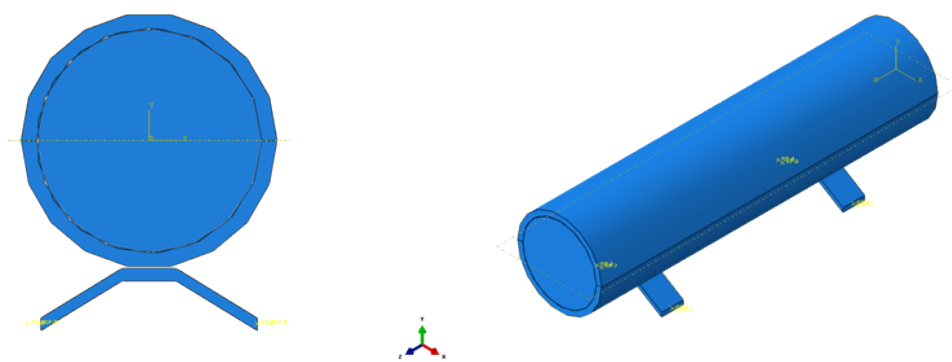

Fig. 4. Cross section view of the fuel rod with two dimples, modeled within the spacer grid region.

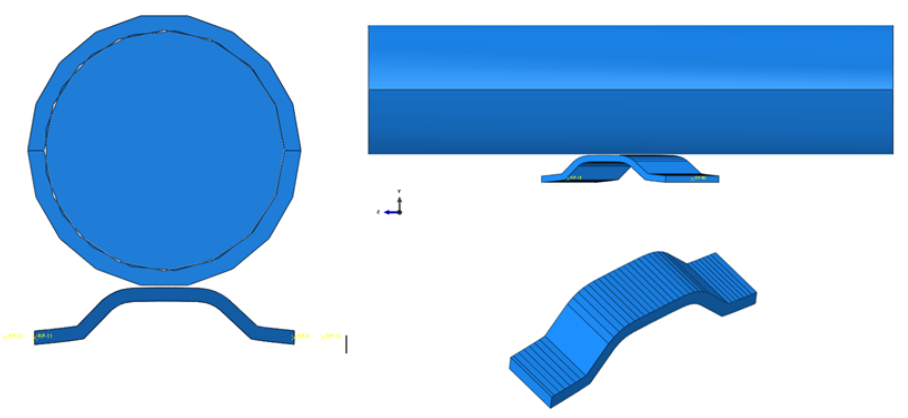

Fig. 5. Cross section view of the fuel rod with the leaf spring modeled at the spacer grid region.

The impacts between the fuel rod and springs and dimples were simulated with a $20 \mathrm{~g}$ transient shock load, obtained from Sandia truck road test shown in Fig. 6. The associated contact interaction intensities, in terms of reaction forces, were estimated from the finite element analyses results. The detailed FEA simulation of transient shock at spacer grid region was provided in Appendix B. 


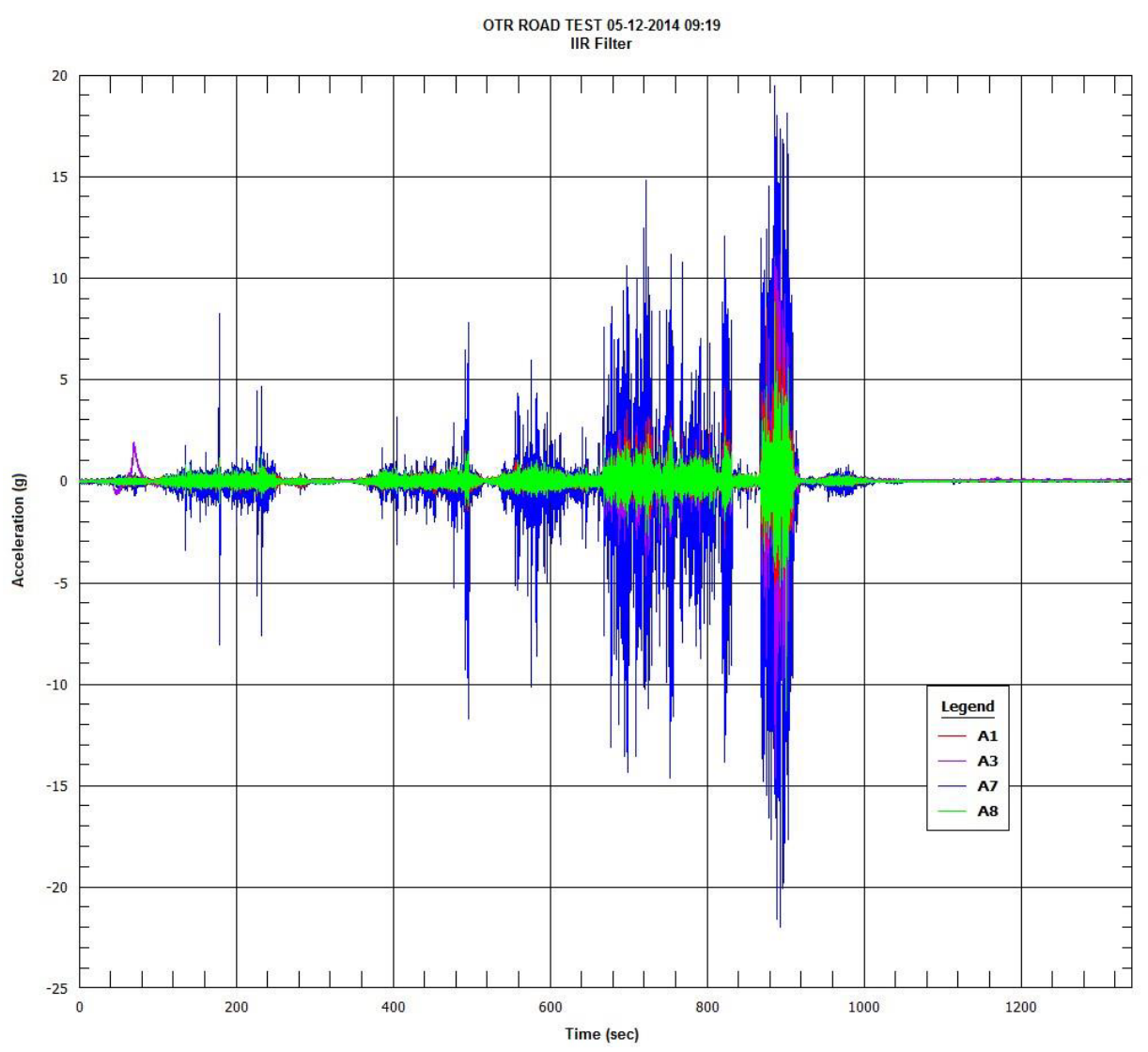

Fig. 6. Segment 1 accelerometer time-histories [8], where sensor A7 is located at spacer grid [3].

To estimate the damage potential of the transient shock to the SNF vibration fatigue lifetime, a-foot drop tests were performed on the CIRFT specimens. FEA was also used to investigate the contact reaction intensity level onto CIRFT test samples during impact loading induced by drop tests. The test results of combined Drop-impact and CIRFT tests show reduced fatigue lifetime from SNF CIRFT specimens [2]. Furthermore, the 1-foot drop of CIRFT sample induced maximum SNF rod contact stress profile was close to that of a $20 \mathrm{~g}$ acceleration transient shock load at the spacer grid region. Moreover, the contact reaction force at clad surface is also a good index to be used to link/define the drop impact load intensity that can produce compatible reaction force experienced by SNF clad under the transient shock load induced by SNF assembly contact interactions.

\subsubsection{FEA simulation results for SNF rod and spacer grid system dynamic contact interaction}

The FEA model design and its limit criteria are stated as following:

(1) SNF rod length of $1 / 4$ distances between spacer grids was used as SNF mass involved in dynamic contact interaction evaluation.

(2) Spacer grid failure was not considered during simulation at higher acceleration level.

(3) Nonlinear geometry deformation of dimple or spring system has significant impact on the reaction force evaluation as shown in Fig. 7. 
(4) Therefore, the developed reaction force per acceleration level shown in Fig. 7 is only strictly applied to the SNF rod and spacer grid contact interaction scenario without significant dimples damages or failures; and does not applied to other hard contact interaction phenomena, such SNF rod and basket wall contact interaction or SNF rod to SNF rod contact interaction, etc.. The referred hard contact interaction of SNF assembly would require sub-assembly FEA model with full SNF rod length.

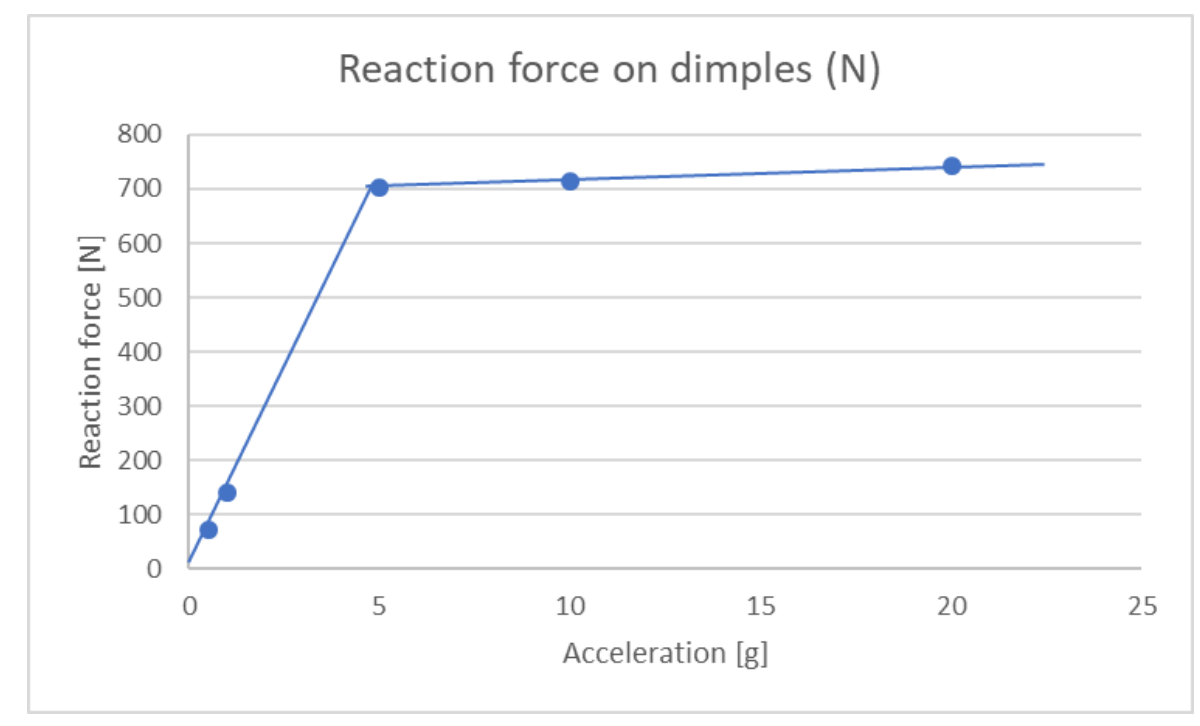

Fig. 7 Dimples reaction force per acceleration level

In order to carry out SNF accumulated damage study utilizing drop impact test approach, drop impact FEA was performed to build linking mechanism between SNF vibration and drop impact testing that can resemble either (1) spacer grid/SNF rod contact interaction, or (2) SNF rod/basket wall or SNF rod/SNF rod hard contact interaction. The current projectile used is designed to mimic dimples impact effect at spacer grid region. There are 4 projectiles, with weights of $355 \mathrm{~g}, 310 \mathrm{~g}, 83 \mathrm{~g}$, and $47 \mathrm{~g}$, were designed accordingly to mimic dimples structure contact impact effect, as shown in Fig. 8: 


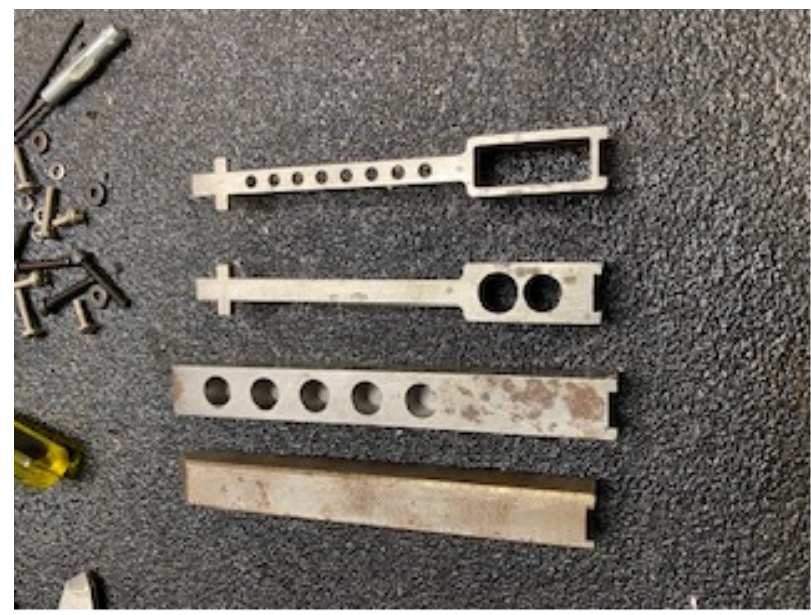

Fig. 8 Four dimple-pair head projectiles designed with weights of $355 \mathrm{~g}, 310 \mathrm{~g}, 83 \mathrm{~g}$, and $47 \mathrm{~g}$, from bottom to top accordingly.

1.2.2 FEA simulation results for drop contact interaction of SNF rod and projectile system with dimple pair's geometry

The dynamic input of drop impact with $83 \mathrm{~g}$ projectile are the following: (1) Abaqus explicit dynamic code was used, where the impact time frame for 3 inch drops is set as 0.002 second, 6 inch drops is set as 0.0012 second, while 9 and 12 inch drops is set at 0.001 second. The initial dynamic condition is set to initial contact velocity at drop impact contact instant. FEA model two dumbbell weights density can be varied to produce total weight that match the selected projectile weight, see Fig. 9.

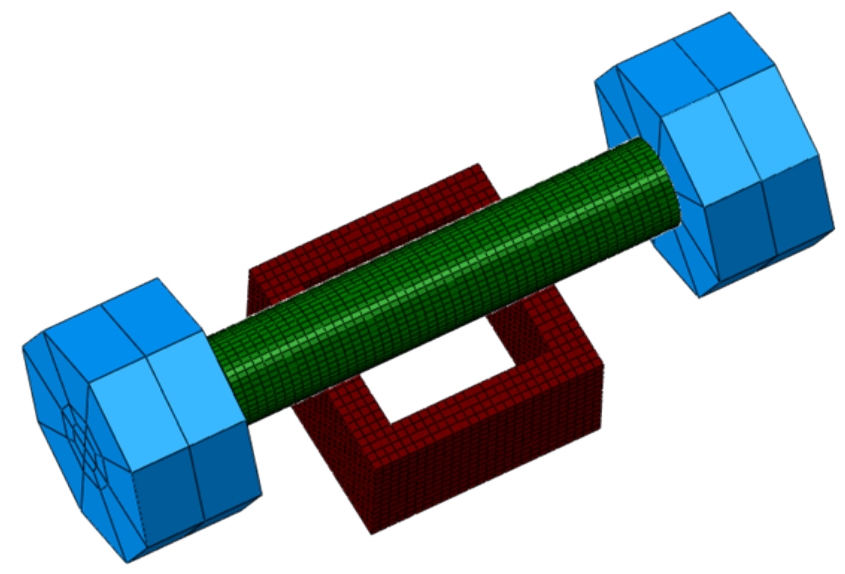

Fig. 9 FEA simulation of modified CIRFT sample with weight matched that of targeted projectile. 
The FEA simulated reaction forces at drop contact interface for projectiles $310 \mathrm{~g}$ and $83 \mathrm{~g}$ are shown in Fig. 10, where $47 \mathrm{~g}$ projectile at 6-inch drop FEA simulation was also provided for comparison purpose.

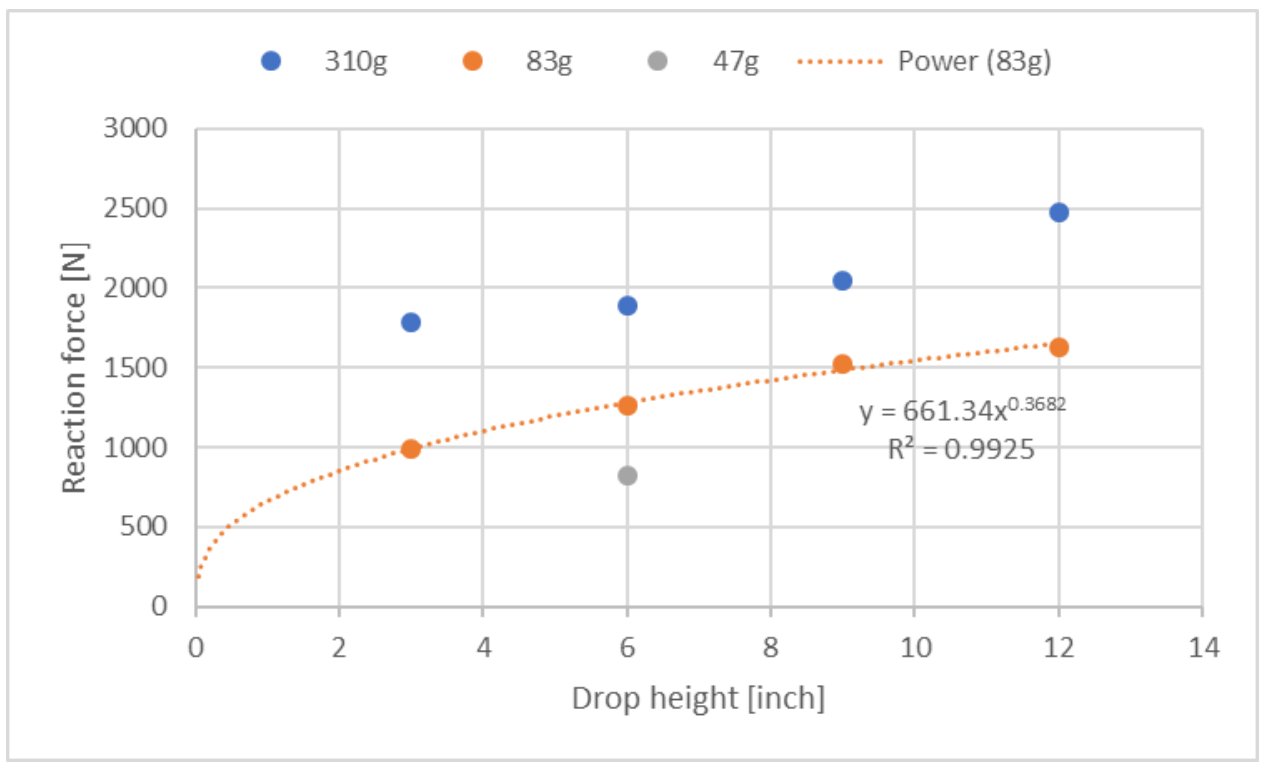

Fig. 10 Drop impact contact reaction force per drop height

From Fig. 7, the dimple reaction force on the clad surface appeared to be bi-linear; this means that acceleration above $5 \mathrm{~g}$ level, the dimple reaction force saturated (due to geometry instability such as dimple buckling, or material bi-linear yield behavior).

The estimated reaction force per $3.0 \mathrm{~g}$ and $4.5 \mathrm{~g}$ loads from Fig. 7 are $400 \mathrm{~N}$ and $600 \mathrm{~N}$, respectively. The corresponding drop heights of $83 \mathrm{~g}$ projectile required for reaching $400 \mathrm{~N}$ and $600 \mathrm{~N}$ reaction forces are 0.25 inch and 0.775 inch, respectively. The required drop height appears to be small based on the reaction forces estimated from the $83 \mathrm{~g}$ projectile impact load.

Based on reaction force of $47 \mathrm{~g}$ projectile at 6-inch drop, $821 \mathrm{~N}$, it has $445 \mathrm{~N}$ decrease compared to that of $83 \mathrm{~g}$ projectile at 6 -inch drop height. Extrapolating from reaction force data of 6 -inch drop per $47 \mathrm{~g}$ projectile, the rough estimate of drop height required for $47 \mathrm{~g}$ projectile to meet reaction forces of $3 \mathrm{~g}$ and 4.5 accelerations at spacer grid are 2 inch and 3.5 inch, respectively. Thus, based on projection obtained from $47 \mathrm{~g}$ projectile data with 6-inch drop, it appears that using lighter projectile of 47g has more appropriate drop height than that of $83 \mathrm{~g}$ projectile. Nevertheless, a complete FEA simulation that cover full spectrum of drop height range for $47 \mathrm{~g}$ projectile is recommended. 


\section{ACCUMULATE DAMAGE EVALUATION FOR SPENT FUEL UNDER NCT}

\subsection{Background}

Transportation packages for SNF must meet safety requirements under normal and accident conditions as specified by federal regulations. During road or rail transportation, SNF will experience unique conditions that could affect the structural integrity of the cladding due to vibrational and impact loading. Lack of SNF inertia-induced dynamic fatigue data, especially for the high burn-up (HBU) SNF systems, has brought significant challenges to quantify the reliability of SNF during transportation with a high degree of confidence. To address this shortcoming, Oak Ridge National Laboratory (ORNL) developed a SNF vibration testing protocol without fuel pellets removal, which has provided significant insight regarding the dynamics of mechanical interactions between pellet and cladding [4]. Static and dynamic loading experimental data were generated for SNF under simulated transportation environments using a cyclic integrated reversible-bending fatigue tester (CIRFT, shown in Fig. 11), an enabling hot-cell testing technology developed at ORNL, which has received an US Patent No. 8,863,585 B2 in 2014. SNF flexural tensile strength and fatigue S-N data from pressurized water reactors (PWRs) and boiling water reactor (BWR) HBU SNF are investigated, including the potential effects of pellet-cladding interface bonding, hydride reorientation, and thermal annealing to SNF vibration reliability.

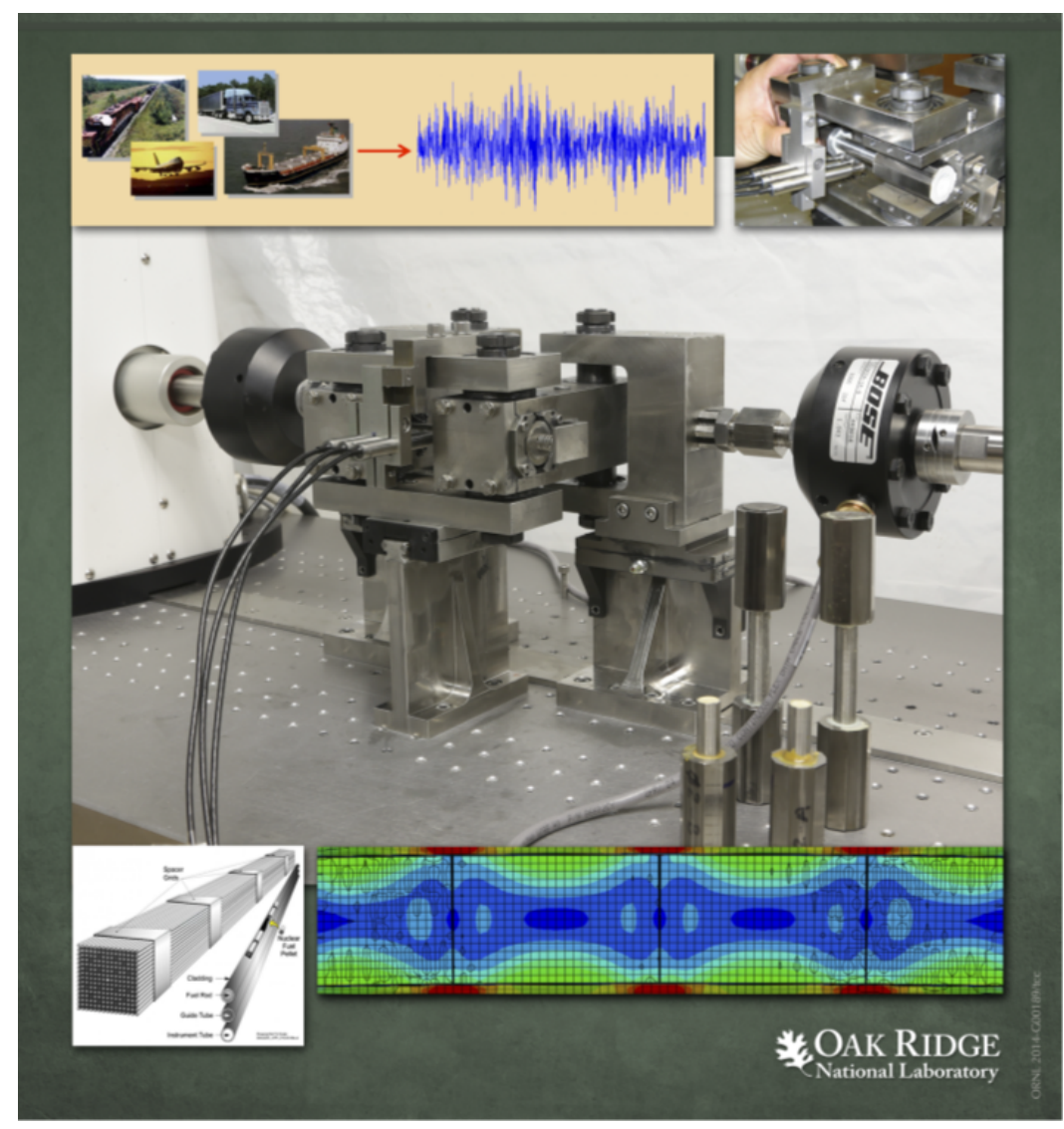

Fig. 11. CIRFT - Cyclic Integrated Reversible-bending Fatigue Tester 


\subsection{Accumulate Damage Study}

The accumulated damage of SNF rod is defined as the integrated damage induced by the SNF assembly harmonic vibrations and SNF assembly \& cask system contact interactions induced transient shocks, experienced by SNF rods during NCT.

The CIRFT technology has provided means to resolve/understand the SNF rod harmonic vibration reliability issues associated with maximum axial clad tubing stress induced by SNF inertia related dynamic bending loading. However, the SNF rod integrity in association with the transient shock loads during NCT through dynamic contact interactions among SNF assembly (including spacer grips and canister basket walls), and the combine effect of transient shocks and the SNF inertia induced dynamic reversible-bending loading has not been investigated systematically. Furthermore, the transient shocks induced loading mode is normally perpendicular to the SNF rod axial orientation, i.e., in a transverse orientation to the SNF rod. To proceed with such loading mode investigation, a new device, "SNF Drop Impact Tester" that provides the transverse impact shock load onto a SNF rod through a free-drop projectile was developed in FY20 for SNF System Vibration Reliability Investigation; where the SNF system contact impact intensity can be calibrated accordingly with projectile weight and projectile head geometry. This newly developed research tool can further provide a detailed understanding about the effect of dynamic contact-interaction loadings; and combined harmonic vibration and transient shock loading modes on the fatigue damage evolution of the HBU SNF under NCT.

\subsection{Drop Impact Tester Design Concepts and Accumulated Damage Evaluation}

The design concept of the proposed drop impact tester is to use free-fall projectile to simulate the dynamic contact reaction forces on the clad surface experienced by the SNF assembly system contact interactions under NCT, including fuel rods, spacer grips, and basket walls. Therefore, the drop impact projectile is designed to simulate similar transient shock force intensity, experienced by SNF system under dynamic contact interactions, with calibrated projectile weight and appropriate projectile head geometry. For instance, to simulate transient shock induced by the dimple structure of the spacer grid to SNF rod, the designed projectile head has two-dimple structure. FEAs were carried out to estimate the contact reaction forces and the clad surface stress profiles of clad-projectile contact interaction, per targeted drop impact loads at various heights. Thus, the contact reaction force or/and contact induced stress profile on the clad surface, estimated from the developed FEA procedure, provides the direct linking mechanism between SNF system contact interaction intensity under NCT and that simulated by drop impact projectile.

The accumulated damage study of SNF assembly can be performed by using both drop impact tester and CIRFT devices, with the following sequences: (1) The drop impact test would be carried out onto a CIRFT sample, (2) and then the impact-aged CIRFT sample would be undergone a normal CIRFT bending testing to induce accumulated damage onto the CIRFT sample. The drop impact test loading cycles performed on a CIRFT sample is designed to match that of the registered transient shocks frequency experienced by the SNF rods system under NCT. The final integrated drop impact and fatigue bending test results will provide the essential info regarding the accumulated damage effect of the SNF system vibration reliability under NCT. 
The methodology of evaluating SNF system vibration induced fatigue evolution and its associated SNF effective lifetime under NCT, through the field vibration data collection and the fuel assembly dynamic response analyses, is illustrated in Fig. 12. The schematic diagram of conducting random-frequency response analyses is illustrated in Fig. 13.

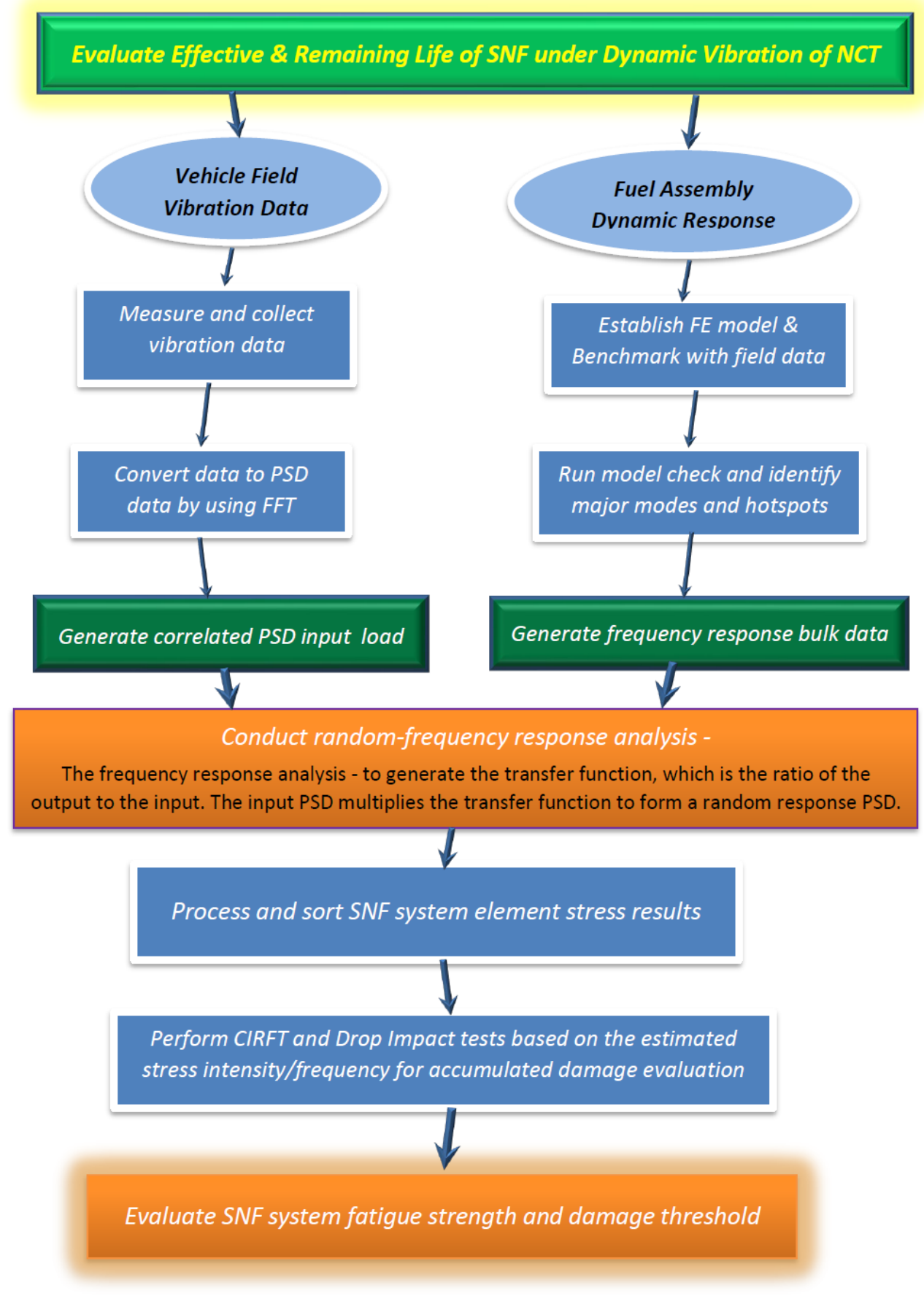

Fig. 12 Methodology used for estimating SNF assembly system vibration effective lifetime. 


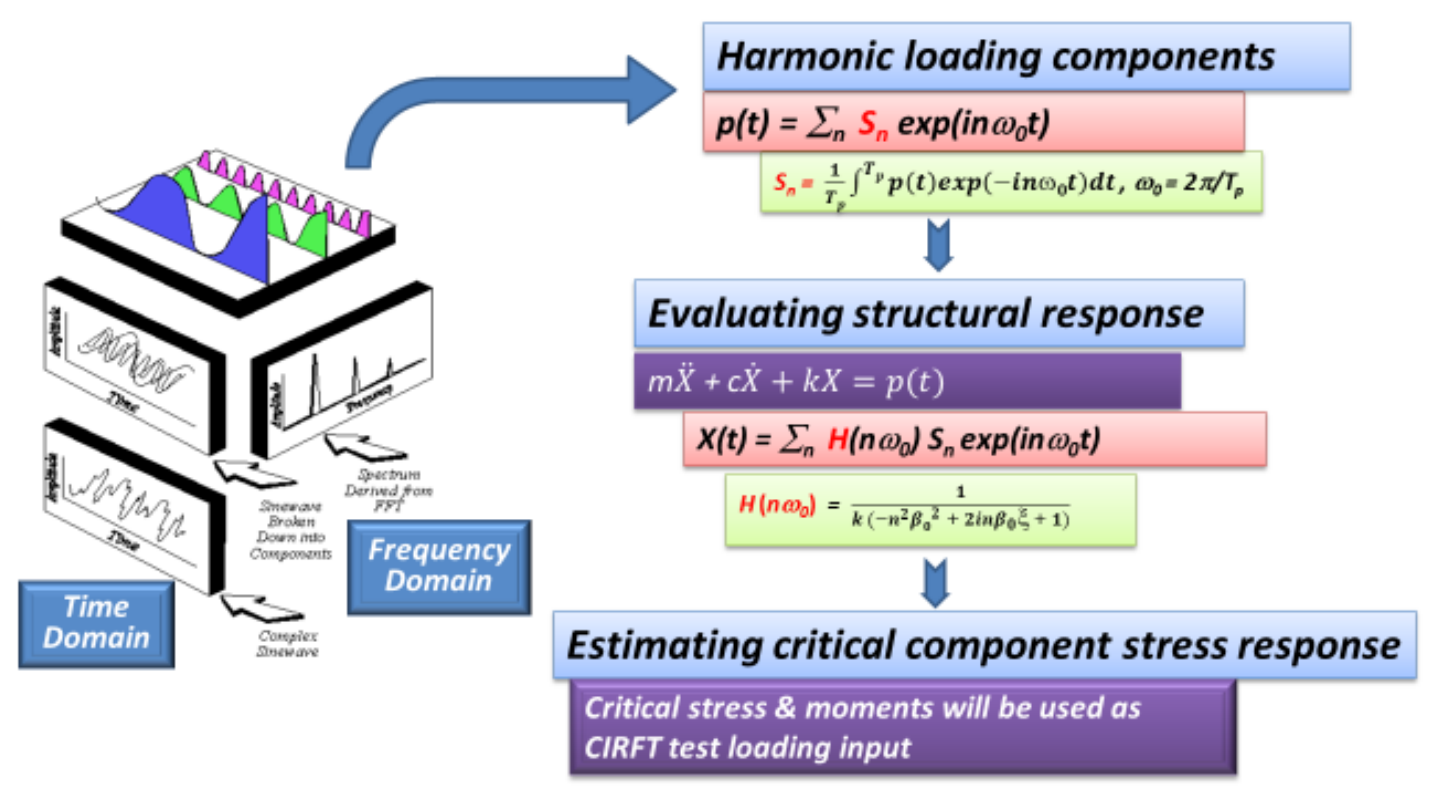

Fig. 13 The schematic diagram of conducting random-frequency response analyses. 


\subsection{Drop Impact Tester Design Configurations \& Development}

The schematic diagram of the first-generation drop impact tester is shown in Fig. 14.

The magnet driver is used as attaching and releasing projectile mechanism, in conjunction and synchronizing with the top-mounted stepper motor lifting mechanism automatically through in-house developed control systems during drop impact testing operation.

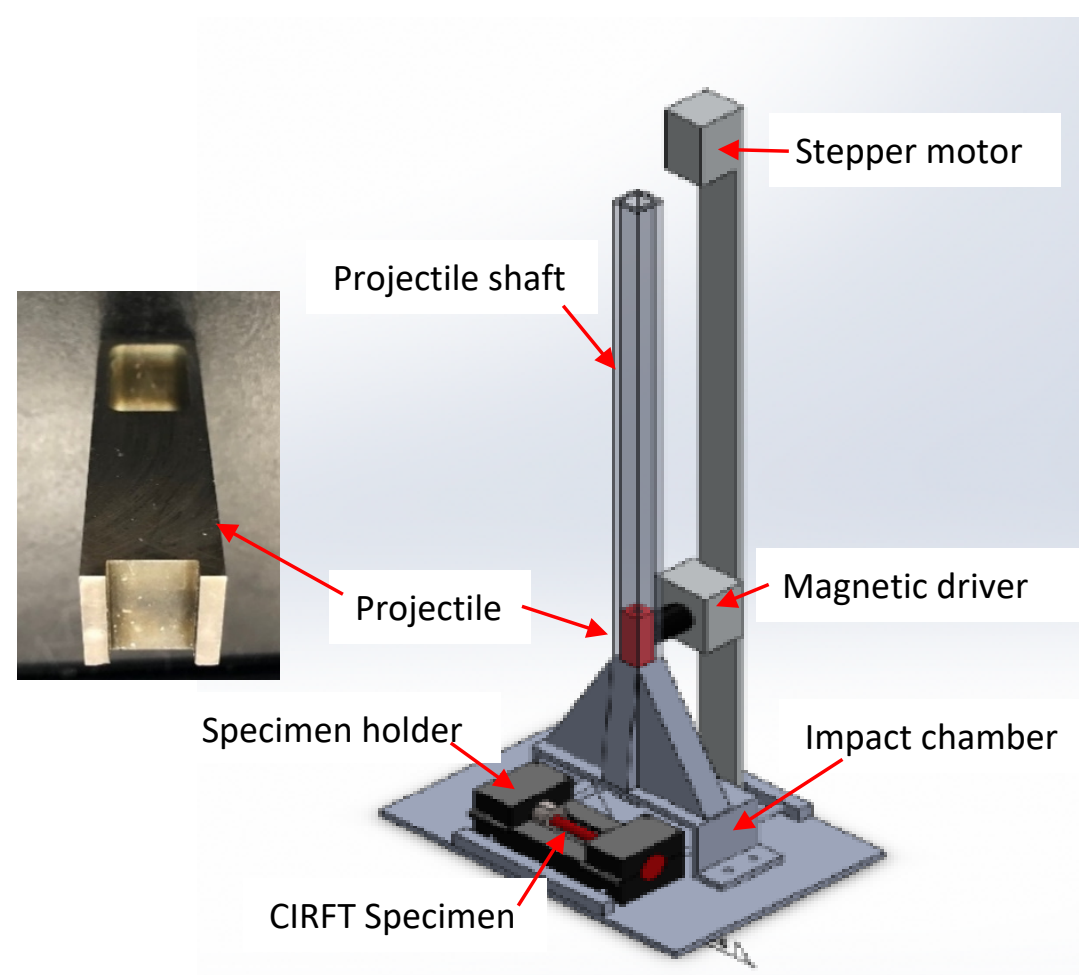

Fig. 14 Schematic diagram of drop impact tester design with a top-mounted stepper motor for lifting projectile.

The impact shaft and specimen chamber, including CIRFT specimen line up in specimen holder, are shown in Fig. 15 and Fig. 16. 

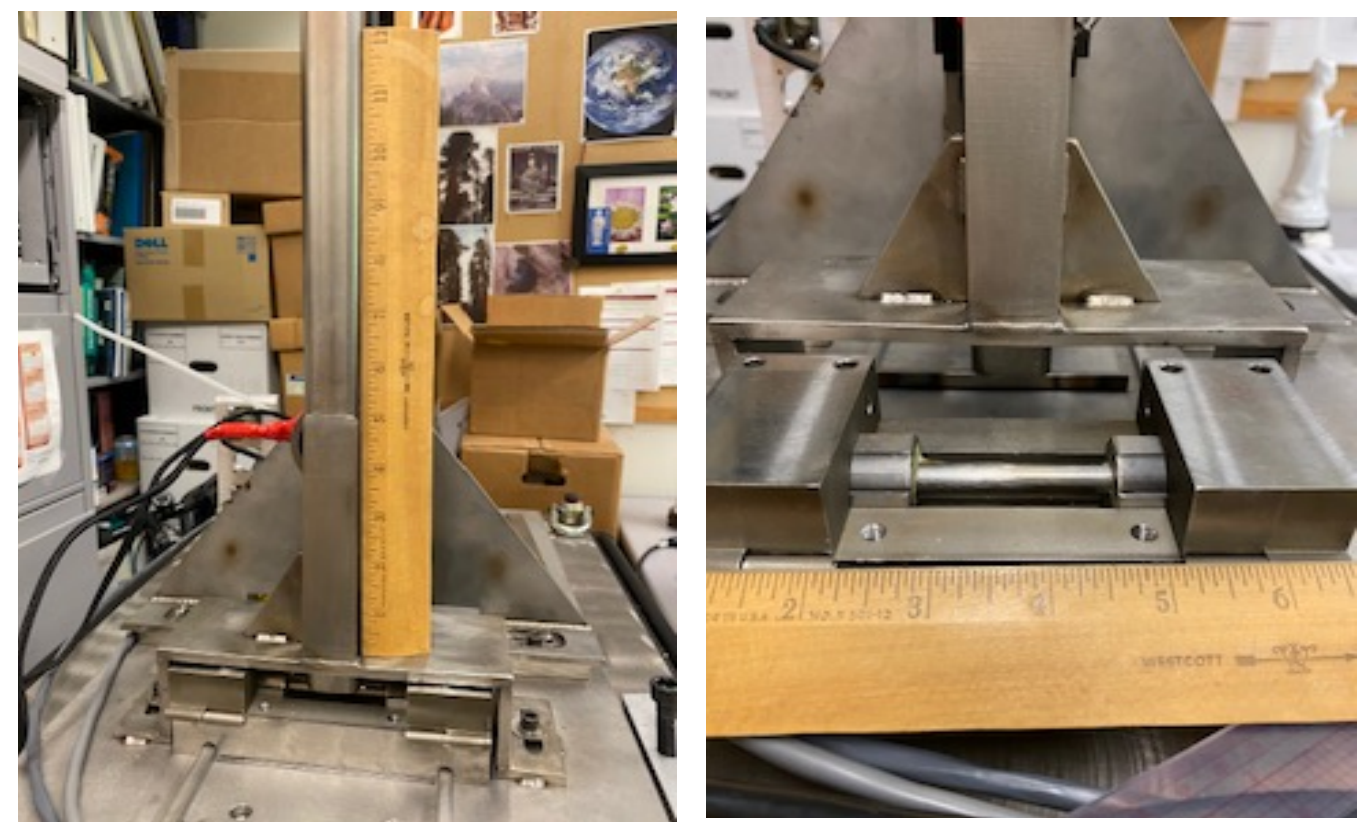

Fig. 15 (Left) impact shaft and specimen holder, (Right) is the close look of specimen holder pulled out of impact chamber. The clearance between impact projectile exit and the bottom the supporting plate is about 1-1/4". The clearance between specimen rod (2-inch gage section) and specimen holder is about 0.3 ".
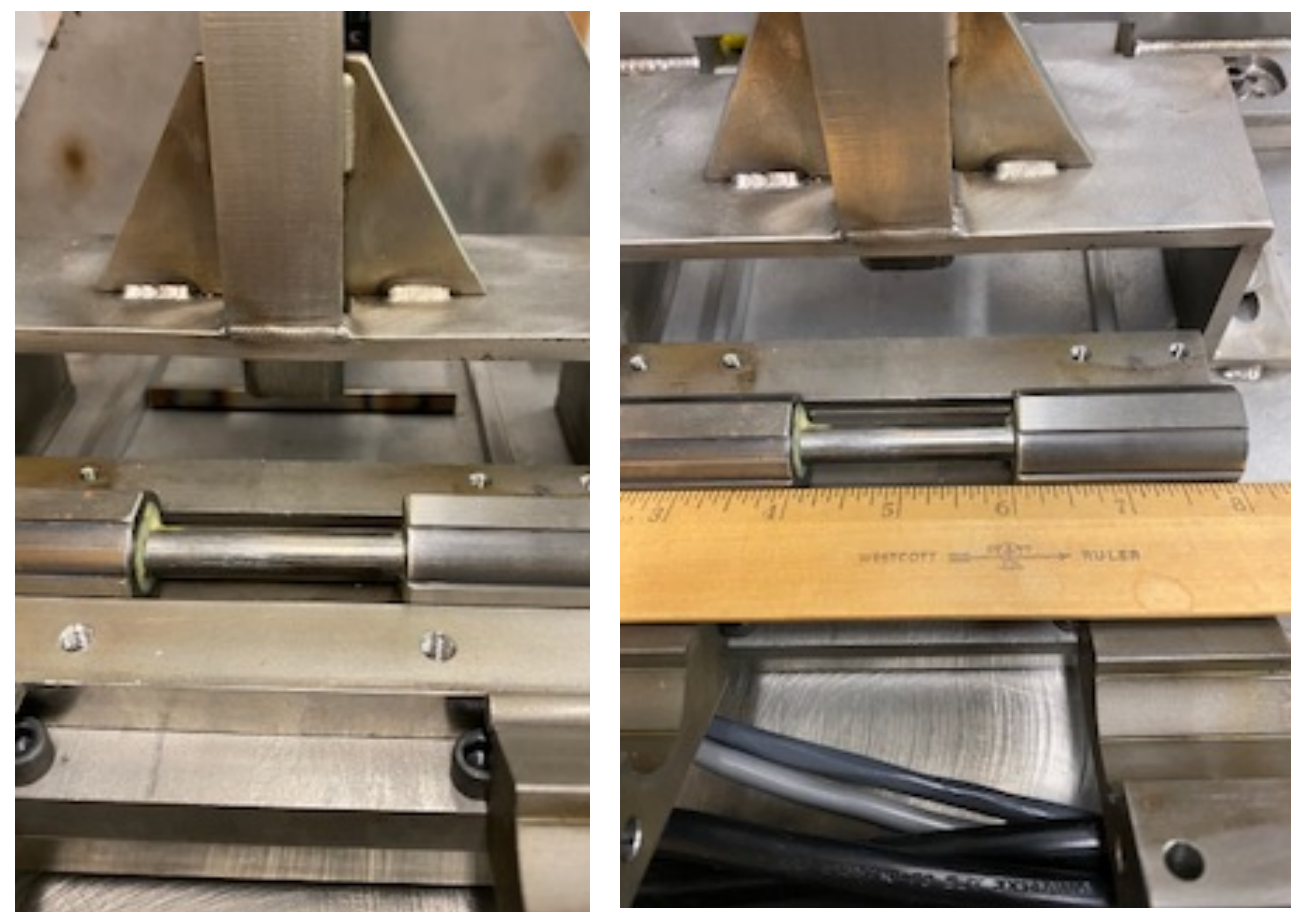

Fig. 16 Line-up view of CIRFT specimen and bottom of specimen holder. 
The lift stepper motor of the first-generation drop impact tester is aligned with the loading shaft and located on the top of the loading shaft (limit to only smaller size of stepper motor). In order to have a better structural stability and a smoother run with a heavy projectile, base-mounted large stepper motor is required and used in the second generation drop impact tester set-up, as shown in Fig. 17.
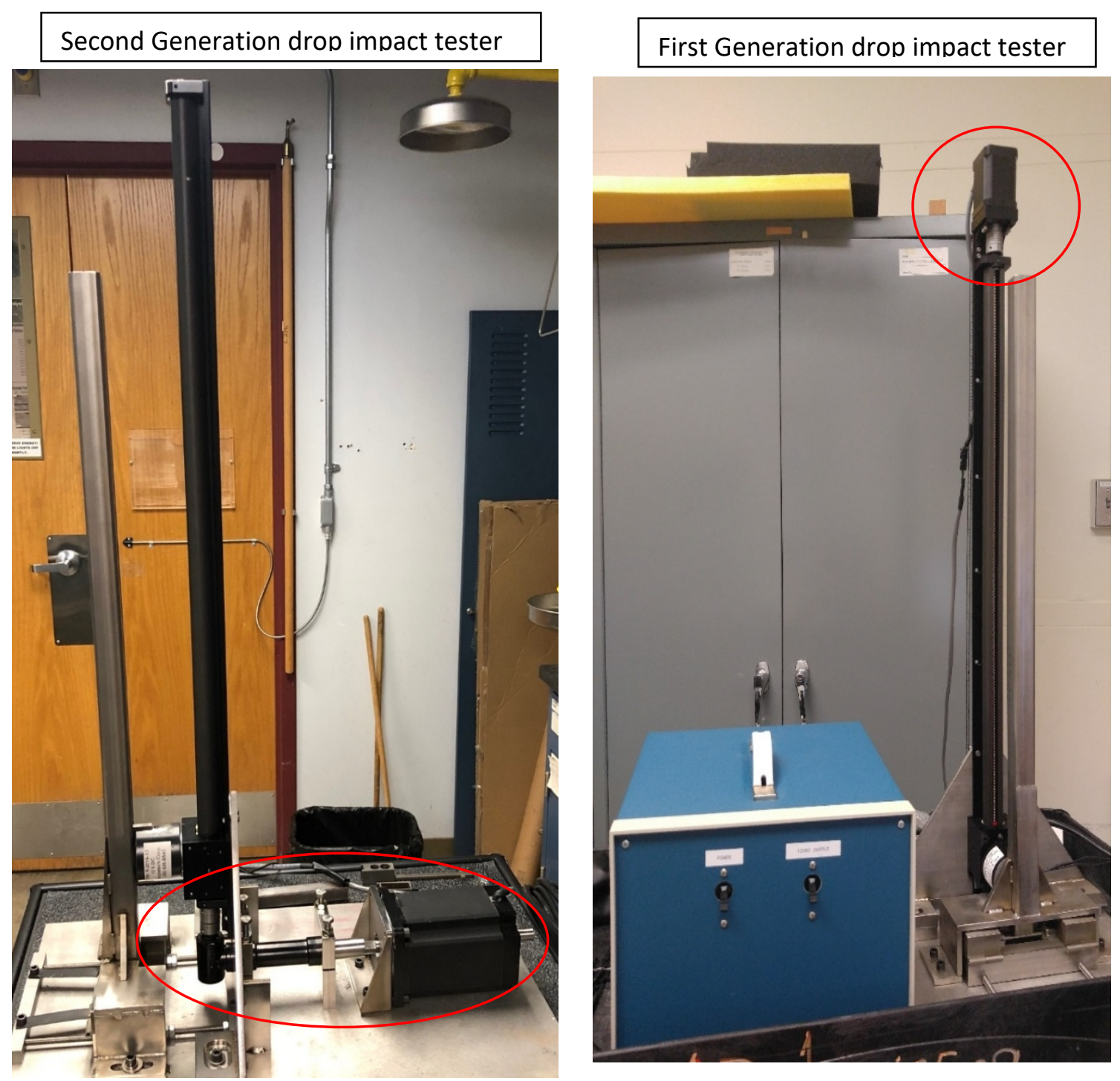

Fig. 17 (Left) The second generation drop impact tester, where the mechanical lifter is located on the bottom of the moving track; (Right) The first generation drop impact tester, where the mechanical lifter is located on the top of the moving track.

The second-generation loading train driver is located at the bottom plane of the loading shaft, which is in contrast to the first generation's loading train that is located at the top of the loading shaft, shown in Fig. 18. Additional fitting components were developed at the bottom loading shaft to change the loading force from horizontal set-up to a $90^{\circ}$ vertical shift orientation as shown in the Fig. 18. 


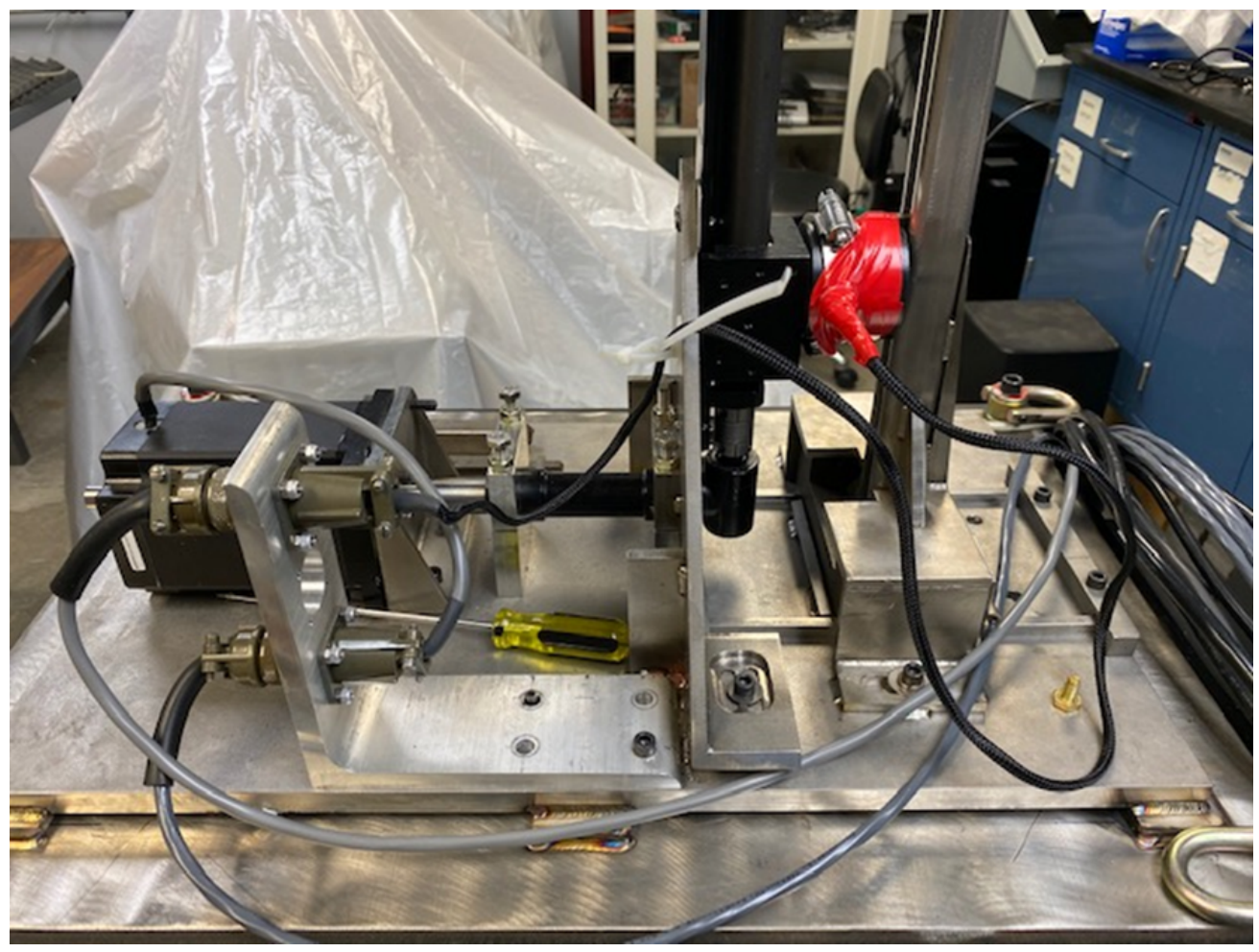

Fig. 18 Close view of the loading train for floor mounted driver, including an L-angle connector for magnet driver and stepper motor wrings for a hot-cell implementation.

The designed drop impact tester is fully automated without user intervention during impact testing to reach the targeted impact cycles.

The operation instruction manual of the developed Automated Drop Impact Tester is described below.

1. Start by making note of how large of a drop distance you require for the appropriate impact force

2. Using the formula 400 steps to 1 inch to convert the drop distance into steps

3. Turn on the main power supply as well as the $12 \mathrm{~V}$ power supply and the power for the PLC controller.

4. Connect the PLC controller to the PC using the attached CAT5 cable.

5. Once connection has been established, open the Do-More designer software and open the multicycle program.

6. On the program ladder input the appropriate number of steps in the axis control boxes; and enter the desired number of cycles in the stop control area.

7. Once programming is complete, check the program and download to the PLC controller.

8. Upon completion on the download, switch the PLC to the run position and the test will begin. 
9. Once the test is complete, switch the PLC to "STOP" and the to "TERM"

10. Open the PLC drop menu on the Do-More software and select "clear PLC memory"

11. In the memory clear menu box, uncheck the "load from PLC upon completion" box then finish the memory clearance.

12. Load Program "change sample" and download to PLC

13. With the PLC to "RUN" and wait for the weight to be held above the specimen.

14. Change out the specimen and wait for the weight to move back down on top of the specimen.

15. Switch the PLC back to "STOP" then "TERM".

16. Repeat steps 5-10 for new test.

Further modifications of the developed drop impact tester for hot-cell implementation were carried out, as shown in Fig. 19. 


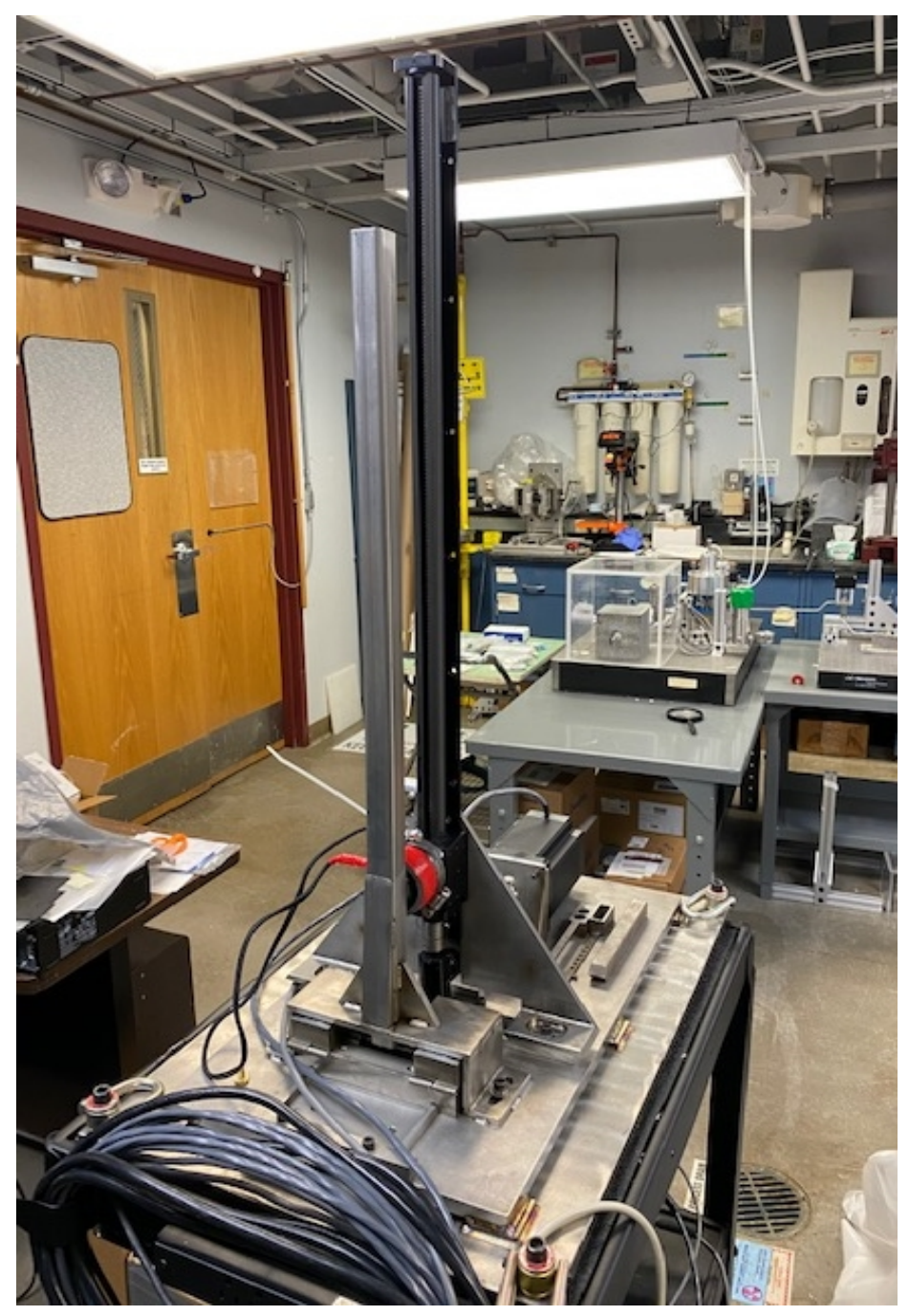

(a) 


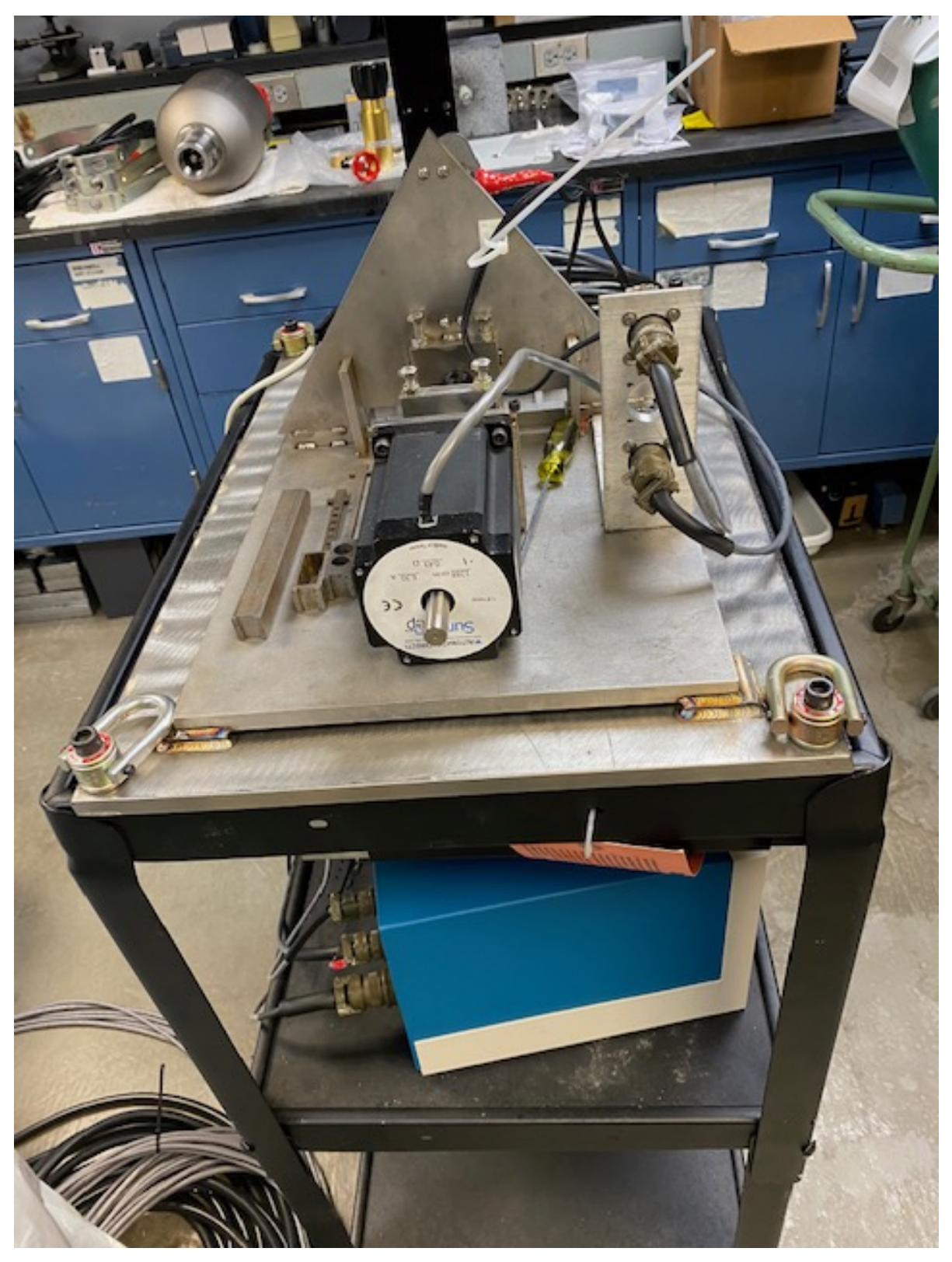

(b)

Fig. 19 Drop impact tester hot-cell implementation (a) wire length increased to 25 feet, and (b) lifting plate added with leveling functionality was added to the drop impact tester for hot-cell installation. 


\section{REFERENCE}

1. Wang, Jy-An, Jiang, Hao, Wang, Hong. Dynamic Deformation Simulation of Spent Nuclear Fuel Assembly and CIRFT Deformation Sensor Stability Investigation. ORNL/SPR-2015/662. Oak Ridge, TN: Oak Ridge National Laboratory.2015.

2. Jiang, Hao, Wang, Jy-An. Spent Nuclear Fuel System Dynamic Stability under Normal Conditions of Transport, Journal of Nuclear Engineering and Design, 310 (2016) 1-14, October 6, 2016.

3. McConnell, P., Wauneka, R., Saltzstein, S., Sorenson, K., 2014. US Department of Energy Used Fuel Disposition Campaign, Normal Conditions of Transport Truck Test of a Surrogate Fuel Assembly. Sandia National Laboratories. FCRD-UFD-2014-000066.

4. Wang, Jy-An, Wang, Hong, Jiang, Hao, Bevard, Bruce. High burn-up spent nuclear fuel transport reliability investigation, Nuclear Engineering and Design Journal, 330 (2018) 497-515, April 2018. 


\section{APPENDIX A: DYNAMIC DEFORMATION SIMULATIONS OF SPENT NUCLEAR FUEL ASSEMBLY [1]}

\section{A1. INTRODUCTION}

This research supports the US Department of Energy (DOE) Used Fuel Disposition Campaign modeling, simulation, and experimental integration research, development, and deployment (RD\&D) plan for spent nuclear fuel (SNF) integrity and structural performance under normal conditions of transportation (NCT) [1]. The PWR fuel assembly skeleton, as shown in Fig. 20, is formed by guide tubes and spacer grids designed to constrain fuel rods during a reactor operation. In a vertical setup, the skeleton was subjected to vibration loads induced by fluid dynamics, and the rods' dead weight was transmitted though the spacer grids to the guide tubes during reactor operation. When the SNF assembly is in a horizontal layout under NCT, the skeleton formed by the guide tubes and spacer grids becomes the primary load-bearing system that carries and transfers the vibration loads within an SNF assembly. This includes interaction of forces between the SNF assembly and the canister basket walls. Therefore, the integrity of guide tubes and spacer grids will critically affect the vibration intensity of the fuel assembly during transport and will consequently affect the multipurpose purpose canister (MPC) design concept development to ensure safe SNF transport.

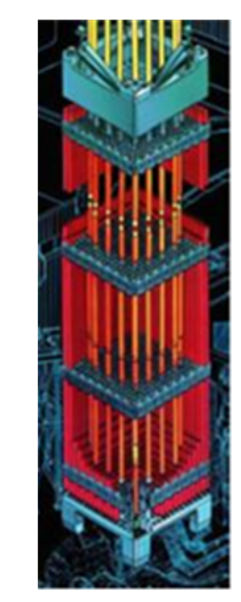

Fuel assembly skeleton

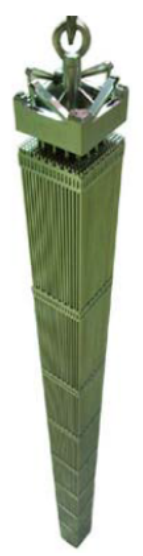

Fuel assembly

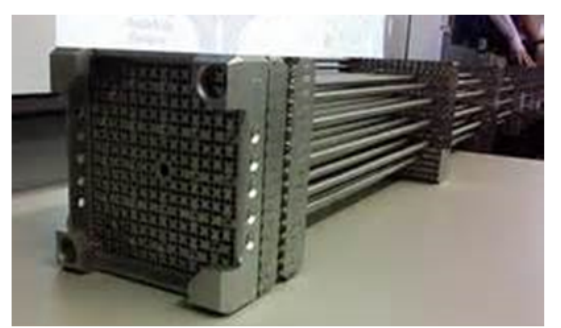

Fuel skeleton horizontal setup

Fig. 20. SNF assembly schematic.

Random vibration registered at the SNF transport cask, which is excited from the railcar bed, provides the external loading driver to vibrate the SNF assembly. In addition to this external vibration driver, the fuel assembly also registers internal transient shocks resulting from the dynamic interactions among the fuel assembly components inside the cask. These components include the skeleton, fuel rods, and canister basket walls. Their dynamic interactions can significantly increase the high-rate impact loading intensity within fuel assembly components during NCT. 
The aging or fatigued skeleton system can increase contact impact loading intensity between fuel rods, and it can enhance the probability of SNF resonance vibration deformation. The contact interactions between fuel rods, spacer grids, and the basket wall can also increase fuel rod transient shock loads frequencies. Thus external cask vibration needs to be mitigated, along with internal amplification from the fuel assembly system vibration and its interaction transient shocks. System damping may need to be increased to reduce vibration intensity. Such mitigations will eventually impact the canister system design.

This research investigates the SNF assembly deformation dynamics during normal vibration mode, as well as the cask's internal transient shock mode during NCT. The finite element method (FEM) was used to develop vibration simulation modeling for the targeted SNF subassembly. Dynamics analyses were performed in the frequency domain to study frequency characteristics of the fuel assembly system and in the time domain to simulate the transient dynamic response of the fuel assembly. Finite element analysis (FEA) simulation results are used to determine the stress and strain states of submodel components, such as fuel rods, the guide tube, spacer grids, nozzles, and the basket wall under vibration loading during normal vibration condition and transient shocks during NCT. The resulting stress/strain data can be used in further fuel assembly component failure analyses. The contact interactions between system components during transient dynamic simulation have also been investigated. 


\section{A2. SNF ASSEMBLY DYNAMIC SIMULATION}

The FEA modeling effort was focused on the SNF assembly in a transportation mode in a horizontal setup. Fig. 21 shows a fuel assembly consisting of fuel rods, the bottom nozzle, the top nozzle, guide tubes, and spacer grids.

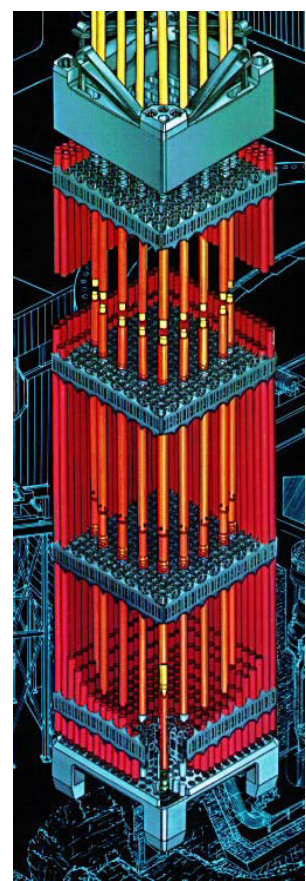

(a) Fuel assembly skeleton

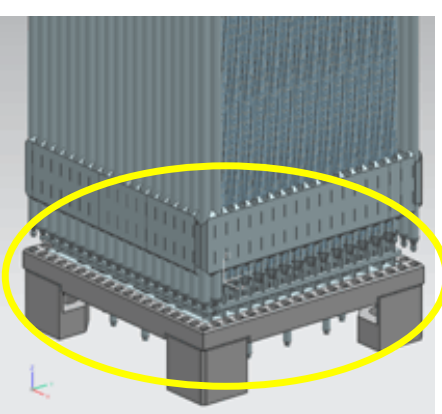

(b) Bottom nozzle

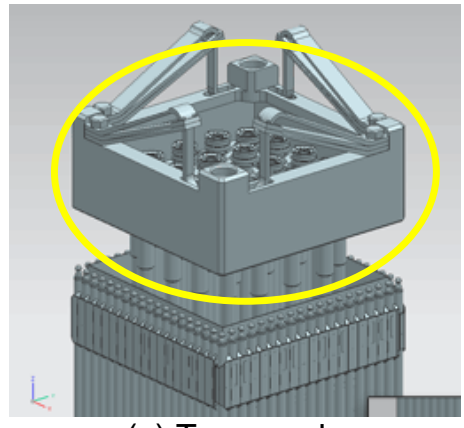

(c) Top nozzle

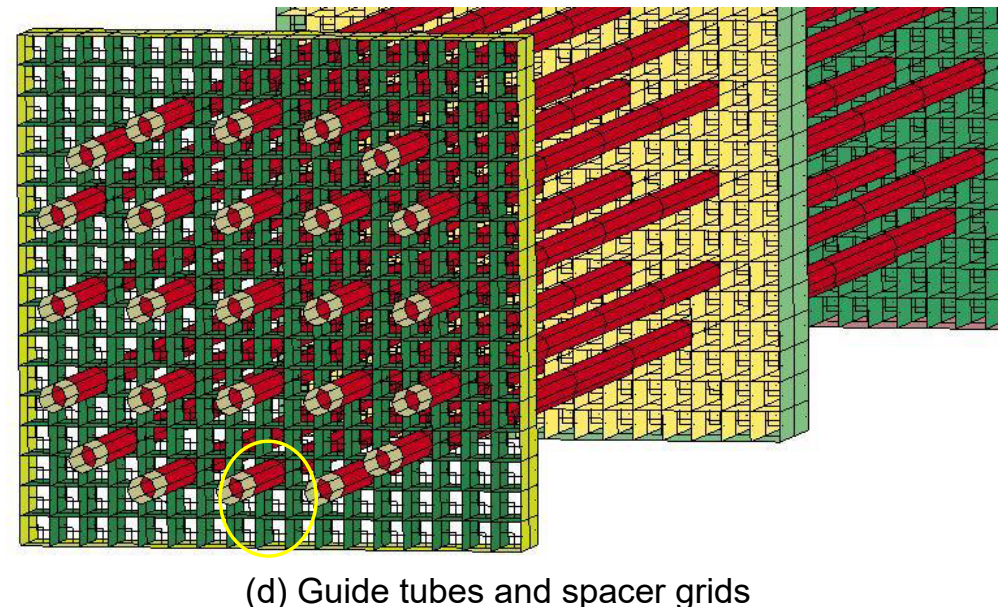

Fig. 21. $17 \times 17$ PWR fuel assembly.

The targeted SNF assembly model is a three dimensional ABAQUS-explicit transient FEM of a simplified single fuel assembly. The fuel rod in the simplified geometry is 144 inches long. To simulate the contacts of fuel pellets, 240 0.6-inch fuel pellets are used in the model. Fig. 22 shows the modeled 240 fuel pellets in a 144-inch long fuel rod, where pellets contact each other at the ends. In one fuel rod, there are 239 contact pairs within the fuel pellets. In order to have a meaningful, practical investigation of the SNF rod's dynamic response and the SNF system components' interactions during NCT, the SNF assembly was simplified as a horizontally oriented submodel (Fig. 23). The skeleton of the SNF assembly consists of guide tubes and spacers, which are the primary structural components carrying the vibration loading excited from the railcar bed and transferred from outside the cask. Below are the key assumptions and primary simplifications made in the targeted submodel development.

- Two fuel rods with a guide tube as highlighted in Fig. 21d at the bottom edge of full fuel assembly are modeled. The guide tube is on top of two fuel rods, as illustrated in Fig. 23. Each fuel rod has 240 fuel pellets and a 144 -inch long cladding. 
- The guide tube and two fuel rods are connected with eight spacers, including top and bottom Inconel spacers, as well as six intermediate Zr-4 spacers (Fig. 23 and Fig. 24).

- The spacer grids are modeled as simplified box structures, and the spacer grid leaf springs/dimples are modeled as translational springs.

- Fuel rods are connected to spacer grids through the springs/dimples, as shown in Fig. 25.

- The guide tube is rigidly connected to the spacer grids via the tie constraint in ABAQUS.

- The top and bottom nozzles are simplified as two plates to eliminate unnecessary geometric details.

- A long rectangular plate is modeled to represent the basket floor and is assumed as part of the basket enclosure to interact with the fuel rod assembly from the bottom, as shown in Fig. 23

- The guide tube is connected to the nozzles via the tie, and the nozzles are tied to the basket to properly transfer the dynamic loading, as shown in Fig. 26.

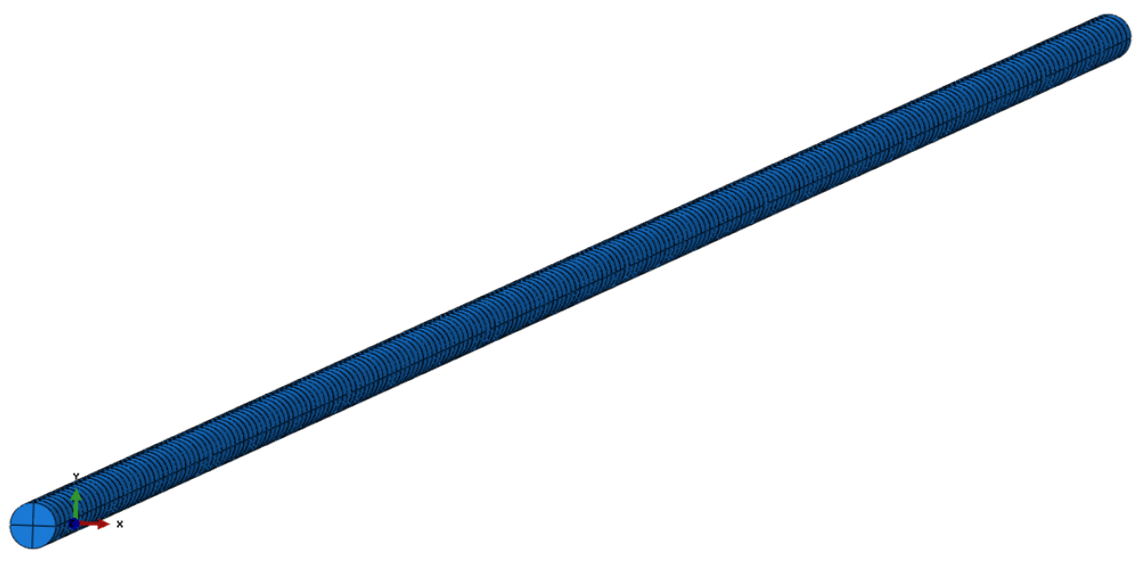

Fig. 22. The modeled 240 fuel pellets in one fuel rod.

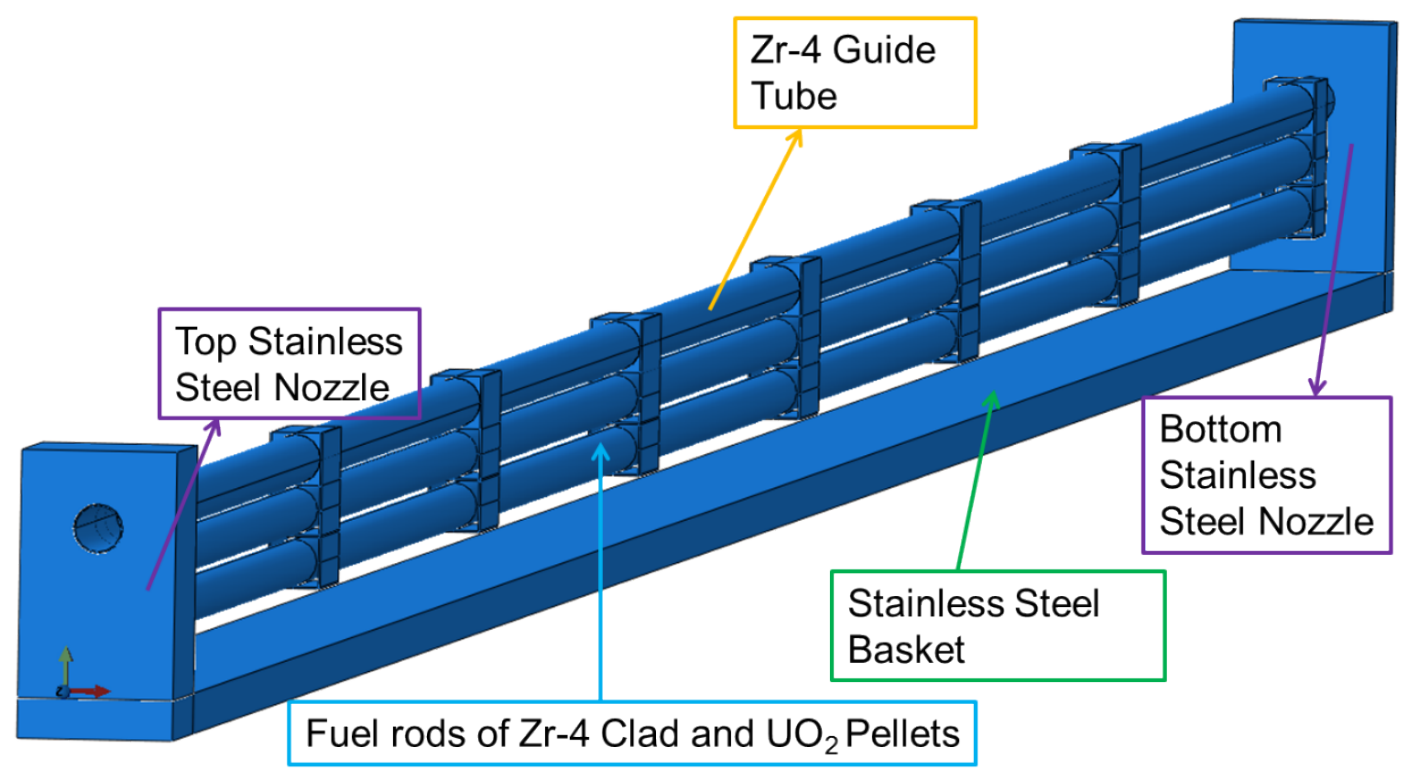


Fig. 23. SNF assembly submodel for normal transportation evaluation.

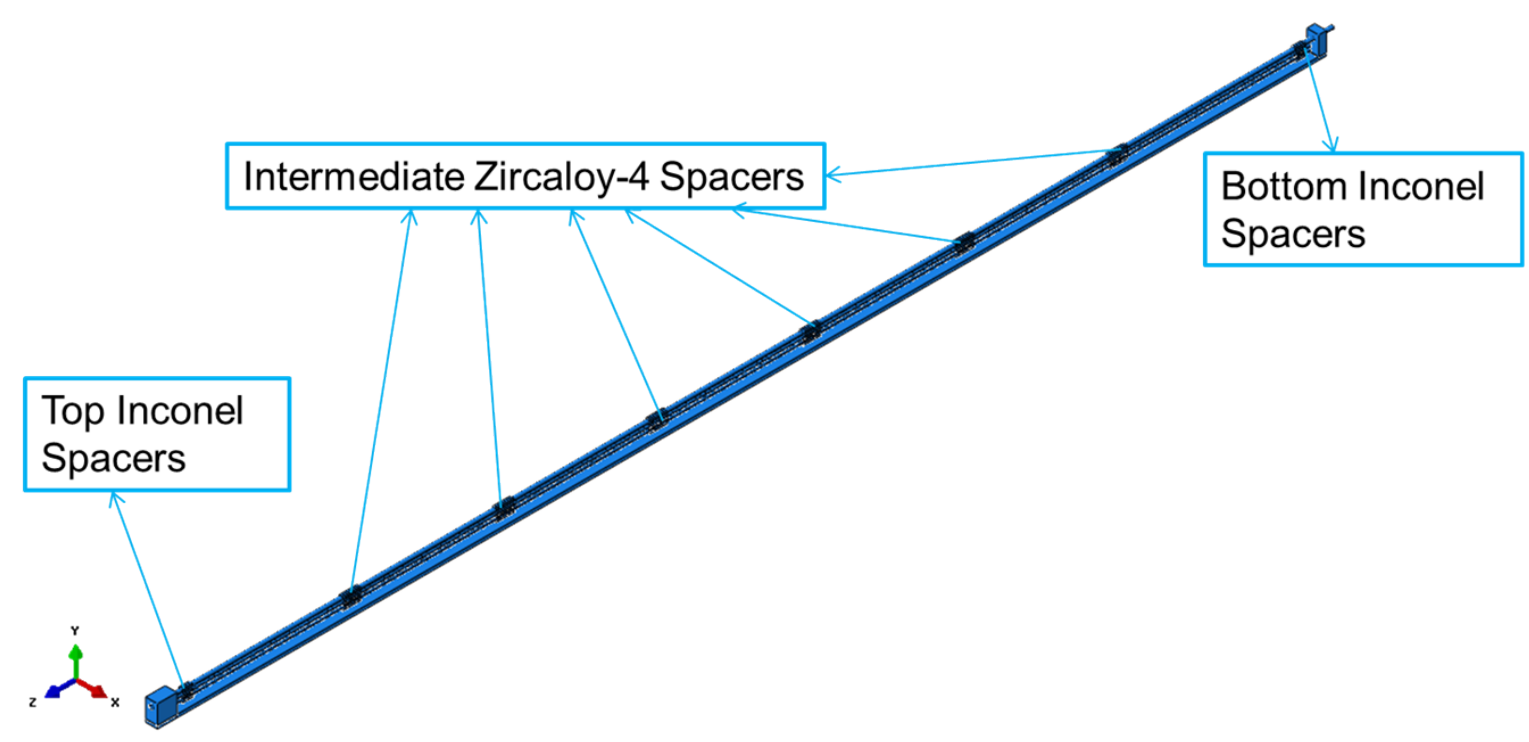

Fig. 24. SNF assembly submodel with spacers.

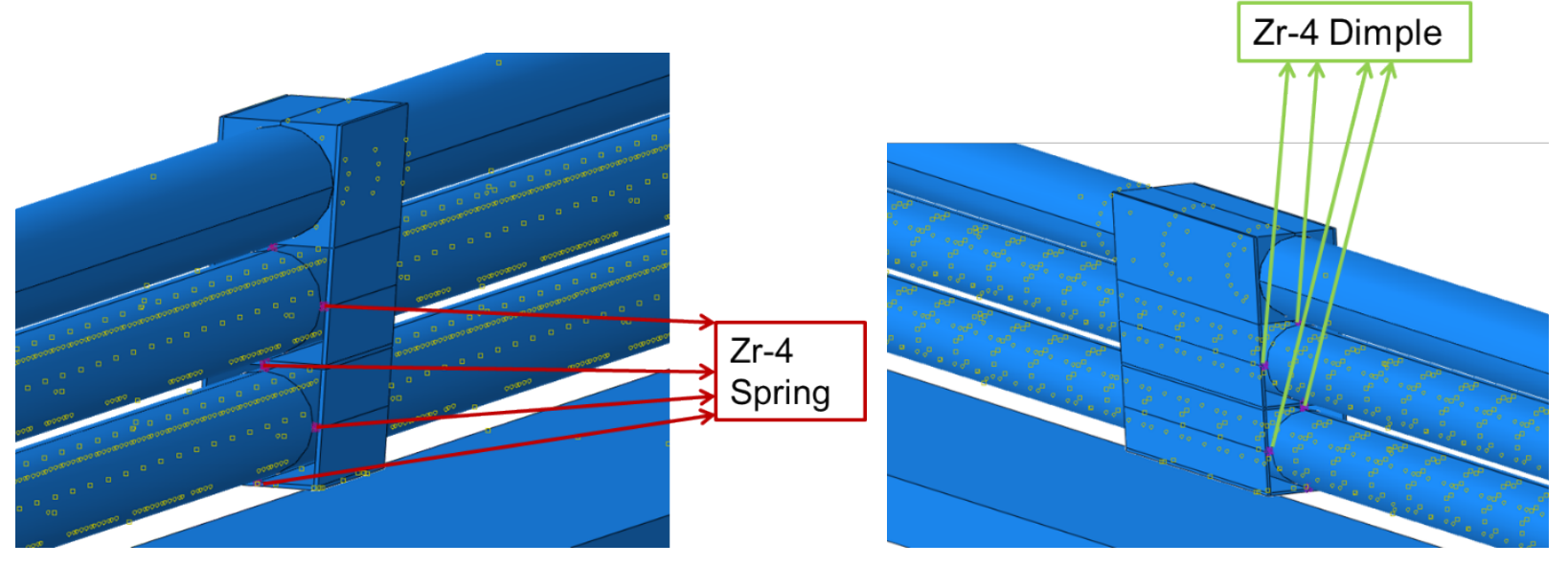

Fig. 25. Spacer grid leaf spring/dimples modeled as translational springs.

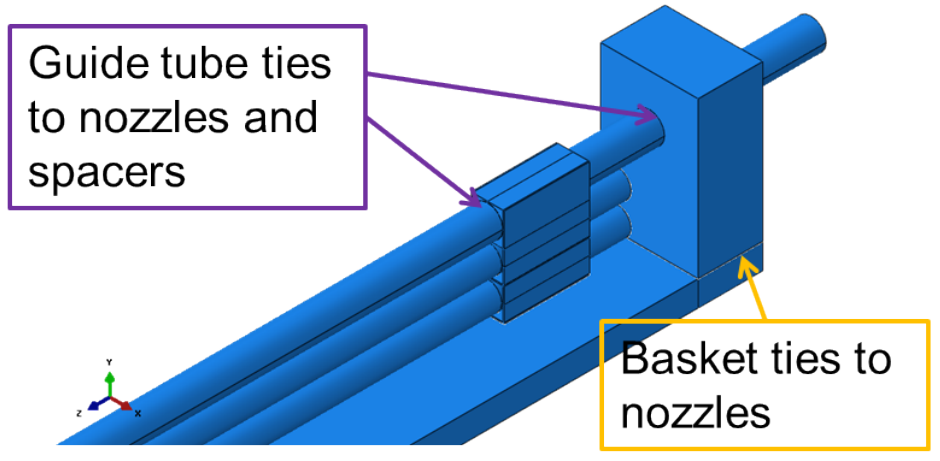

Fig. 26. Tie constraint to transfer dynamic loading. 
The geometry of the fuel assembly submodel is taken primarily from dimensions provided in DOE's Used Nuclear Fuel Loading and Structural Performance Under Normal Conditions of Transport-

Demonstration of Approach and Results of Used Fuel Performance Characterization [2] and summarized in Table 1. This fuel assembly submodel retains the proper mass and inertial properties for dynamic simulation.

Table 1. Dimensions for fuel rods and fuel assembly

\begin{tabular}{lc}
\hline Dimension & Value (inch) \\
\hline Fuel pellet length & 0.6 \\
Fuel pellet diameter & 0.382 \\
Fuel cladding length & 144 \\
Fuel cladding outer diameter (OD) & 0.36 \\
Guide tube OD & 0.474 \\
Guide tube inner diameter (ID) & 0.45 \\
Guide tube length & 150 \\
Intermediate spacer axial length & 2.25 \\
Intermediate spacer shell thickness & 0.018 \\
Intermediate spacer width & 0.51 \\
Top and bottom spacer axial length & 1.322 \\
Top and bottom spacer shell thickness & 0.018 \\
Top and bottom spacer width & 0.51 \\
Bottom nozzle plate thickness & 1 \\
Bottom nozzle width & 1.51 \\
Bottom nozzle height & 2.4077 \\
Top nozzle plate thickness & 2.5 \\
Top nozzle width & 1.51 \\
Top nozzle height & 2.4077 \\
Basket floor plate thickness & 0.3937 \\
Basket floor plate length & 148.5 \\
Basket floor plate width & 1.51 \\
\hline Distance form base of bottom nozzle to: & \\
\hline Top of $1^{\text {st }}$ spacer grid & 3.5 \\
Top of $2^{\text {nd }}$ spacer grid & 27.08 \\
Top of $3^{\text {rd }}$ spacer grid & 45.66 \\
Top of $4^{\text {th }}$ spacer grid & 66.02 \\
Top of $5^{\text {th }}$ spacer grid & 85.49 \\
Top of $6^{\text {th }}$ spacer grid & 104.96 \\
Top of $7^{\text {th }}$ spacer grid & 124.43 \\
Top of $8^{\text {th }}$ spacer grid & 144.82 \\
\hline
\end{tabular}

Fig. 27 shows the mesh of the SNF fuel assembly submodel. All components are meshed with 8-node linear hexahedral brick elements. The mesh is coarsened to optimize the solution time while not losing accuracy. The fuel rods are supported by two sets of translational springs in every slot: one set of leaf springs and one set of dimples. ABAQUS explicitly requires that the incremental time between the calculation steps be smaller than the shortest duration of stress wave propagation through an element. This is accomplished by following Courant-Friedrichs-Levy stability criterion. Special care is taken to stabilize the dynamic calculation, including mesh distortion control, severe distorted element deletion, reduced integration and hourglass control, etc. However, a long solution time for dynamic simulation is 
still anticipated since the very small time increment on the order of $1.0 \mathrm{E}-8 \mathrm{~s}$ is required to converge the solution. Furthermore, the model consists of roughly 130,000 nodes and 80,000 elements, which also result in a long solution time.

Contact is implemented throughout the fuel assembly between interacting components. The most time consuming and tedious work is to define the 239 surface-to-surface contact pairs between the 240 fuel pellets. Surface-to surface contacts between the guide tube and fuel rod, between spacer grids and basket floor, and between fuel rod and basket floor are defined when they come to touch each other. As mentioned, the guide tube is rigidly attached to spacer grids and nozzles with tie constraints, and the fuel rods are supported by translational springs on the spacer grids. The friction coefficient is reasonably assumed as 0.3 at contact surfaces.

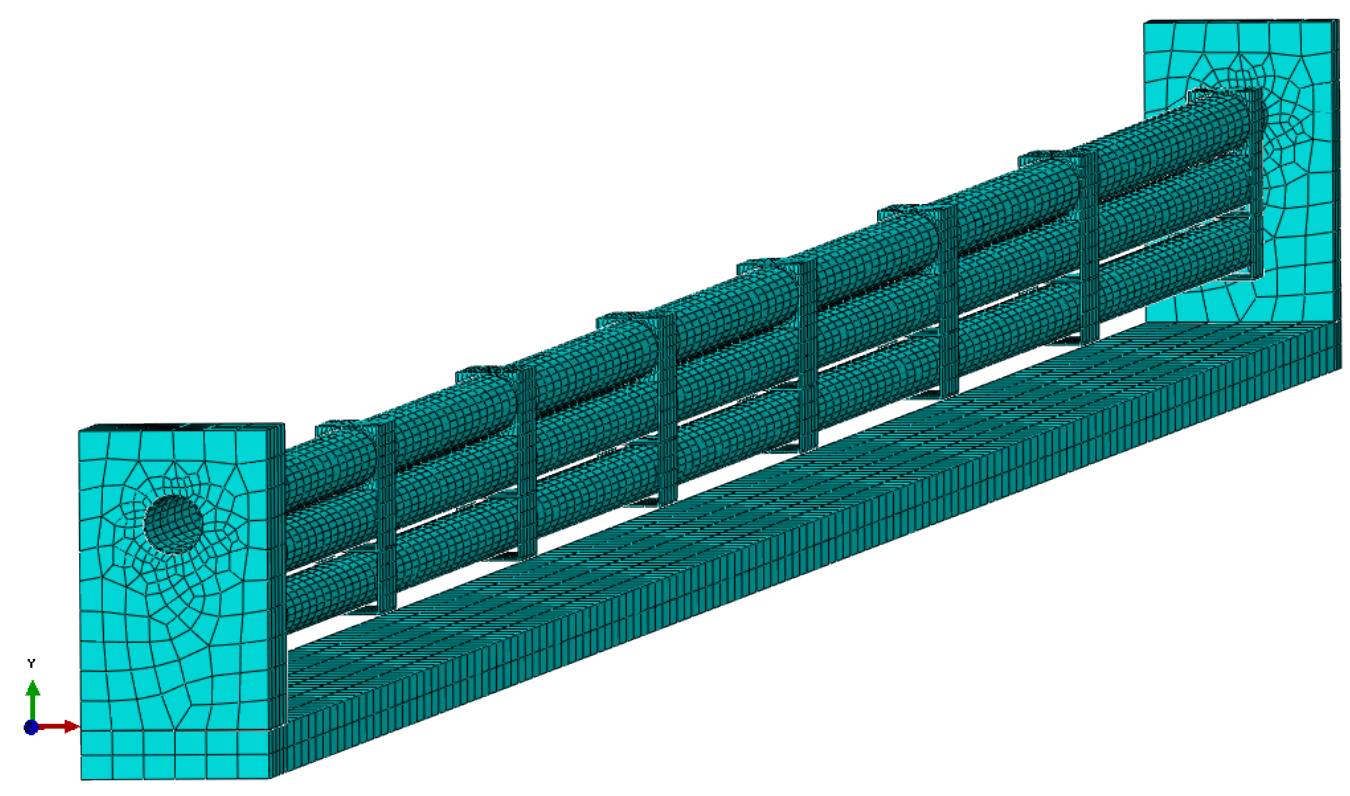

Fig. 27. Mesh of the fuel assembly submodel

As shown in Fig. 23 and Fig. 24, fuel pellets are made of $\mathrm{UO}_{2}$, while fuel cladding, the intermediate spacer grids, and the guide tube are made of Zircaloy-4. The top and bottom spacer grids are made of Inconel, and the top and bottom nozzles and basket are made of stainless steel. The material properties of the fuel assembly's components are listed in Table 2. The leaf spring and dimple spring material is Zircaloy-4. The spring stiffness value for Zircaloy-4 leaf spring is $280 \mathrm{lbf} / \mathrm{in}$, and the value for Zircaloy-4 dimple is $719.5 \mathrm{lbf} /$ in Ref. [2].

Table 2. Mechanical properties of the fuel assembly

\begin{tabular}{lcccc}
\hline Material & Young's modulus & Poisson's & Yield strength & Density \\
$(p s i)$ & ratio & $(\mathrm{psi})$ & $\left(\mathrm{lb} / \mathrm{in}^{3}\right)$ & \\
\end{tabular}




\begin{tabular}{llllll}
\hline Pellet & $\mathrm{UO}_{2}[3]$ & $2.92 \mathrm{e} 7$ & 0.32 & $3.11 \mathrm{e} 5$ & 0.396 \\
$\begin{array}{l}\text { Clad, } \\
\text { intermediate } \\
\text { spacer grids, } \\
\text { guide tube }\end{array}$ & Zircaloy-4 [3] & $1.32 \mathrm{e} 7$ & 0.33 & $1.31 \mathrm{e} 5$ & 0.237 \\
$\begin{array}{l}\text { Top and bottom } \\
\text { spacer grids }\end{array}$ & Inconel [4] & $3.06 \mathrm{e} 7$ & 0.284 & $1.432 \mathrm{e} 5$ & 0.296 \\
$\begin{array}{l}\text { Basket, nozzles } \\
\text { Stainless steel [3] }\end{array}$ & $2.8 \mathrm{e} 7$ & 0.3 & & \\
\hline
\end{tabular}

\section{A2.1 Modal Analysis}

Dynamics analysis of a vibrating system is often performed in the frequency domain because the dynamic response of structures depends on the frequency characteristic of the system, namely natural frequencies and mode shapes. When the excitation frequency loaded on the structure is near its natural frequency, resonance occurs, which will significantly amplify the system dynamic response to the extent that causes structure failure. Modal analysis is used to determine the natural frequencies of the SNF assembly submodel and the dominant modes. The results will provide a better understanding of frequency characteristic of the fuel assembly systems.

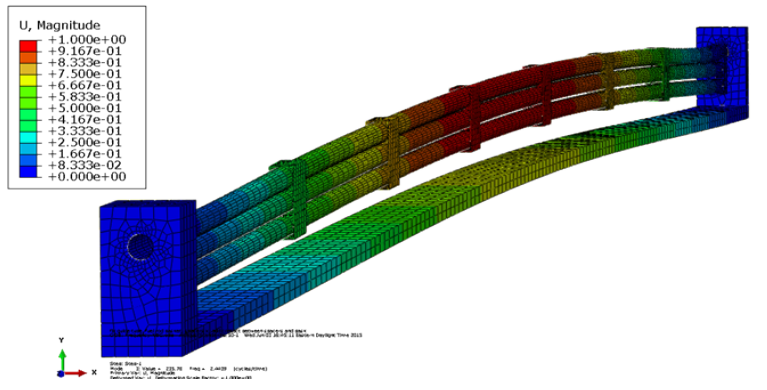

Mode-1: $2.444 \mathrm{~Hz}$

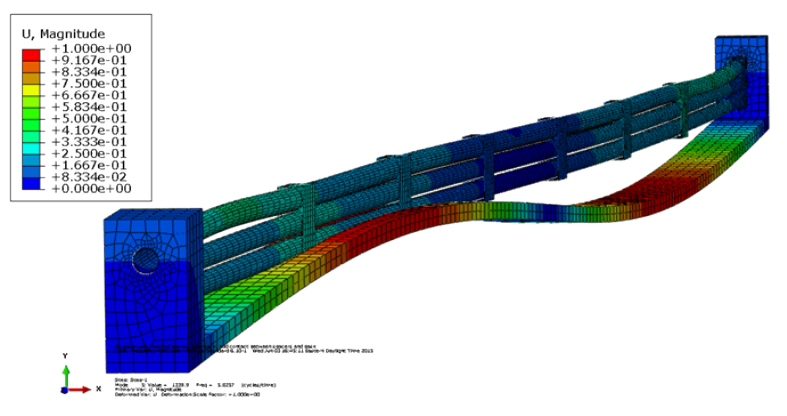

Mode-3: $5.826 \mathrm{~Hz}$

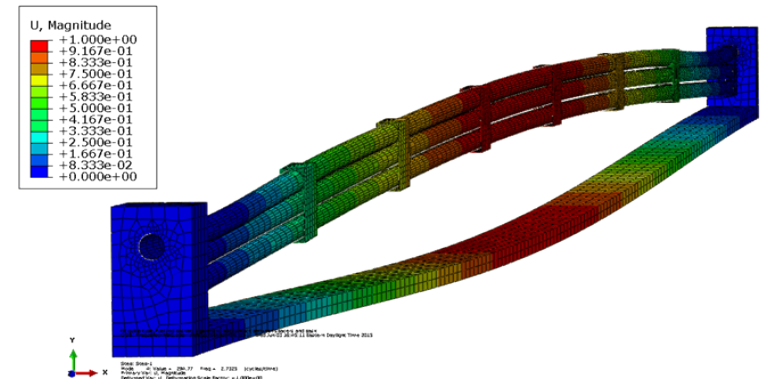

Mode-2: $2.733 \mathrm{~Hz}$

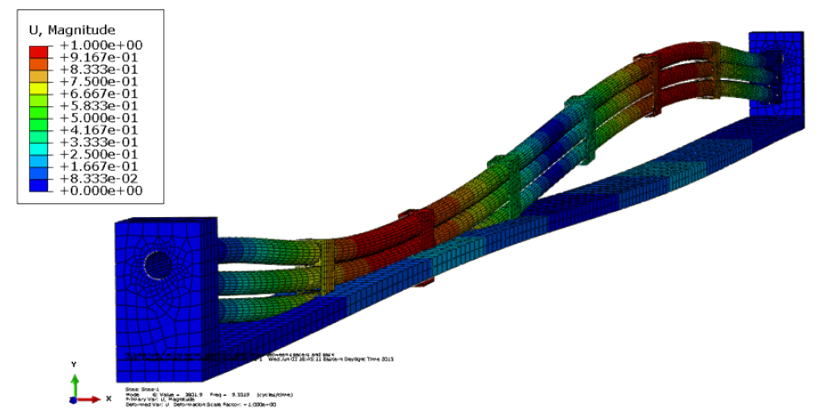

Mode-4: $9.552 \mathrm{~Hz}$ 


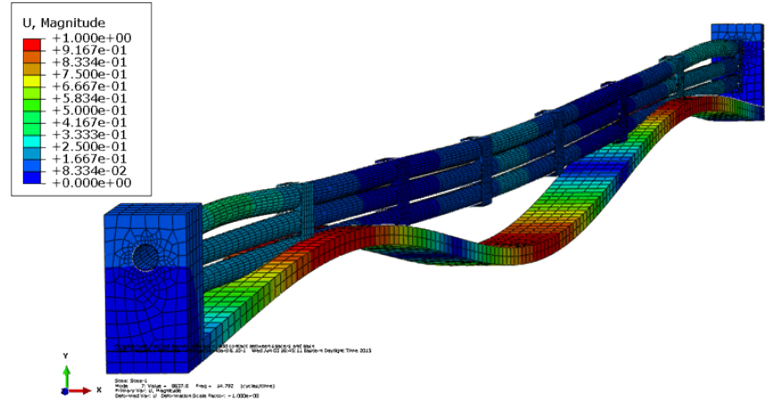

Mode-5: $14.792 \mathrm{~Hz}$

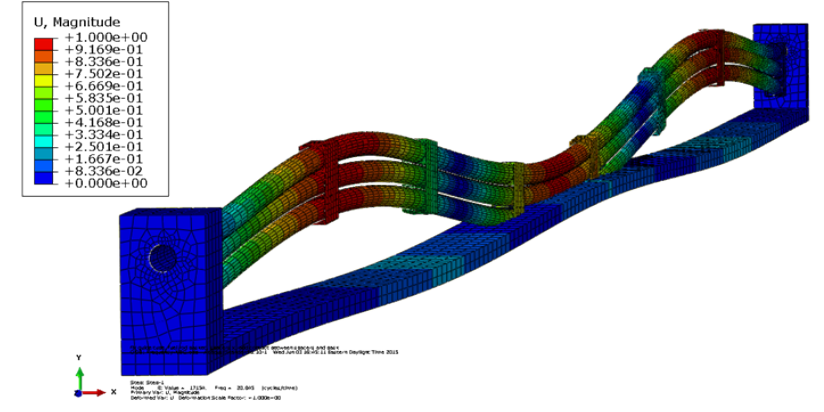

Mode-6: $20.845 \mathrm{~Hz}$

Fig. 28a. The first 6 modes and the associated natural frequencies of the fuel assembly submodel.

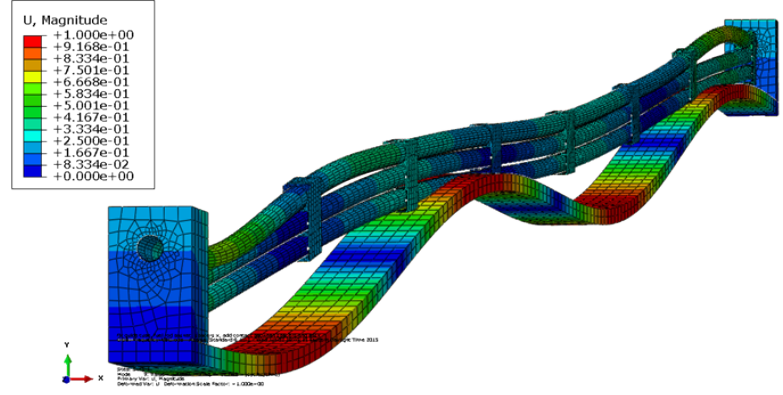

Mode-7: $22.665 \mathrm{~Hz}$

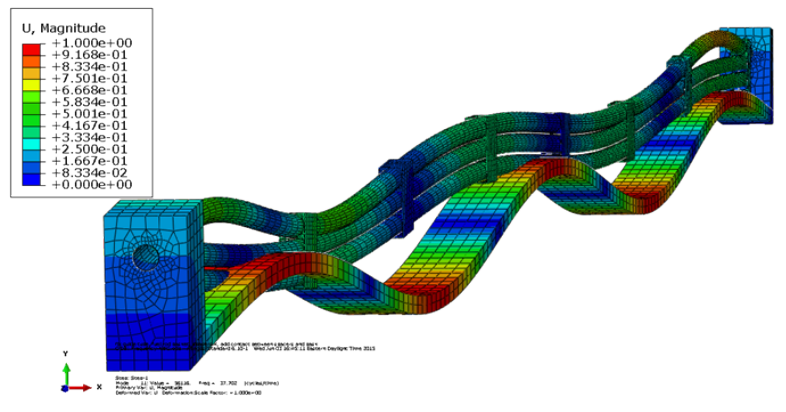

Mode-9: $37.702 \mathrm{~Hz}$

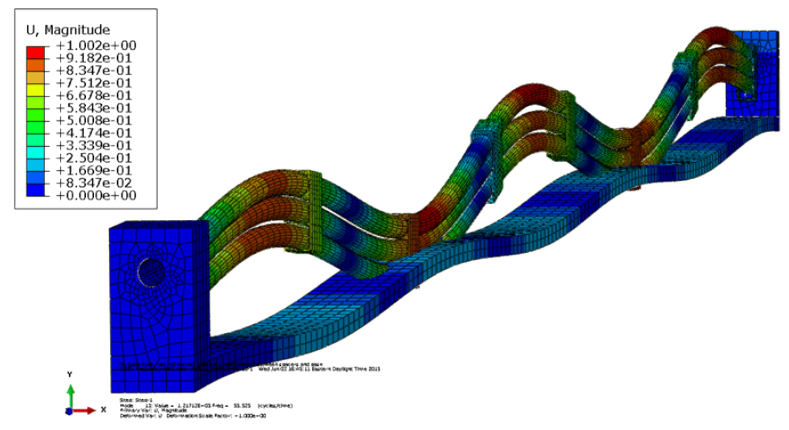

Mode-11: $55.525 \mathrm{~Hz}$

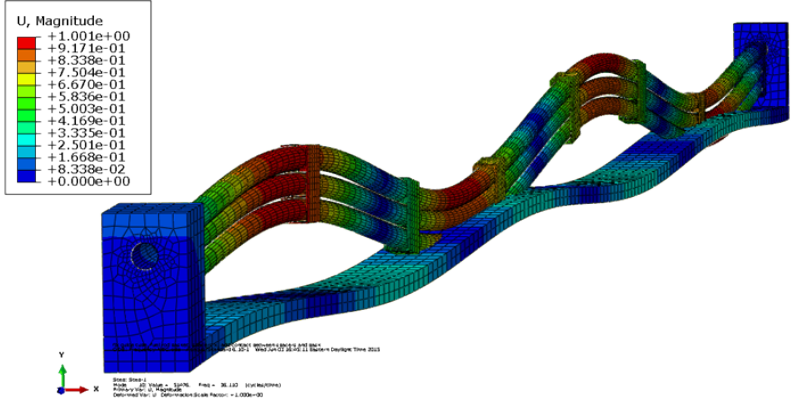

Mode-8: $36.11 \mathrm{~Hz}$

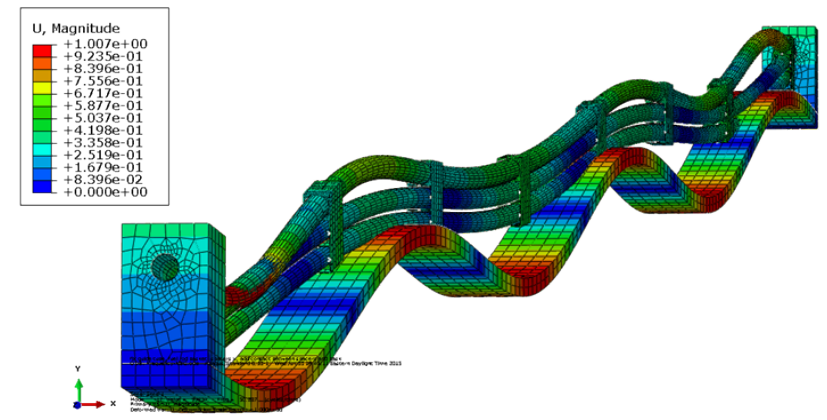

Mode-10: $50.287 \mathrm{~Hz}$

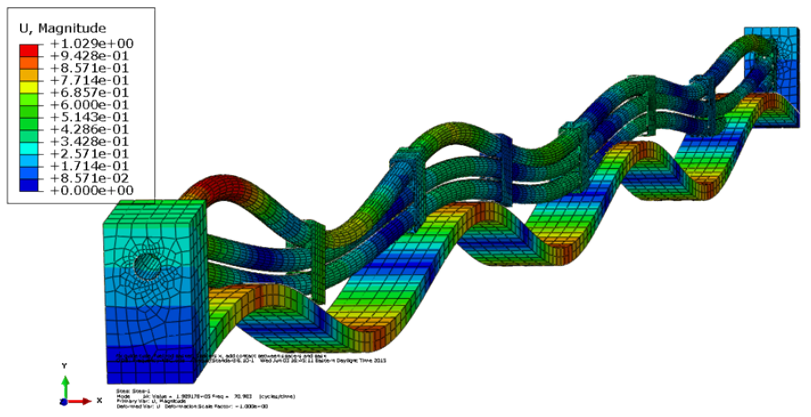

Mode-12: $70.983 \mathrm{~Hz}$

Fig. 9b. The second 6 modes and the associated natural frequencies of the fuel assembly submodel. 
A modal analysis of the SNF assembly submodel was conducted using ABAQUS linear perturbation Frequency code, in which the system is modeled with a simply supported structure. The first 12 mode shapes and associated natural frequencies are shown in Fig. 28. The fundamental mode of this submodel has a natural frequency of $2 \mathrm{~Hz}$, which is considerably lower than that of the cradle/cask system models, with fuel assembly weights, described in Ross 2015 [5]. In the first 12 modes shown in Fig. 28, the guide tube and fuel rods are mostly synchronized (i.e., within the same phase), while the basket floor vibrates within a difference phase. When higher vibration modes occur, as shown in Fig. 29, system components such as the guide tube and fuel rods are no longer in the same phase. Local vibration motions are involved with different components, and in some modes (e.g., modes 16-18), the fuel rod vibrations become dominant.

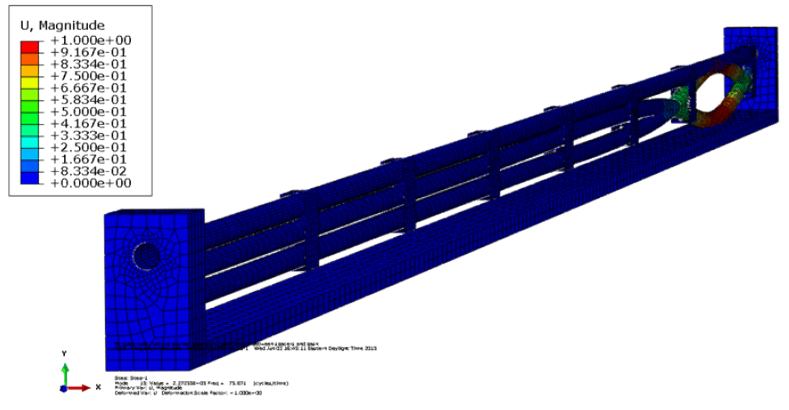

Mode-13: $75.871 \mathrm{~Hz}$

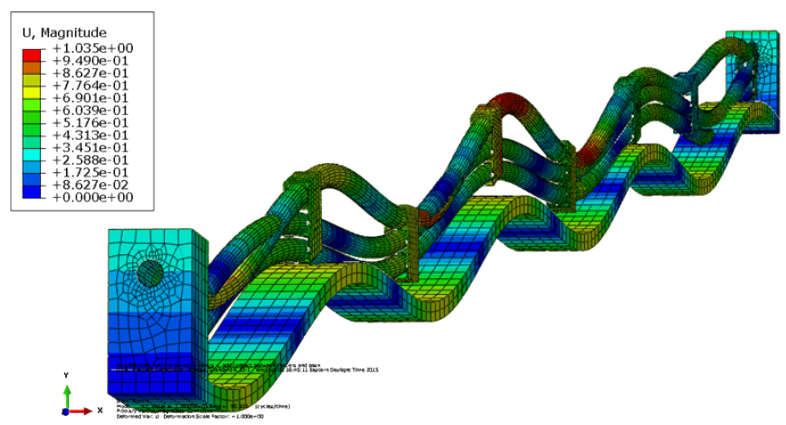

Mode-15: $90.103 \mathrm{~Hz}$

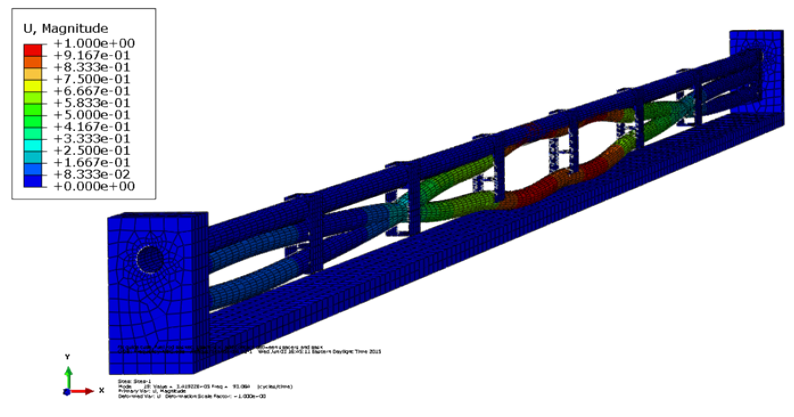

Mode-17: $93.064 \mathrm{~Hz}$

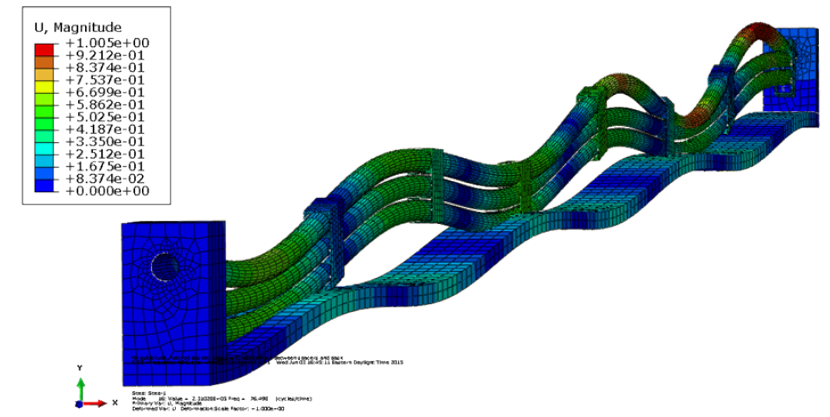

Mode-14: $76.498 \mathrm{~Hz}$

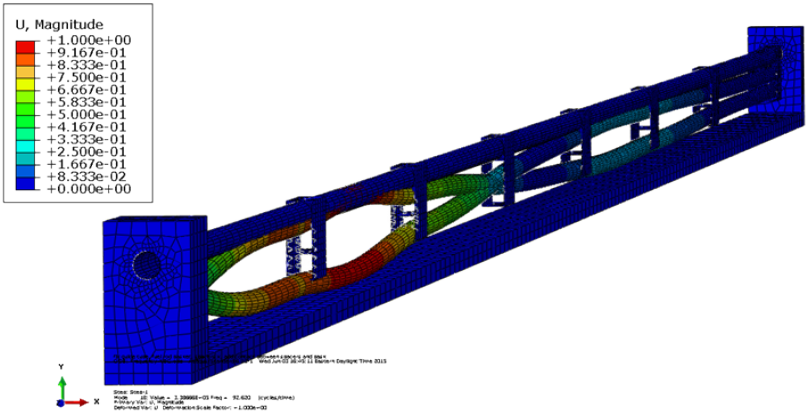

Mode-16: $92.62 \mathrm{~Hz}$

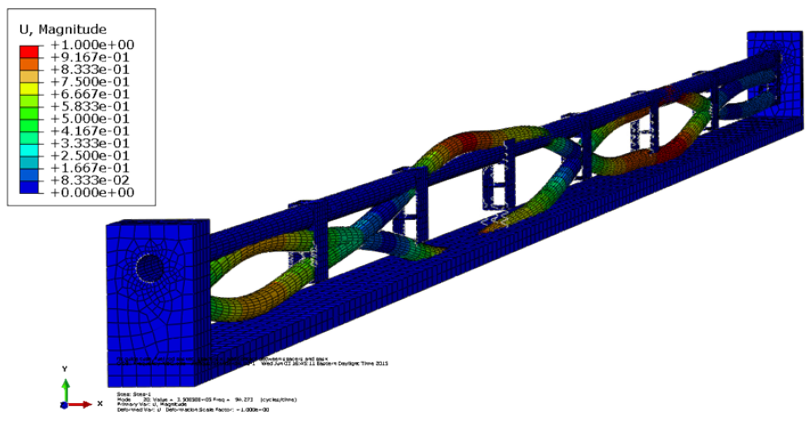

Mode-18: $94.273 \mathrm{~Hz}$

Fig. 29. Higher modes and associated natural frequencies of the fuel assembly submodel. 
Modal analysis results of the SNF fuel assembly submodel are summarized in Table 3 . The natural frequencies for the mode are listed up to mode 51, and the associated participation factor and effective mass are evaluated accordingly. The relative strength of each mode is ranked by the participation factors, which are normalized and listed as ratio values. The participation factor with the strongest mode is assigned a value of 1.0, and the other participation factors are normalized to that value. The participation of effective mass in the deformation of the mode shape is another way to indicate the strength of modes. Large mass indicates a stronger mode. The participation factor and effective mass correspond to each other. Table 3 shows that the strongest mode is the first mode's frequency of 2.444 $\mathrm{Hz}$. As shown in Fig. , the modes containing odd numbers of half sine wave have higher participation factor ratios. For example, mode 5's frequency of $14.792 \mathrm{~Hz}$ and mode 6's frequency of $20.845 \mathrm{~Hz}$ have 3 half sine wave shapes in the basket floor, the guide tube, and the fuel rods, respectively, where the participation factor ratio is in the second and third place. As discussed in Ross 2015 [5], a modal analysis was performed for a realistic cradle/cask system model and a concrete block model of equivalent mass. The results showed that the strongest mode for the cradle/cask system is at frequency of $52.4 \mathrm{~Hz}$. In the case of the concrete block model, the strongest mode is $318.1 \mathrm{~Hz}$, which is as high as the frequency of mode 49 for the fuel assembly submodel. The modal analysis indicates that system models with different structure level set-ups and different associated boundary conditions reveal significantly different natural frequency characteristics.

Table 3. Modal analysis of SNF assembly submodel

\begin{tabular}{cccc}
\hline Mode number & Frequency & $\begin{array}{c}\text { Participation } \\
\text { factor ratio }\end{array}$ & $\begin{array}{c}\text { Effective mass } \\
\text { (lbf s } \mathbf{s}^{\text {/in) }}\end{array}$ \\
\hline 1 & 2.444 & 1.000 & $8.195 \mathrm{E}-02$ \\
2 & 2.734 & 0.162 & $2.870 \mathrm{E}-03$ \\
3 & 5.826 & 0.004 & $1.246 \mathrm{E}-06$ \\
4 & 9.552 & 0.004 & $6.399 \mathrm{E}-07$ \\
5 & 14.792 & 0.381 & $1.079 \mathrm{E}-02$ \\
6 & 20.845 & 0.236 & $2.525 \mathrm{E}-03$ \\
7 & 22.665 & 0.011 & $1.037 \mathrm{E}-05$ \\
8 & 36.11 & 0.076 & $2.857 \mathrm{E}-04$ \\
9 & 37.702 & 0.221 & $3.965 \mathrm{E}-03$ \\
10 & 50.287 & 0.026 & $5.776 \mathrm{E}-05$ \\
11 & 55.525 & 0.182 & $1.419 \mathrm{E}-03$ \\
12 & 70.983 & 0.163 & $1.865 \mathrm{E}-03$ \\
13 & 75.871 & 0.001 & $6.431 \mathrm{E}-09$ \\
14 & 76.498 & 0.063 & $8.095 \mathrm{E}-05$ \\
15 & 90.103 & 0.026 & $4.378 \mathrm{E}-05$ \\
16 & 92.62 & 0.000 & $4.972 \mathrm{E}-10$ \\
17 & 93.064 & 0.000 & $4.257 \mathrm{E}-11$ \\
18 & 94.273 & 0.000 & $4.901 \mathrm{E}-10$ \\
$\ldots$ & & & \\
49 & 318.29 & 0.001 & $6.104 \mathrm{E}-09$ \\
51 & 345.44 & 0.014 & $6.133 \mathrm{E}-07$ \\
\hline
\end{tabular}




\section{A2.2 Transient Dynamic Analysis}

The objective of the transient dynamic analysis of the fuel assembly submodel was to determine the stress and strain states of submodel components in response to vibration loading of transient shock under NCT. Components included the fuel rods, guide tube, spacer grids, nozzles, and basket wall. The resulting stress/strain can be used in further component failure analyses. The contact interactions between fuel assembly system components during transient dynamic simulation were studied. The fuel assembly submodel contains numerous contact conditions and represents itself as a highly nonlinear system. The performed transient dynamic analysis used ABAQUS-explicit code in the time domain for nonlinear system evaluation.

Fig. 21d shows that there are 25 guide tubes for a $17 \times 17$ fuel assembly. Thus, there are 264 fuel rods contained in the spacer grid slots. It is assumed that the guide tube modeled at the edge of the full SNF assembly will roughly carry the gravity load of the neighboring 10 fuel rods. A gravitational acceleration loading of $386 \mathrm{in} / \mathrm{s} 2$ is applied to all components. The gravitation loads from the remaining 8 fuel rods are applied as the concentrated loads evenly distributed to eight spacer grids, as shown in Fig. 30. The developed dynamic simulation protocol consists of two stages. During the first stage, the basket wall is constrained, while the assembly (mainly the intermediate spacer grids) sags down due to the gravity load and comes to rest on the basket floor. During the second stage, the acceleration with a sine wave form or impulse signal to represent the normal vibration excitation or transient shock excitation that was applied to the basket and nozzles for transient dynamic analysis. In the second transient excitation stage, the constraint at the basket floor is removed, but the gravity loads are maintained for both stages of simulations. Therefore, the procedure for the subassembly model includes an initialization stage to establish contacts due to gravity followed by a transient dynamic loading stage. This procedure is similar to that provided in Ref. [2].

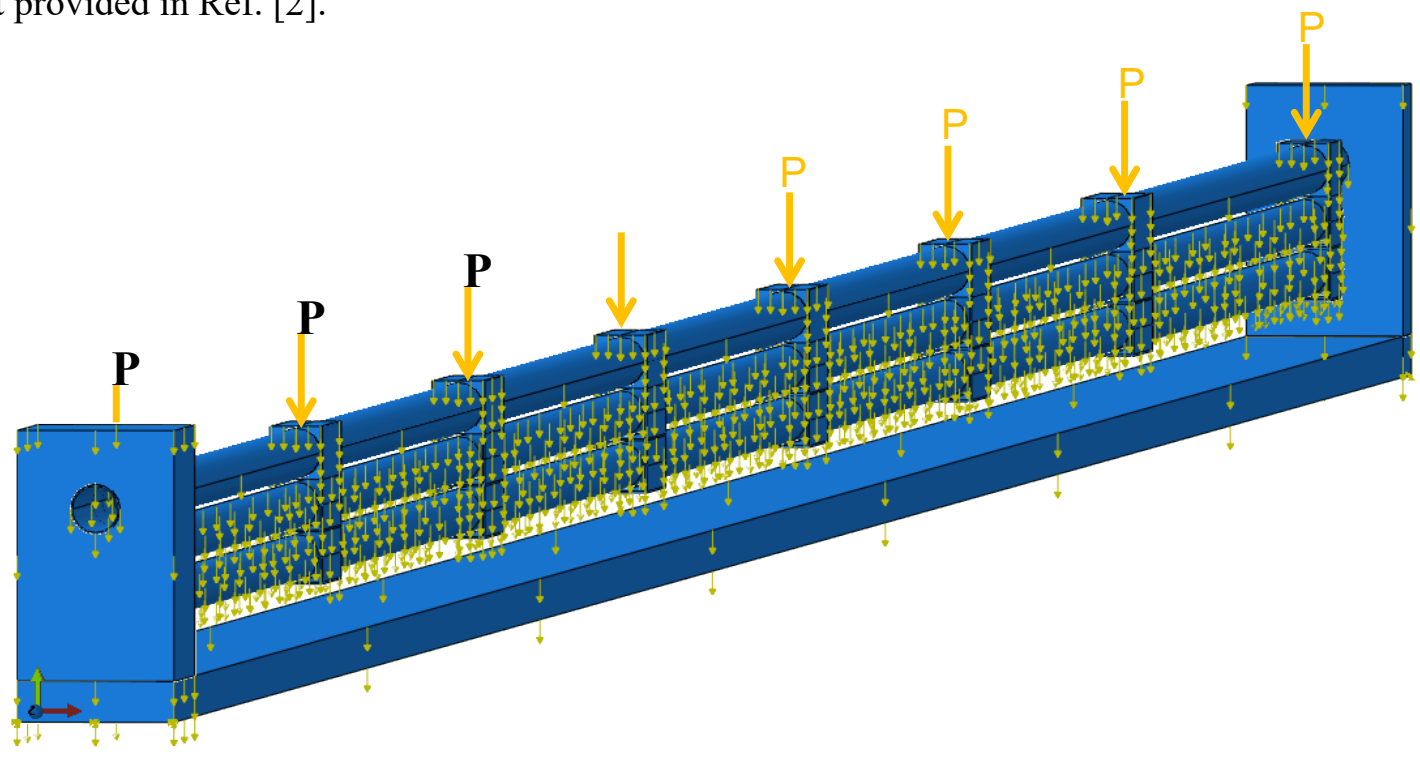

Fig. 30. Gravity loads for the initial stage of transient dynamic simulation of the fuel assembly submodel. 

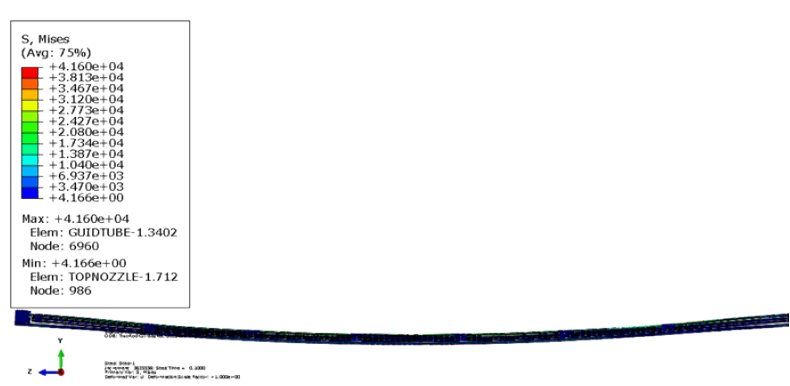

Guide tube and fuel rods sag in the middle

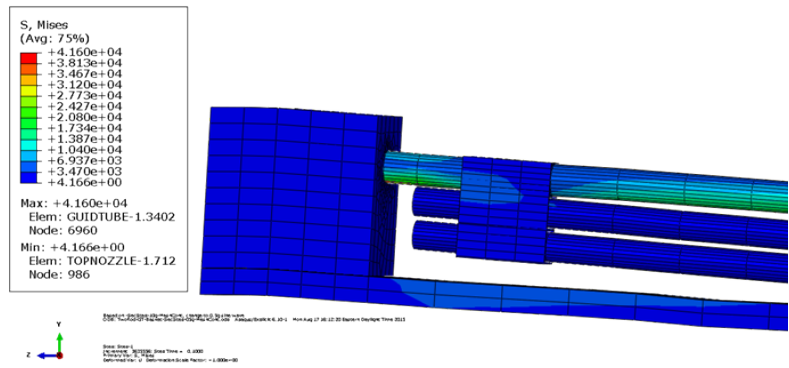

Top spacer grid doesn't contact basket floor
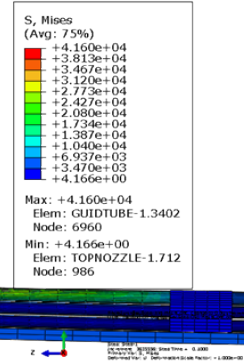

Intermediate spacer grids contact basket floor

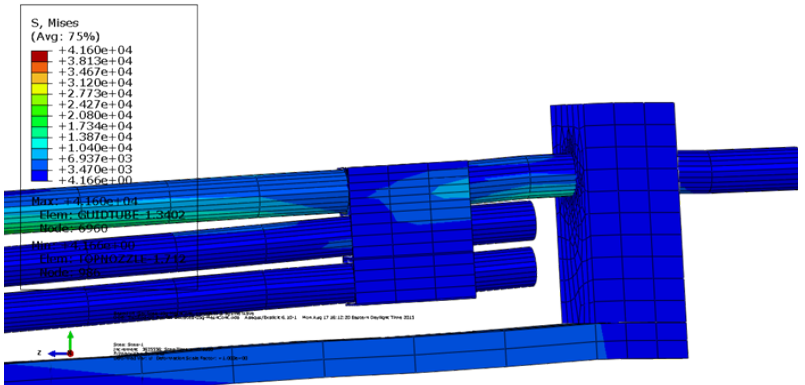

Bottom spacer grid doesn't contact basket floor

Fig. 31. Dynamic response of the fuel assembly submodel for the initialization stage.

Fig. 31 shows stress distribution and deformation of the SNF assembly submodel after the first step (initialization stage). During the initialization stage, only gravity load is applied to the system, and the basket floor is assumed to be simple supported at the cask. Maximum stress of $41.6 \mathrm{ksi}$ occurs at the guide tube. Maximum stress level is below all the material yield strengths listed in Table 2, so no yielding is observed in the system. The guide tube and fuel rods sag in the middle sections as a result of dead weight loads. Close-up views show that intermediate spacer grids rested on the basket floor after submodel assembly sagging stabilized. The top and bottom spacer grids did not contact the basket floor. Maximum contact pressure occurs at an intermediate spacer grid touching the basket wall, as shown in Fig. 32. Contact pressure level is relatively low because the fuel assembly gradually sags down to reach the basket floor, and the loading process was assigned as a ramp-up in conjunction with the quasistatic state loading condition during each loading interval. 


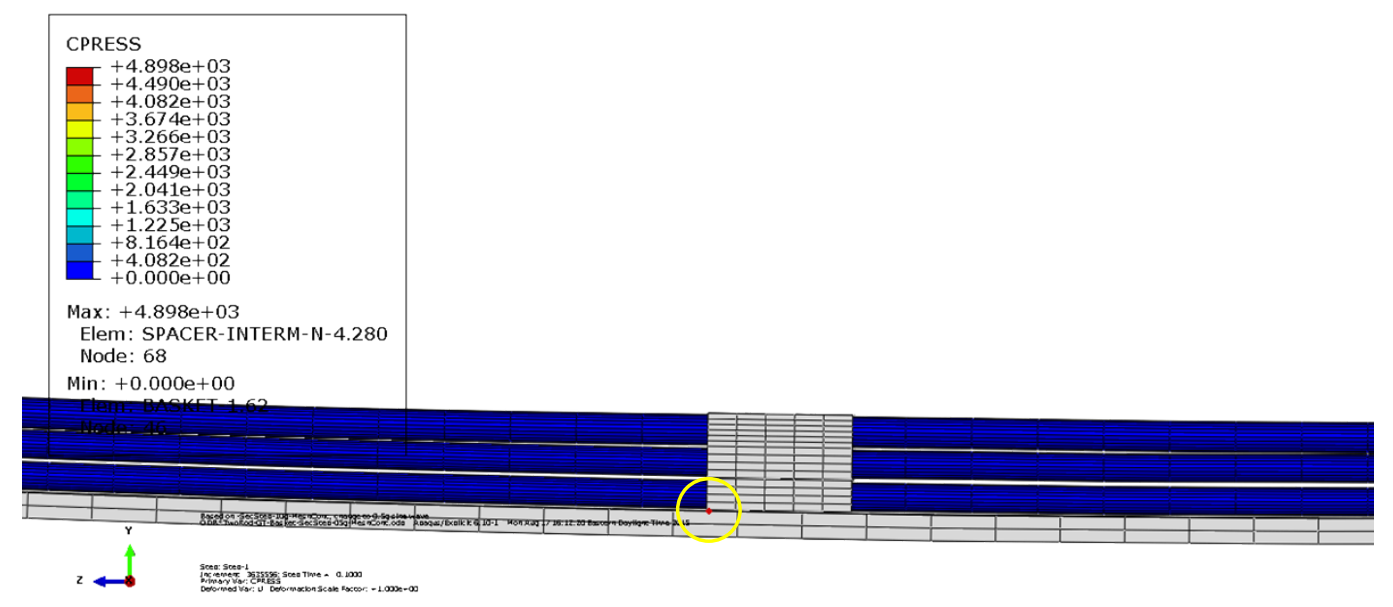

Fig. 32. Maximum contact stress resides at region where spacer grid in contact with basket floor for initialization stage.

The typical load-time history [6] registered by an accelerometer on a transport cask is shown in Fig. 33, which illustrates transient shock signals superimposed on continuous vibration signals of NCT. This random vibration from the cask provides the external loading to the SNF assembly. Inside the cask, another form of transient shocks comes from the dynamic impact induced by dynamic contact interactions between the fuel assembly components during NCT. These components include the canister basket wall, guide tube, fuel rods, and spacer grids.

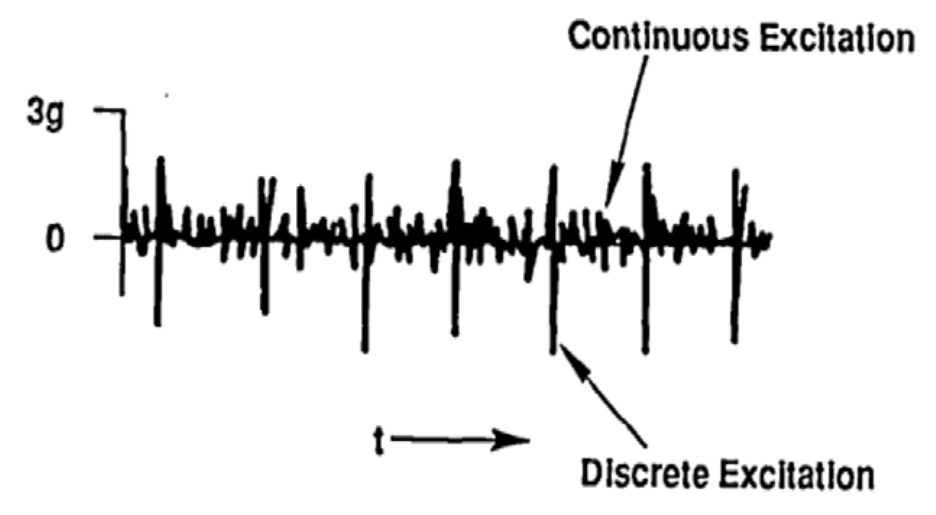

Fig. 33. Typical acceleration time history of a transport cask.

During the second stage, as shown in Fig. 34, gravity load remained, and the constraint on the basket wall was removed. The typical vibration load as acceleration-time history was applied to the basket and the top and bottom nozzles, exciting the SNF assembly submodel under normal vibrations or transient shocks of NCT. The first case of transient dynamics analysis is to simulate normal vibrations during NCT. Fig. 35 illustrates that a $0.5 \mathrm{~g}$ sine wave acceleration excitation was applied to the basket wall and the top and bottom nozzles of the fuel assembly submodel. The sine wave has a frequency of $5 \mathrm{~Hz}$. 


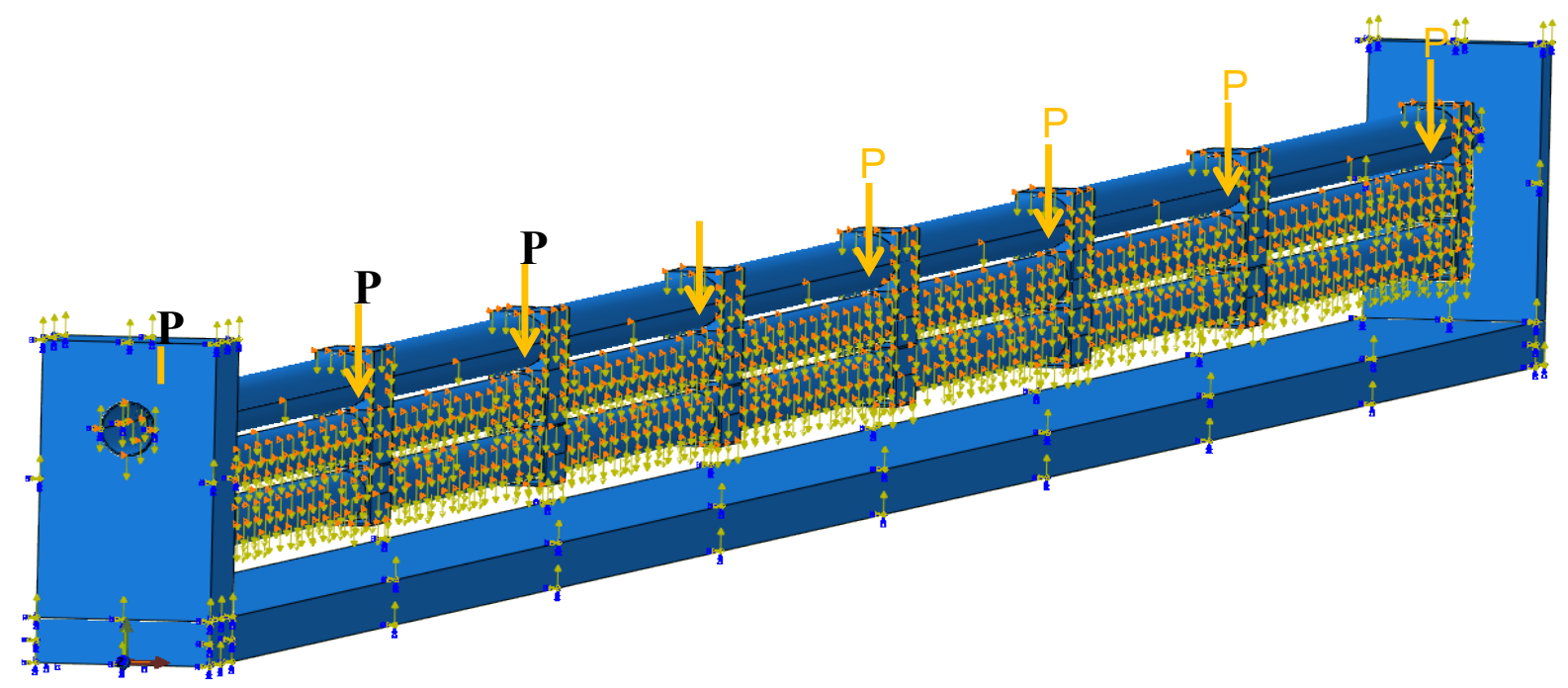

Fig. 34. Vibration boundary conditions and gravity system loads for the second stage of transient dynamic simulation of the fuel assembly submodel.

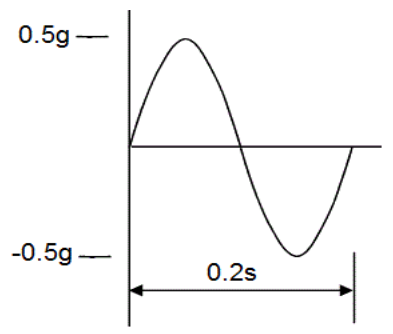

Fig. 35. A $0.5 \mathrm{~g}$ sine wave acceleration excitation used to simulate the normal vibration of NCT.

Fig. 36 shows the dynamic responses of the fuel assembly submodel under a vibration of $0.5 \mathrm{~g}$ acceleration amplitude. Stress level is higher than in the initial stage. After $0.5 \mathrm{~g}$ normal vibration excitation, the fuel rod, guide tube, spacer grids and basket wall deformed nonuniformly as compared to deformation during the initialization stage. Some intermediate spacer grids lost contacts with the basket wall. According to von Mises stress distribution, besides the fuel pellets, the maximum stress occurs at the bottom Inconel spacer grid section where the material yields. 


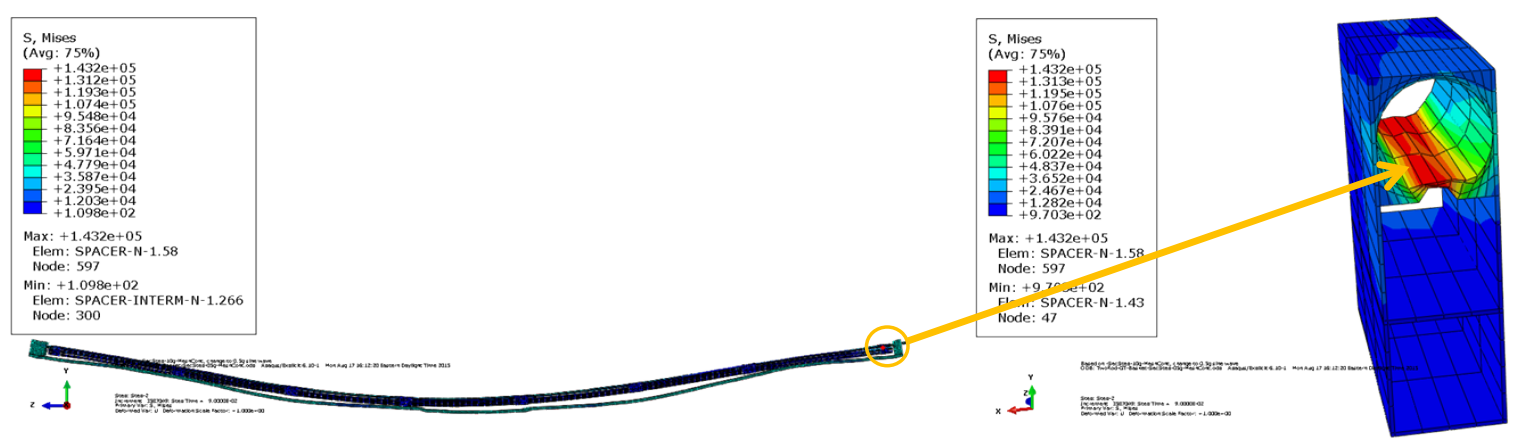

(a) Fuel assembly

(b) Bottom Inconel spacer

Fig. 36. The dynamic response of the fuel assembly submodel under $0.5 \mathrm{~g}$ sine wave acceleration excitation.

Due to the horizontal orientation of the fuel assembly during SNF transport, guide tubes and spacer grids become load bearing members to carry the dead weight and transmit the dynamic loads in the fuel assembly system. Therefore, spacer grids and guide tubes are responsible for load transferring within the fuel assembly during transport. The stress distributions in the guide tube are shown in Fig. 37. The maximum stress in the guide tube reaches the yield strength. Yielding on the guide tube occurs at both top and bottom nozzle locations. FEA results indicate that the guide tube and spacer grids are at high risk of failure. The failure of the skeleton will increase the random vibration contact frequencies among the fuel rods, and it will also increase their vibration intensity. Therefore, the integrity of the guide tube and spacer grids will critically affect the vibration modes and the vibration intensity of fuel assembly during transport.

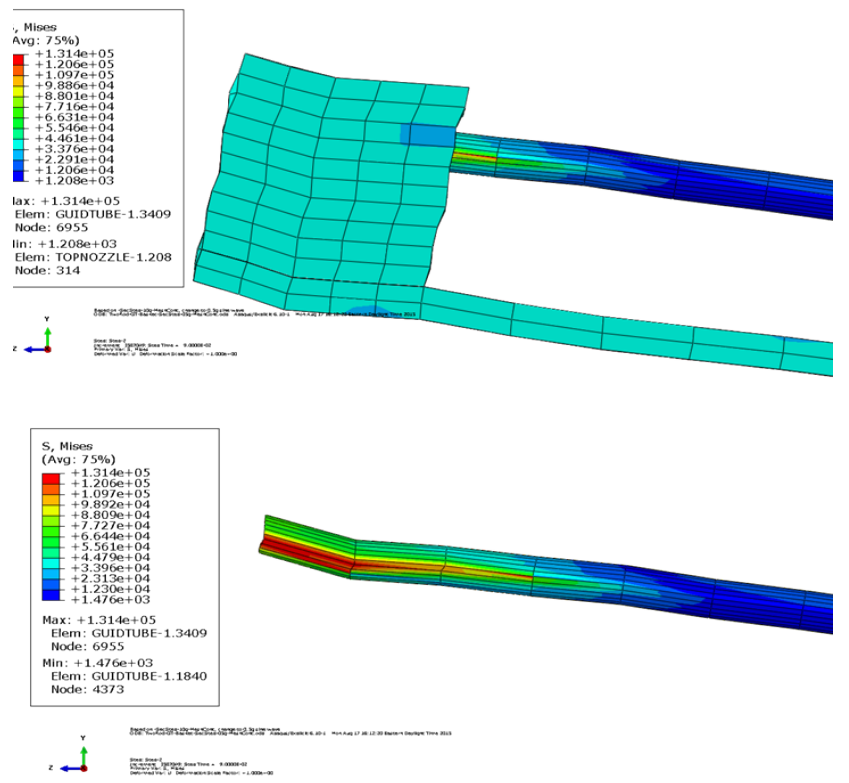

Top nozzle location

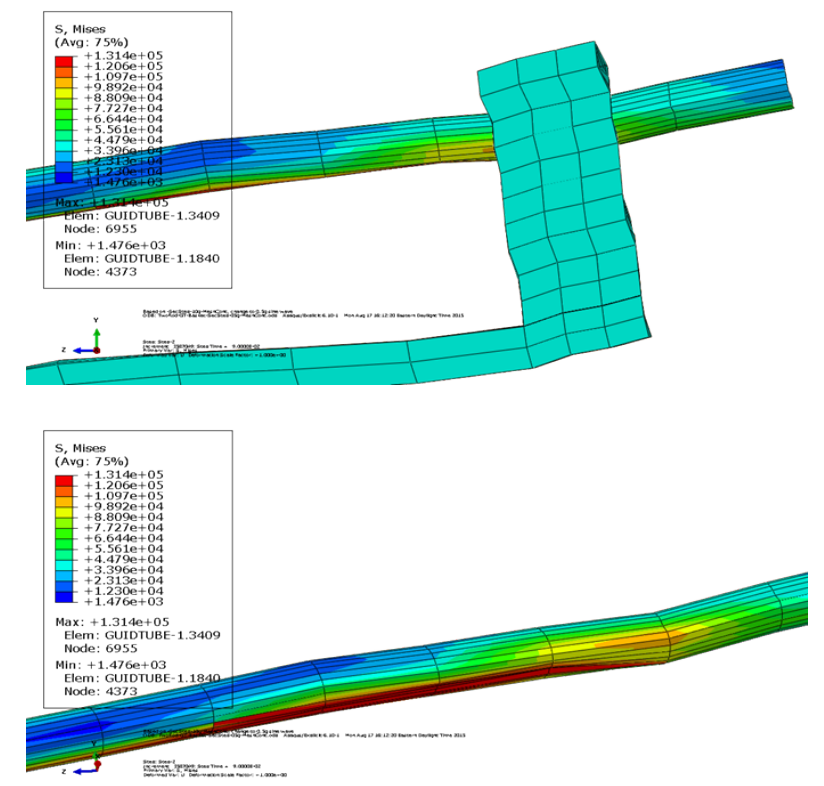

Bottom nozzle location

Fig. 37. The dynamic response of guide tube under $0.5 \mathrm{~g}$ sine wave acceleration excitation. 
Fig. 38 shows the stress distribution and deformation of other system components in the fuel assembly submodel (including the fuel cladding, the basket floor, and the top and bottom nozzles) with reference to the dynamic response of vibration under $0.5 \mathrm{~g}$ sine wave acceleration excitation. Under a $0.5 \mathrm{~g}$ simulated normal vibration load, the maximum stress of claddings is below the material yield strength, so the claddings remain intact. However, the basket wall and the top and bottom nozzles were yielded, possibly due to the transient shocks and/or impact load induced by the contacts' interaction between the spacer grids, guide tube, and fuel rods.

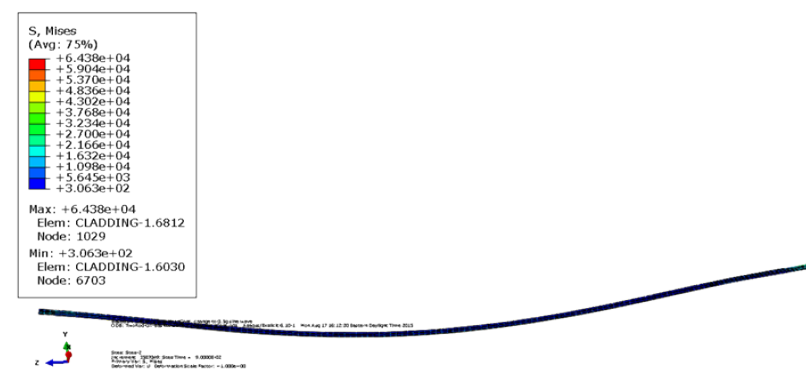

Claddings

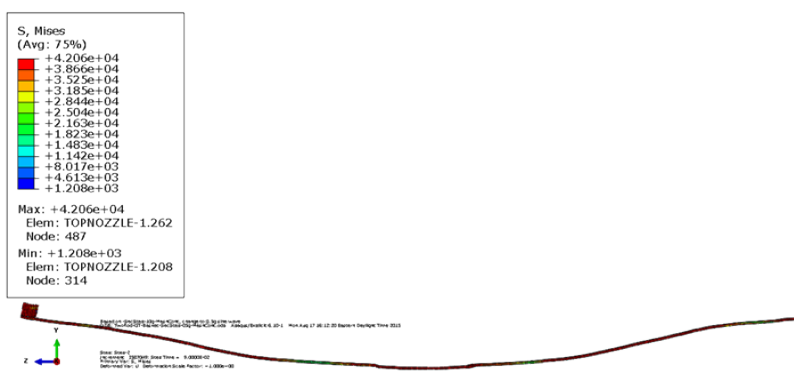

Basket wall, top and bottom nozzles

Fig. 38. The dynamic response of cladding, basket floor, top and bottom nozzles under $0.5 \mathrm{~g}$ sine wave acceleration excitation.

Fig. 39 shows that the maximum contact pressure of the SNF assembly submodel under $0.5 \mathrm{~g}$ sine wave acceleration excitation is located at the contact point of the spacer grid and the basket floor. When compared to the contact point in the initialization stage, the maximum contact pressure level is almost one order of magnitude higher. However, the maximum contact pressure is still under material yield strength.

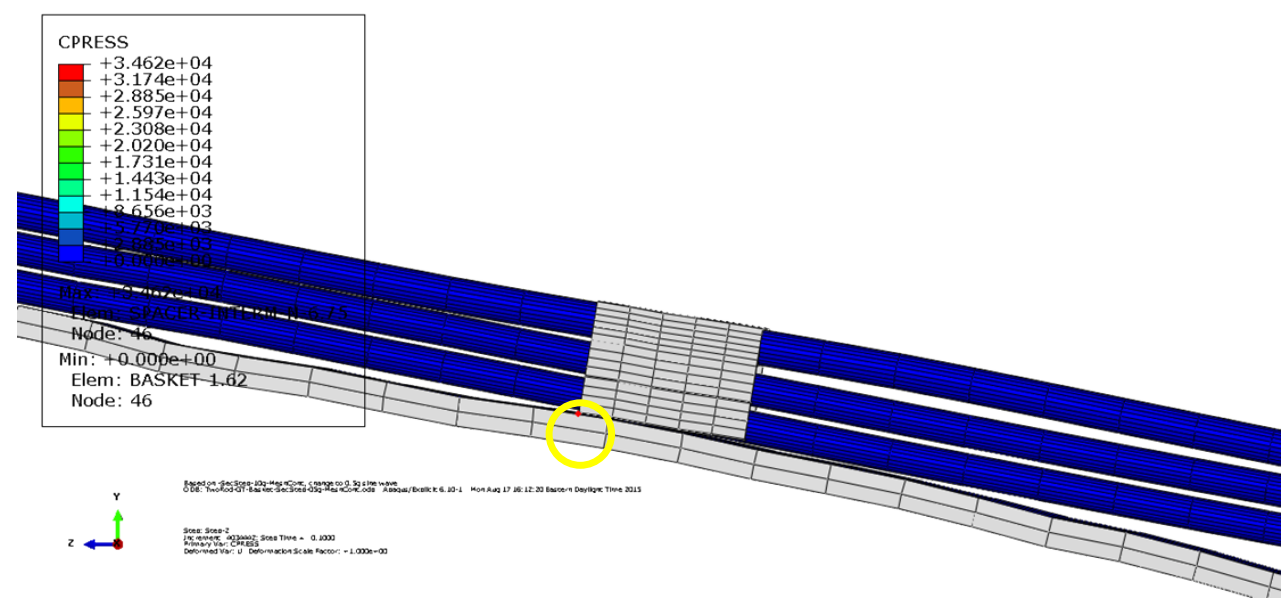

Fig. 39. Maximum contact pressure under $0.5 \mathrm{~g}$ sine wave acceleration excitation resides at the contact point of the spacer grid and the basket floor, as marked with a yellow circle. 
The second phase of transient dynamics analysis is to study the dynamic response of the SNF assembly submodel under transient shock loading during NCT. According to Fig. 33, a 3 g periodic impulse acceleration excitation was simulated as transient shock during transportation, as shown in Fig. $\mathbf{4 0}$. It is assigned a peak value of 3 times gravity and a duration period of $1 \mathrm{~s}$. The resulting system dynamic responses within this loading cycle period are shown in Fig. 41. Similar to the dynamic response of $0.5 \mathrm{~g}$ sine wave acceleration excitation, yielding occurs at bottom Inconel spacers during $3 \mathrm{~g}$ transient shock excitation, as shown in Fig. 41 . Fig. 42 reveals that the guide tube also yields at the top and bottom nozzle locations during $3 \mathrm{~g}$ transient shock loading. Therefore, the integrity of the spacer grids and guide tube are the concern under such transient shock loading during NCT.

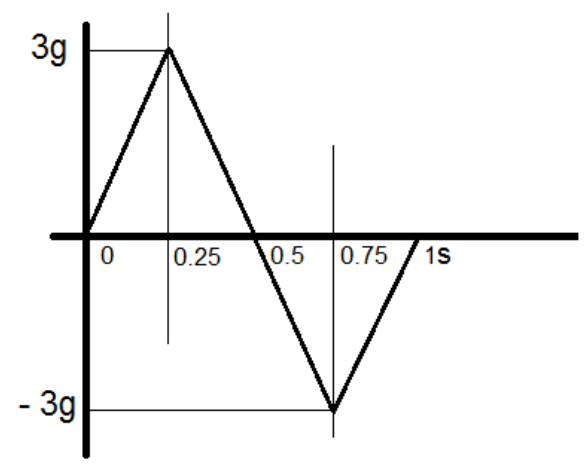

Fig. 40. A $3 \mathrm{~g}$ impulse acceleration excitation time-history representing the transient shock of NCT.

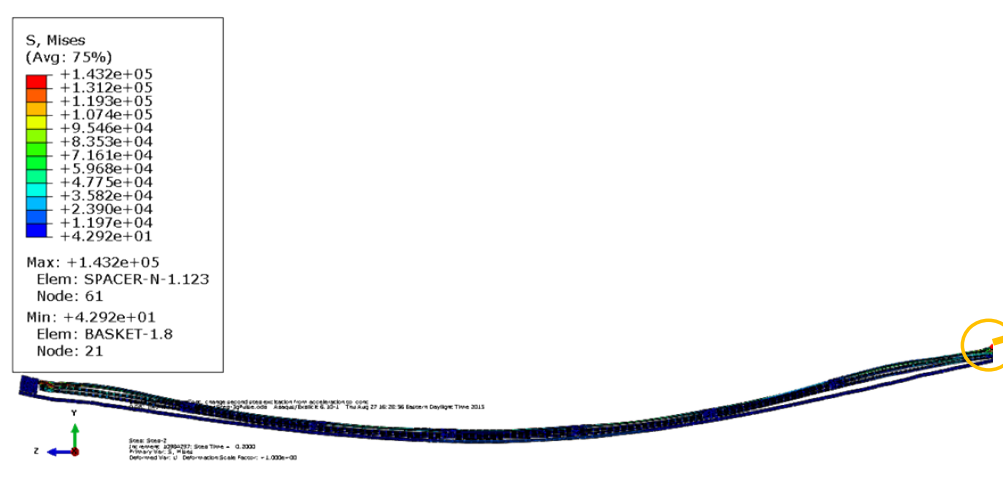

Fuel assembly

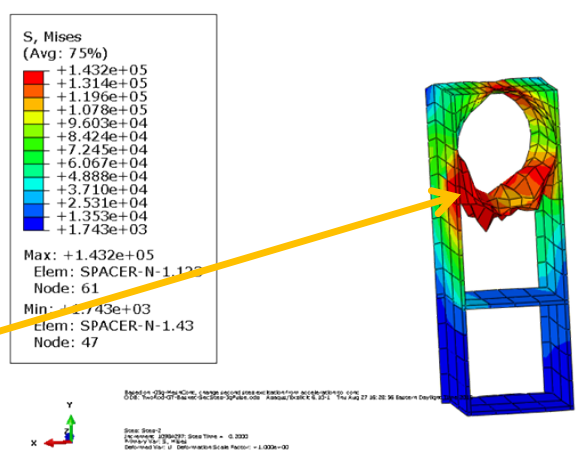

Bottom Inconel spacer

Fig. 41. The dynamic response of the fuel assembly submodel under $3 \mathrm{~g}$ transient shock excitation. 

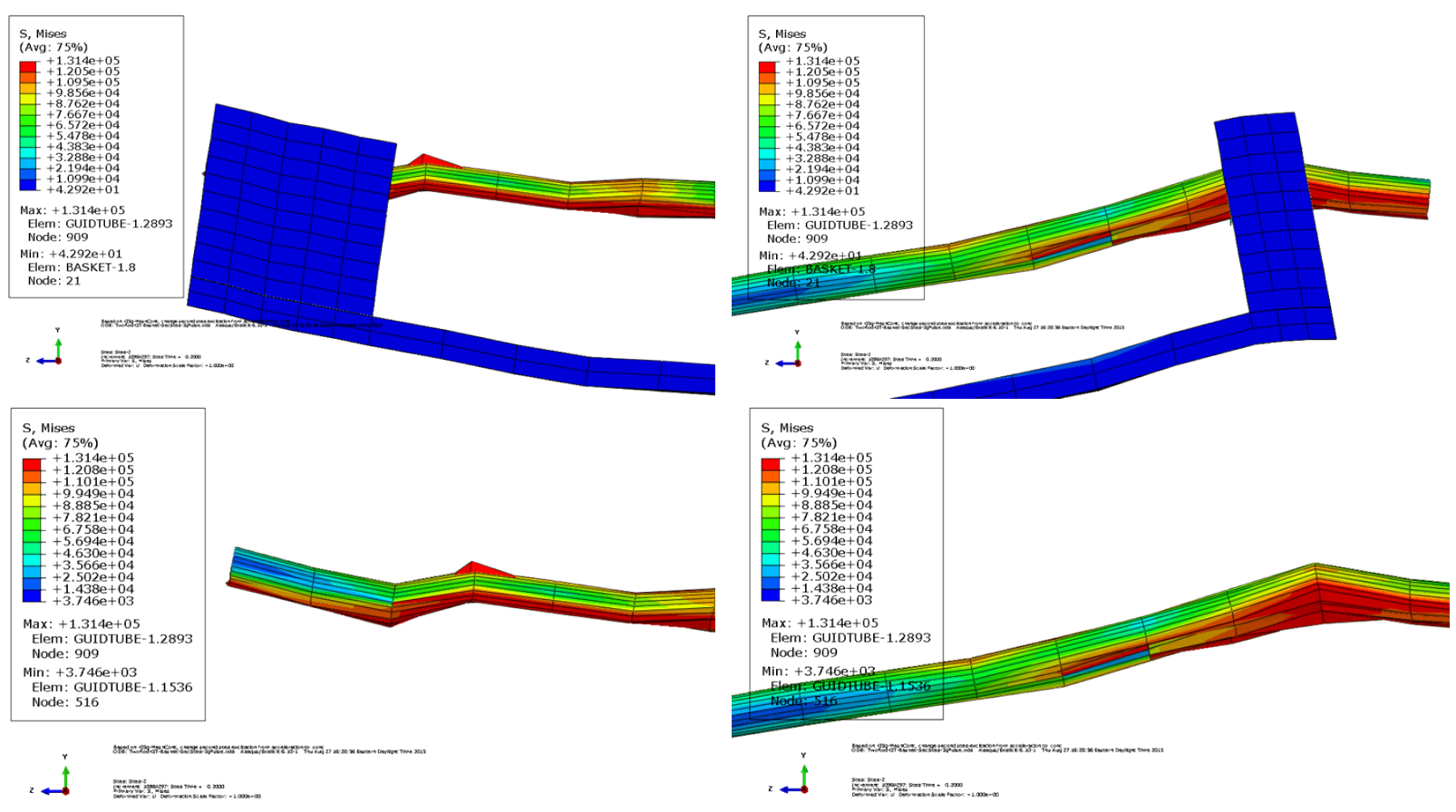

Top nozzle location

Bottom nozzle location

Fig. 42. The dynamic response of guide tube under $3 \mathrm{~g}$ transient shock excitation.

The dynamic response of claddings under $3 \mathrm{~g}$ transient shock excitation is shown in Fig. 43. The FEA result indicates that the maximum stress level is twice that of $0.5 \mathrm{~g}$ sine wave acceleration excitation and is near the yield strength. The basket wall and the top and bottom nozzles also have regions that reach their associated yield strengths. 


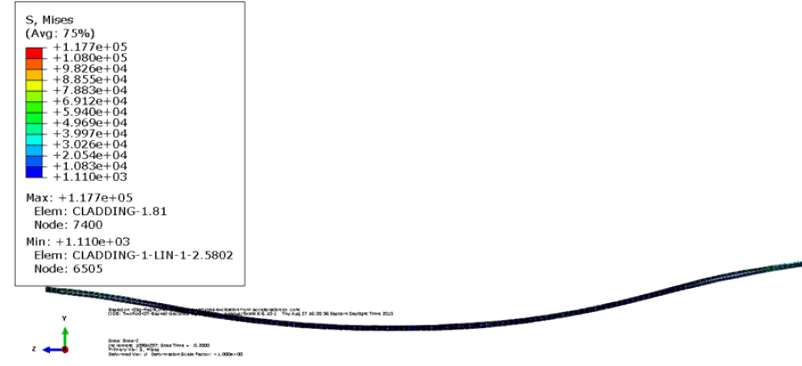

Claddings

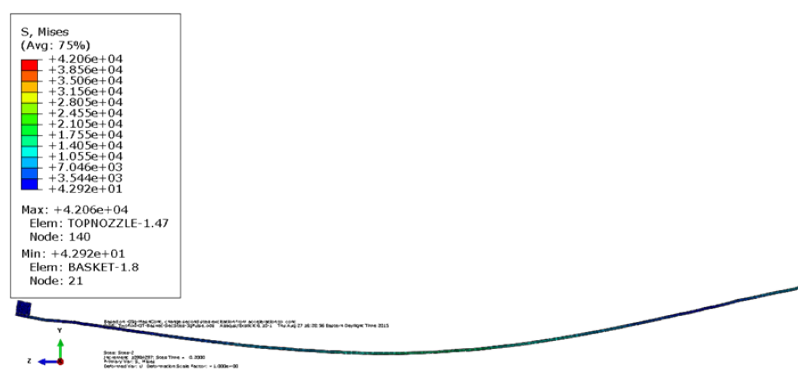

Basket, top and bottom nozzles

Fig. 43. The dynamic response of the cladding, basket wall, and top and bottom nozzles under $3 \mathrm{~g}$ transient shock excitation.

In the transient shock excitation case, the maximum contact pressure occurs at the location where the guide tube is in contact with the top fuel rod as shown in Fig. $\mathbf{4 4}$ (marked with a yellow circle). The FEA result indicates that the maximum contact pressure level is pretty high at this point. This type of dynamic contact interaction will inevitably form another pattern of transient shock loading within the fuel assembly system. Therefore, the contact interaction in the fuel assembly system, that can introduce transient shock loadings and impact SNF vibration integrity, needs more thorough investigation.

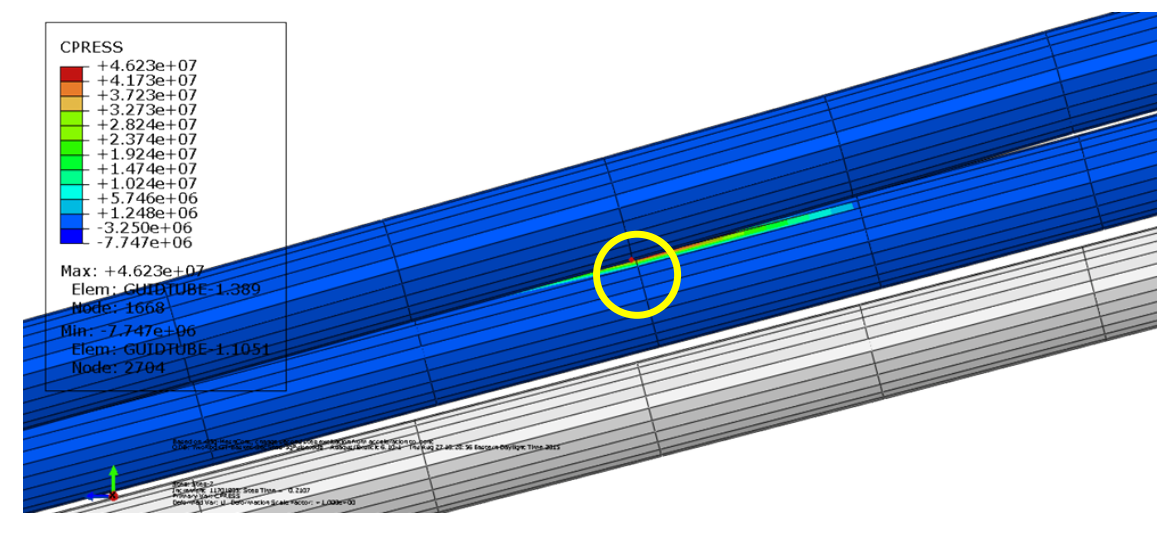

Fig. 44. Maximum contact pressure of $3 \mathrm{~g}$ transient shock excitation is located at the contact point of guide tube and top fuel rod, marked with yellow circle. 
The primary focus of this chapter is to estimate the interaction intensity at contact points between a fuel rod and spacer grids induced by dynamic impact loading. Due to the complex design of the fuel assembly system, a simplified scheme was necessary to enable a practical solution in the FEA effort; for instance in FEA modeling, the leaf springs and dimples of a spacer grid were modeled as translational springs in the earlier dynamic simulation effort. Fuel rods are connected to the spacer grid through these modeled translational springs. In Chapter 2's effort, the SNF assembly submodel simplified the spacer grid structure and ignored the contact interaction between the fuel rods and the spacer grids. However, a 3-D section model of the detailed leaf spring and dimples was built to evaluate contact interaction between the fuel rod and spacer grids induced by the impact loading from cask vibration during NCT.

Fig. 4 and Fig. 5 provide the details of a 3-D model of the fuel rod section with the dimples and leaf spring structures at the spacer grids. The fuel rod length is equivalent to a quarter of the length between the spacer grids. According to the targeted spacer grid design, there are two dimples on one side of a slot and one spring on the opposite side of the slot. Each dimple and each spring is an elongated member which arches from the associated grid strap inside the associated slot. Two dimples are modeled as arch-shaped members, where the flat section of the dimple is aligned with the fuel rod's surface contour in contact. Two dimples separate along the fuel rod axial direction at a distance of $0.021 \mathrm{~m}$. One leaf spring is modeled as an arch-shaped member, which is 45 degrees diagonal to the fuel rod axial orientation. The surface profile spacer grip region was obtained from a precision electronic scanning instrument. The sketch was implemented in ABAQUA CAE to build the spring/dimple structure geometry. The material of the spring/dimple is Zircaloy-4with a thickness of $0.47 \mathrm{~mm}$. The fuel rod material properties used in this chapter for all simulations, calculations, and results, as seen in Table 4.

The general contact algorithm was assigned at interfaces between the clad's bottom surface and the top surfaces of the dimples and spring. The ABAQUS dynamic explicit code was used in this impact response analysis. The approach was to simulate the impact load induced by the fuel rod inertia onto the dimples and spring within the spacer grids with a target impact velocity. Upon dynamic contact of the fuel rod and the dimples/spring, the contact stress and deformation of the interacting components (cladding, dimples, and spring) evolved progressively. Reaction forces at the contact surfaces were then estimated to evaluate the loading intensity of the target impact event.
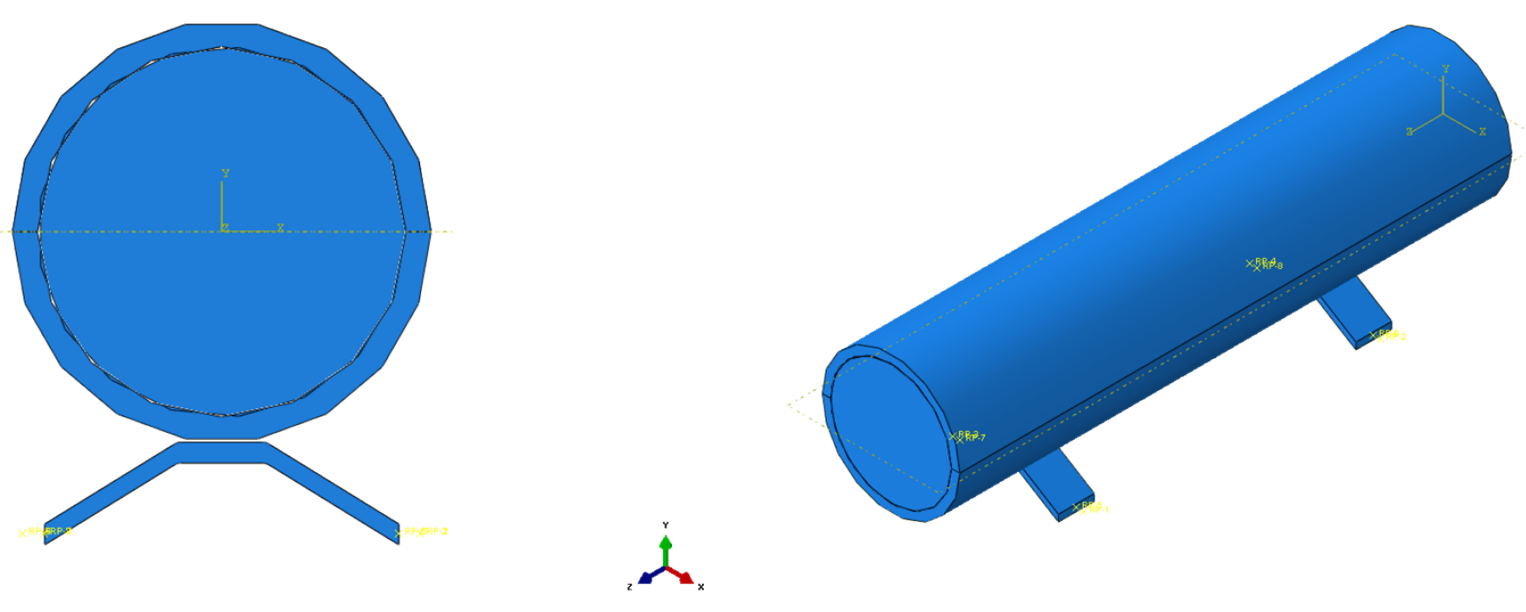
Fig. 45. Cross section view of the fuel rod with two dimples, modeled within the spacer grid region.
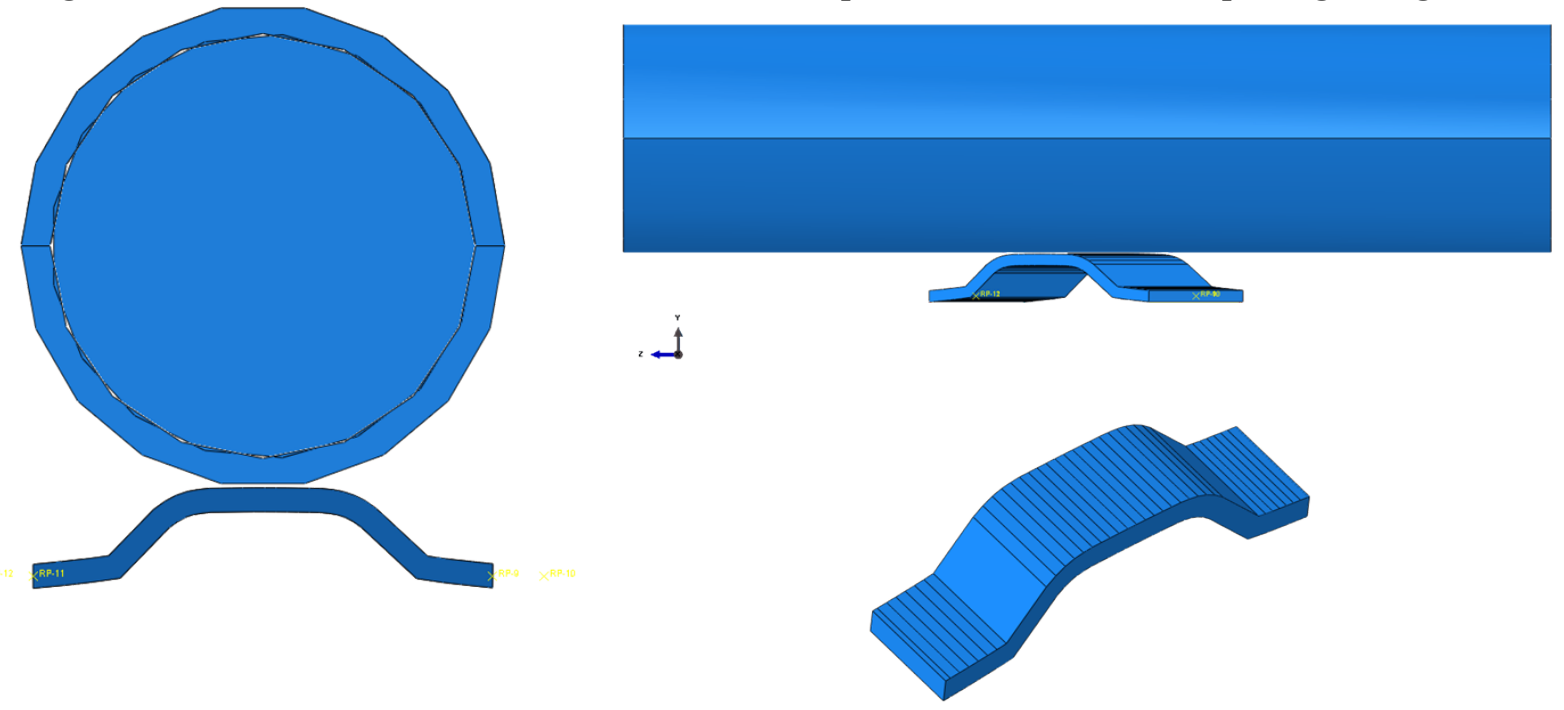

Fig. 46. Cross section view of the fuel rod with the leaf spring modeled at the spacer grid region.

Table 4. Mechanical properties of the fuel rod (SI)

\begin{tabular}{cccccc}
\hline & Material & $\begin{array}{c}\text { Young's modulus } \\
(\mathrm{GPa})\end{array}$ & $\begin{array}{c}\text { Poisson's } \\
\text { ratio }\end{array}$ & $\begin{array}{c}\text { Yield strength } \\
(\mathrm{MPa})\end{array}$ & $\begin{array}{c}\text { Density } \\
\left(\mathrm{kg} / \mathrm{m}^{3}\right)\end{array}$ \\
\hline Pellet & $\mathrm{UO}_{2}[3]$ & 201 & 0.32 & 2,146 & 10,970 \\
$\begin{array}{c}\text { Clad, } \\
\text { spring/dimple }\end{array}$ & Zircaloy-4 [3] & 91 & 0.33 & 906 & 6,560 \\
Block & Stainless steel [7] & 79.3 & 0.30 & 138 & 8,030 \\
\hline
\end{tabular}

During the truck transportation test performed at Sandia National Laboratory, the vibration load transferred from the cask to the fuel assembly has resulted in a maximum of approximately $20 \mathrm{~g}$ peak vertical vibration acceleration at the second spacer grid from the A7 accelerometer reading (Fig. 6). The FEA simulation protocol was developed and carried out to generate a quantitative estimate on the loading intensity experienced by ab SNF rod under such transient shock loading events. Practical assumptions were made based on the conservation of momentum and the system components' constraints to represent $20 \mathrm{~g}$ transient shock vibration periods for a fresh fuel assembly and an aging fuel assembly, including their associated initial contact velocities at the interface of the spacer grid and the fuel rod. In

Fig. 48, for a good fuel assembly skeleton system, the vibration time period was assigned to $0.04 \mathrm{~s}$, and the initial contact impact velocity of the fuel rod onto the spacer grid at $20 \mathrm{~g}$ acceleration is estimated at $0.98 \mathrm{~m} / \mathrm{s}$. For an aging skeleton system, the vibration time period was assigned to $0.2 \mathrm{~s}$, and the estimated initial contact impact velocity is at $4.9 \mathrm{~m} / \mathrm{s}$. The impact response time period is strongly dependent on the structural system stiffness and is the key factor to dictate the initial contact velocity during the transient shock of NCT. 


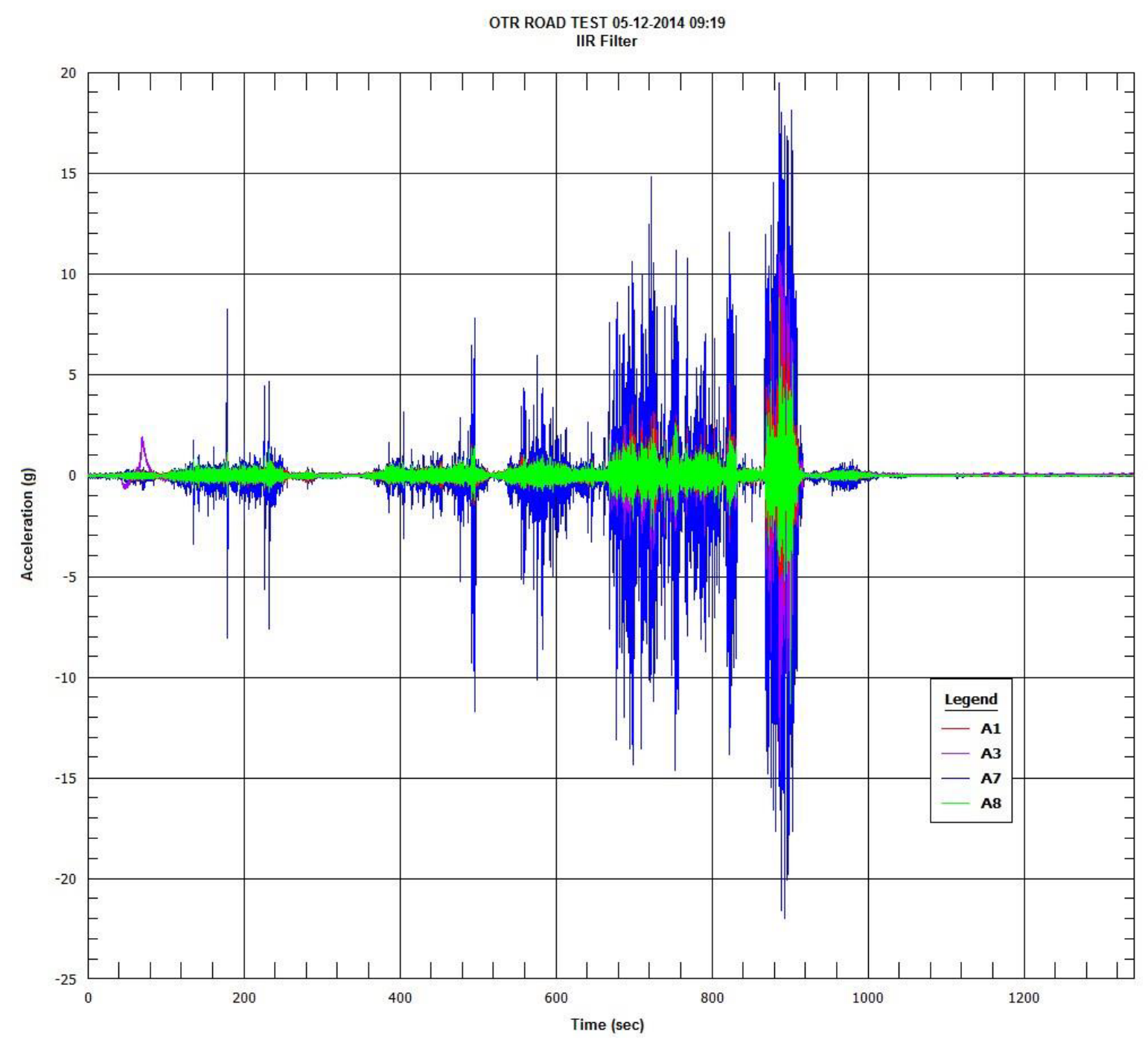

Fig. 47. Segment 1 accelerometer time-histories [8],

Momentum $=$ Mass $*$ Velocity $=\int F * d t, \mathrm{~F}=$ mass $*$ acceleration
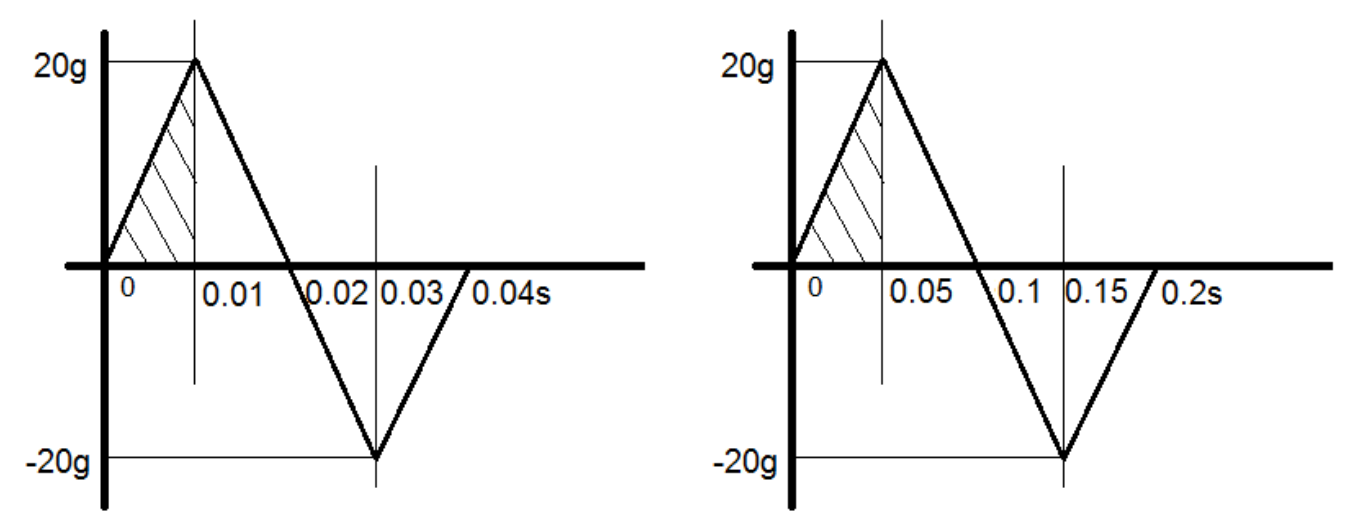
(a) Fresh fuel assembly system with good constraints among the fuel assembly skeleton system (b) Aging fuel assembly with less constraint functionality due to gaps' density increased among the fuel

Fig. 48. Schematics of $20 \mathrm{~g}$ acceleration transient shock periods for fresh and aging fuel assemblies showing an increased vibration cycle period assigned for loss of system constraints.

For instance, an aging fuel assembly structure would typically have lower system stiffness when compared to its virgin state due to the gaps increased in assembly's constraint skeleton system. This leads to an increase in the impact loading reaction cycle time period. To further investigate the impact contact interaction, the fuel rod and spacer grid's interaction was simulated with the dimples and leaf spring at the targeted initial contact velocity of $0.98 \mathrm{~m} / \mathrm{s}$ for a sound fuel assembly and $4.9 \mathrm{~m} / \mathrm{s}$ for an aging fuel assembly. Resulting contact stress profiles were compared to evaluate the transient shock impact effects under different scenarios.

Fig. 49 illustrates the progressive fuel rod impact on dimples at the initial velocity of $0.98 \mathrm{~m} / \mathrm{s}$. The impact mass of the fuel rod is limited within the $2 \mathrm{in}$. spacer grid section. The fuel rod traveled at the relative velocity of $0.98 \mathrm{~m} / \mathrm{s}$ to the spacer grid dimple. A fuel rod approximately $1.04 \times 10^{-3} \mathrm{~s}$ came into contact with dimples. The velocity of the fuel rod changed during progressive contact interaction. Due to the rod's initial momentum, it moved forward continuously against the dimples until its momentum reduced to zero at $1.11 \times 10^{-3} \mathrm{~s}$. At this point, the maximum stress of the dimple reached $752.8 \mathrm{MPa}$. Due to the dimple material's elastic recovery, the fuel rod started to bounce back. At $1.19 \times 10^{-3} \mathrm{~s}$, the contact stress level was reduced to that of the initial contact at $1.04 \times 10^{-3} \mathrm{~s}$. At $1.26 \times 10^{-3} \mathrm{~s}$, the fuel rod lost contact with the dimples, and the system's stress level further decreased. Residual stresses at the end of the impact event were low, indicating that under one such transient shock load cycle, the fuel rod and dimples largely remained intact with a slight increase in the dimple's permanent deformation (i.e., a slight gap increase between the fuel rod and the spacer grid).
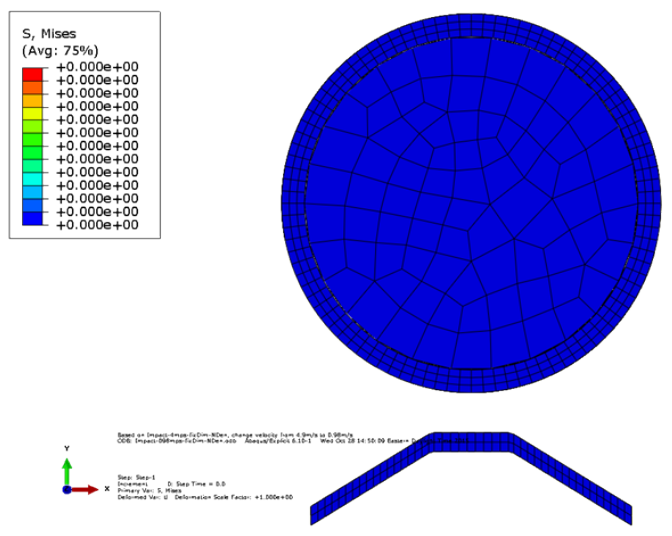

Original position

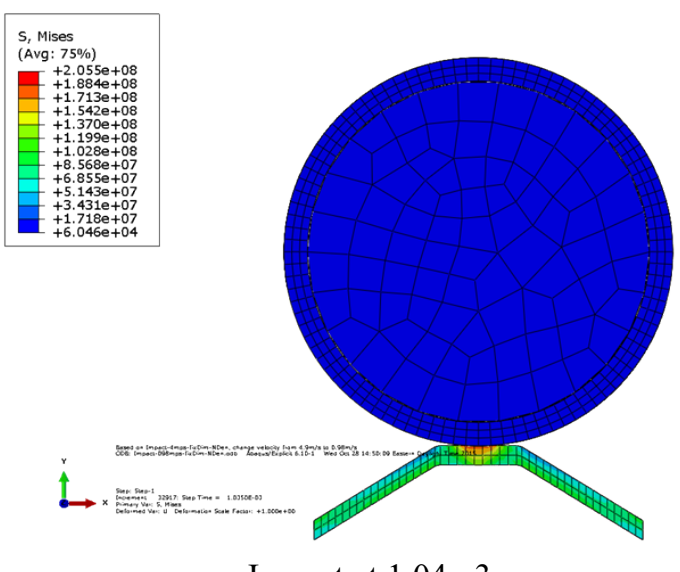

Impact at $1.04 \mathrm{e}-3 \mathrm{~s}$ 

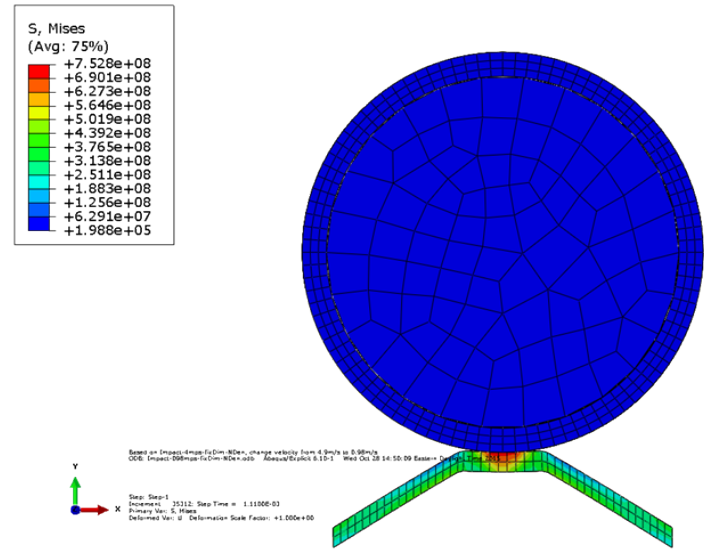

Impact at $1.11 \mathrm{e}-3 \mathrm{~s}$

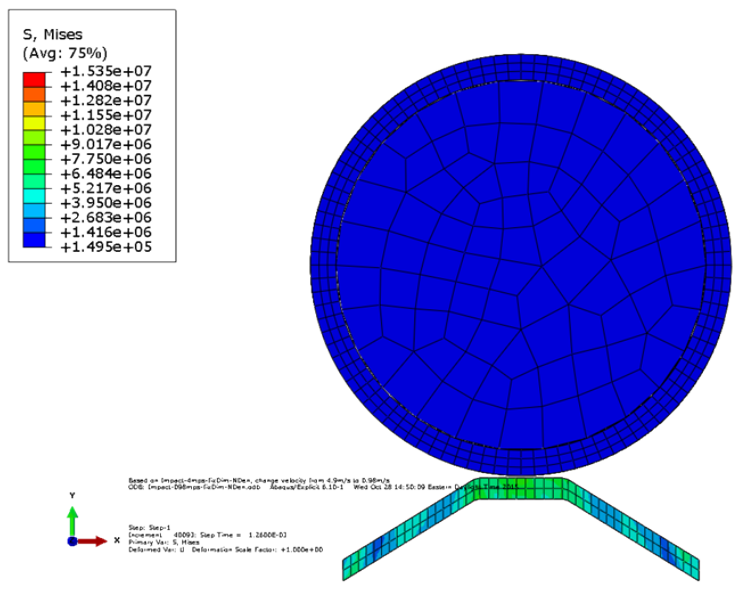

Impact at $1.26 \mathrm{e}-3 \mathrm{~s}$

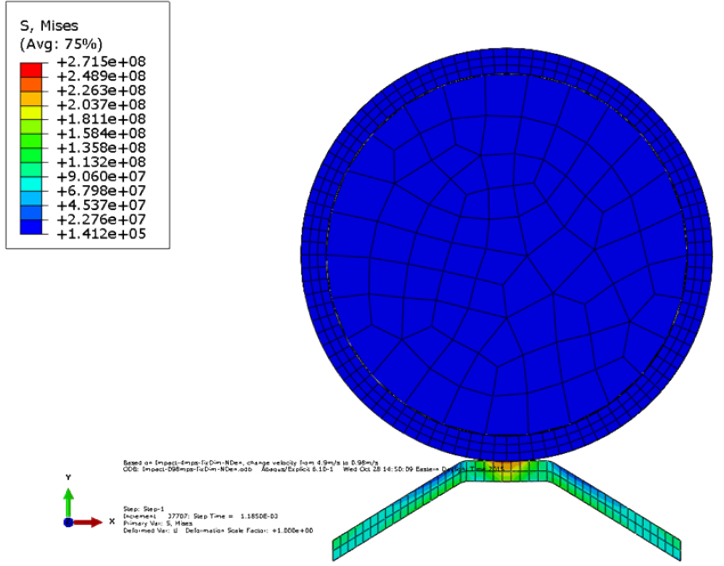

Impact at $1.19 \mathrm{e}-3 \mathrm{~s}$
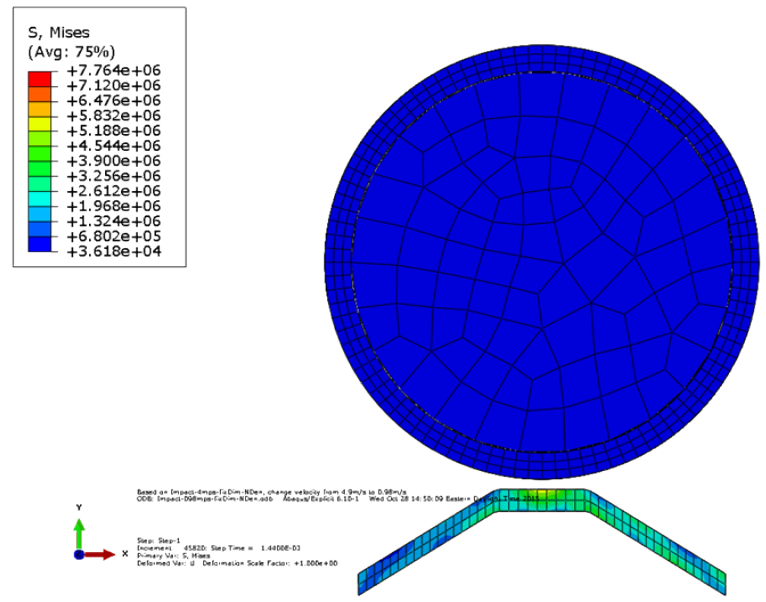

Impact at $1.44 \mathrm{e}-3 \mathrm{~s}$

Fig. 49. The progressive evolution of a fuel rod impact onto dimples at the velocity $\mathrm{of} 0.98 \mathrm{~m} / \mathrm{s}$.

Fig. 50 reveals that maximum stress occurs at dimples during the high speed impact. The maximum stress of $752.8 \mathrm{MPa}$ was reached in the dimples and is less than the yield stress. Thus, both the fuel rod and the dimples are not yielded. Under the cladding wall that is in contact with the dimples, the maximum stress of 254.5 MPa was reached on the inner surface of the cladding wall due to impact loading induced flexural bending tension stress on the inner wall of the cladding. 

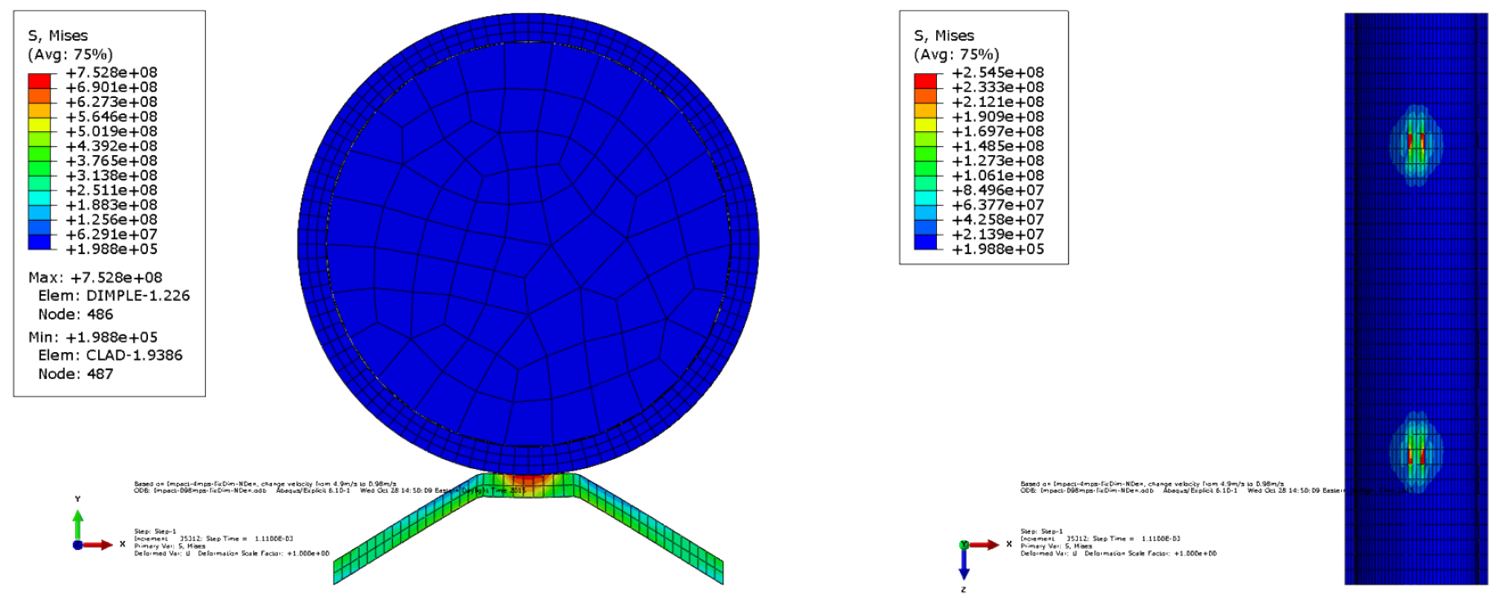

Fig. 50. Stress distribution of dimples and cladding during the fuel rod impact onto the dimples at the initial velocity of $0.98 \mathrm{~m} / \mathrm{s}$.

Fig. 51 shows that the highest contact pressure occurred on the contact surfaces of dimples and cladding during the impact. The highest impact contact pressure occurred at $1.11 \times 10^{-3} \mathrm{~s}$. Contact pressure on the dimples and cladding, both of which are under material yield stresses, are $270.8 \mathrm{MPa}$ and $323 \mathrm{MPa}$, respectively. The reaction force was calculated by integrating contact pressures over the associated contact areas on the dimples. The reaction force applied on one dimple is $291 \mathrm{~N}$.
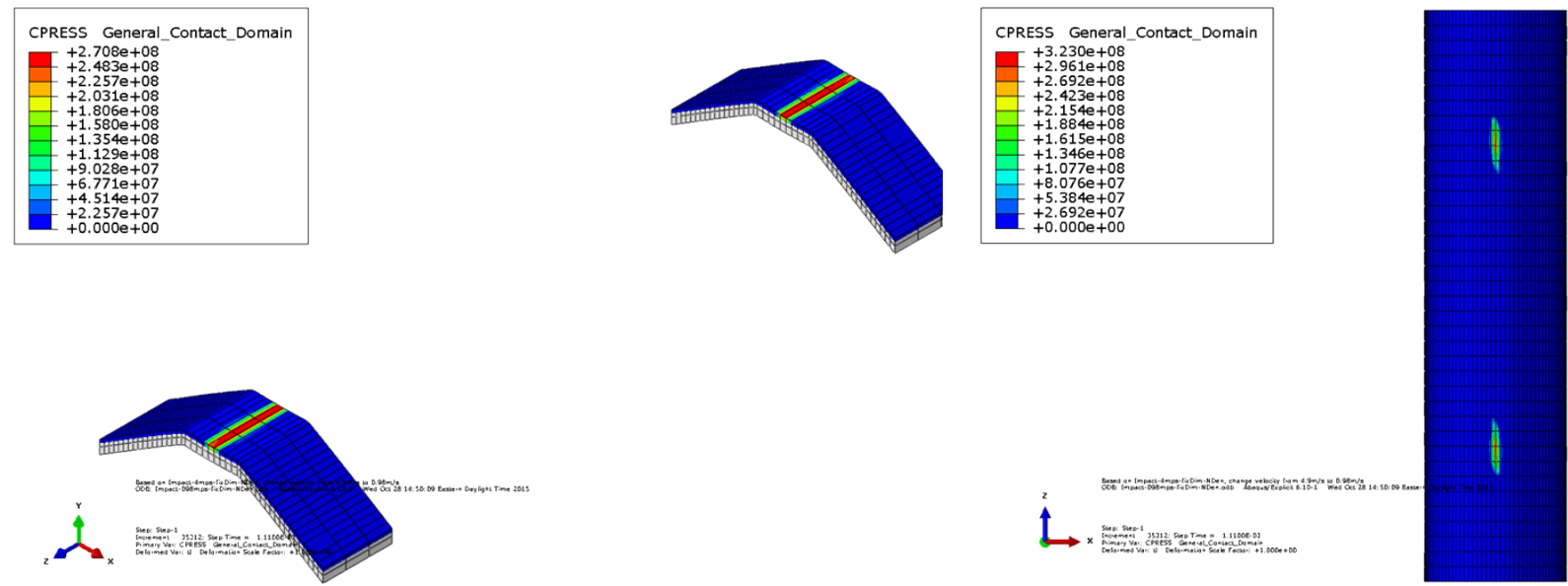

Fig. 51. Contact pressure on dimples and cladding during a fuel rod impact on dimples at the initial velocity of $0.98 \mathrm{~m} / \mathrm{s}$.

The fuel rod's impact on the dimple with the initial contact velocity of $4.9 \mathrm{~m} / \mathrm{s}$ was also investigated as shown in Fig. 52 and Fig. 53. The resulting contact stress profiles are much higher than that of impact velocity at $0.98 \mathrm{~m} / \mathrm{s}$, and the dimple was locally yielded under impact loading. The maximum stress on the cladding reached $386 \mathrm{MPa}$, so the cladding was not yielded. The maximum stress also occurred on the inner surface of the cladding wall due to impact-induced bending. The contact pressure levels on the dimples and cladding are higher than that of impact at the velocity of $0.98 \mathrm{~m} / \mathrm{s}$, but the contact pressure 
profiles of the system are still below material yield strength. The contact force estimated on one dimple is $371 \mathrm{~N}$, which is $27 \%$ higher than that of a lower speed impact case of $0.98 \mathrm{~m} / \mathrm{s}$.
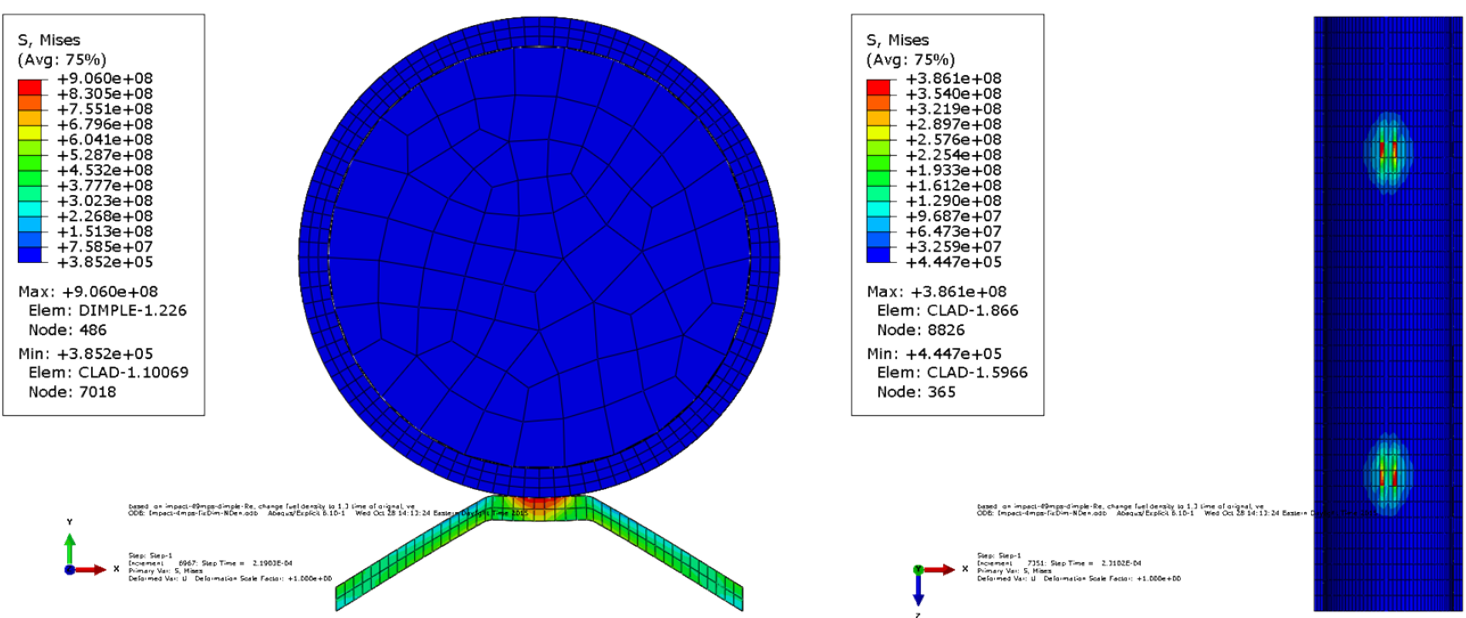

Fig. 52. Stress distribution profiles of dimples and cladding during the fuel rod impact on dimples at the initial velocity of $4.9 \mathrm{~m} / \mathrm{s}$.
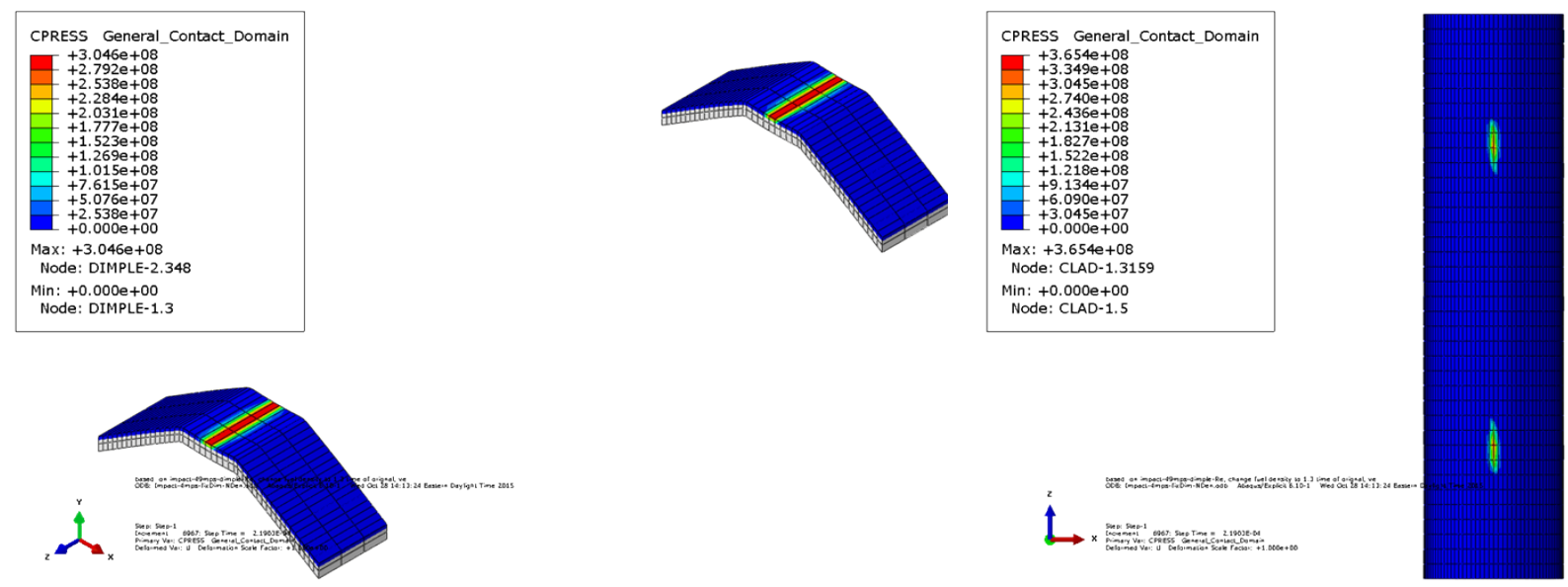

Fig. 53. Contact pressure on dimples and cladding during the fuel rod's impact on dimples at the initial velocity of $4.9 \mathrm{~m} / \mathrm{s}$.

Fig. 54 and Fig. 55 show the results for the fuel rod's impact onto the spring at the initial velocity of $0.98 \mathrm{~m} / \mathrm{s}$. The maximum stresses are $702 \mathrm{MPa}$ at the spring and $151 \mathrm{MPa}$ at the clad, where both the spring and the clad are not yielded. Compared to the stress of the fuel rod's impact onto the dimples at the same velocity, the stress level of the spring is relatively lower. This is because the spring is less stiff than the dimples. Contact pressure on the spring of $231 \mathrm{MPa}$ is higher than that on the clad of $140 \mathrm{MPa}$. In general, the spring's contact pressure is lower than that of the dimples with the same initial contact velocity. The estimated contact force on the spring is $357 \mathrm{~N}$, which is higher than the contact force on one dimple at $291 \mathrm{~N}$ for the same velocity impact. However, the contact force on the spring is less than the total contact force of two dimples at $582 \mathrm{~N}$. The contact pressure on the spring is also higher than the 
contact pressure on the dimples, but the reaction force on the spring is lower than the reaction force from both dimples.
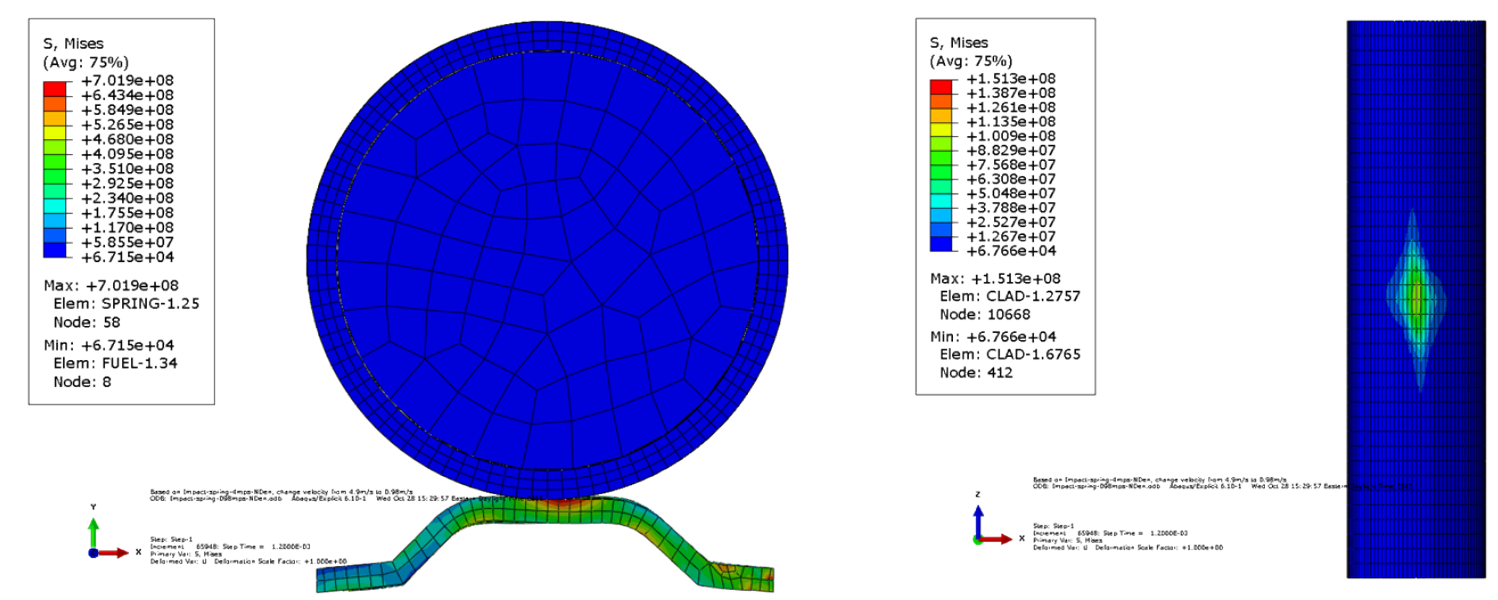

Fig. 54. Stress distribution of the spring and cladding during the fuel rod's impact on the spring at a velocity of $0.98 \mathrm{~m} / \mathrm{s}$.
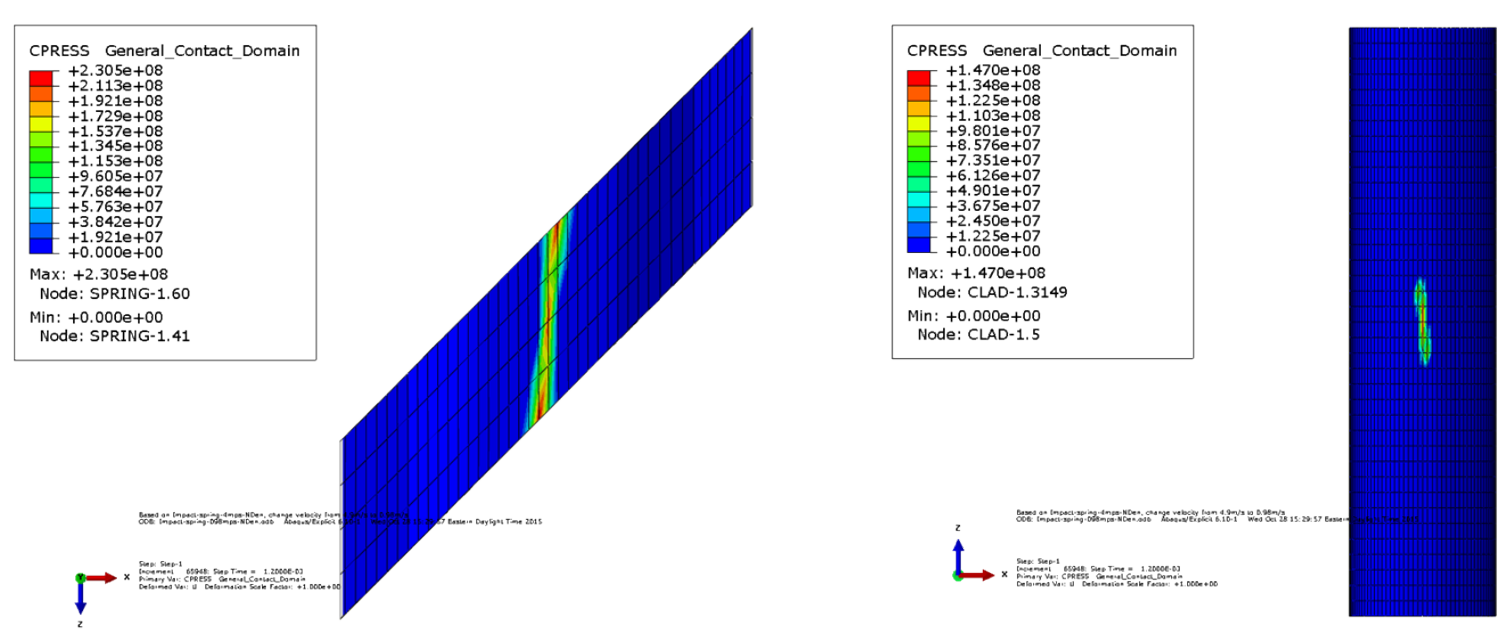

Fig. 55. Contact pressure on the spring and cladding during the fuel rod's impact on the spring at a velocity of $0.98 \mathrm{~m} / \mathrm{s}$.

Fig. 56 and Fig. 57 show FEA simulation results for the fuel rod's impact onto the spring at a velocity of $4.9 \mathrm{~m} / \mathrm{s}$. Similar to the fuel rod's impact on dimples at the same velocity, the spring is locally yielded during the impact event. The maximum stress of $190 \mathrm{MPa}$ on the clad, which is lower than the clad yield, is higher than that of the lower impact velocity case. This maximum clad stress of $190 \mathrm{MPa}$ is only half of that in the fuel rod and dimple impact case at the same impact velocity. The trends of the resultant spring contact pressure are similar among the two impact velocity cases. The maximum contact pressures on the spring and the clad are $298 \mathrm{MPa}$ and $191 \mathrm{MPa}$, respectively, both of which are below the material yield stresses. The estimated contact force on the spring for this impact case is $505 \mathrm{~N}$, which is $40 \%$ higher than that at lower speed impact of $0.98 \mathrm{~m} / \mathrm{s}$. 

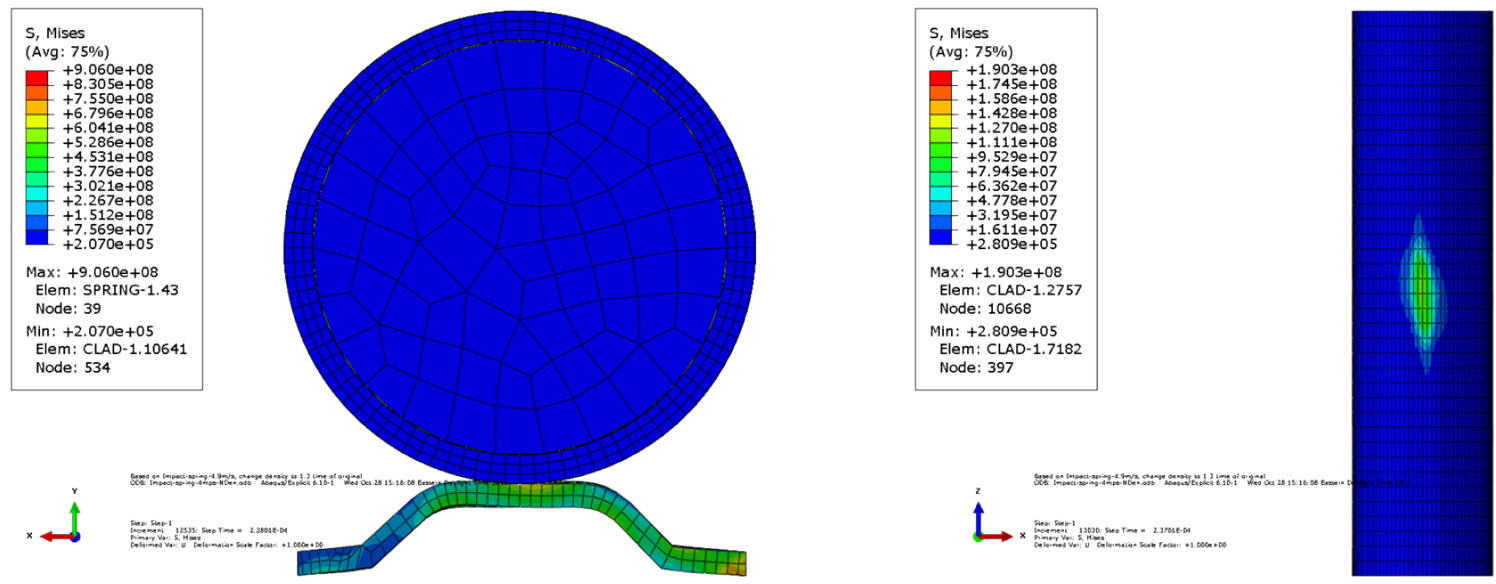

Fig. 56. Stress distribution of the spring and cladding during the fuel rod's impact on the spring at the initial velocity of $4.9 \mathrm{~m} / \mathrm{s}$.
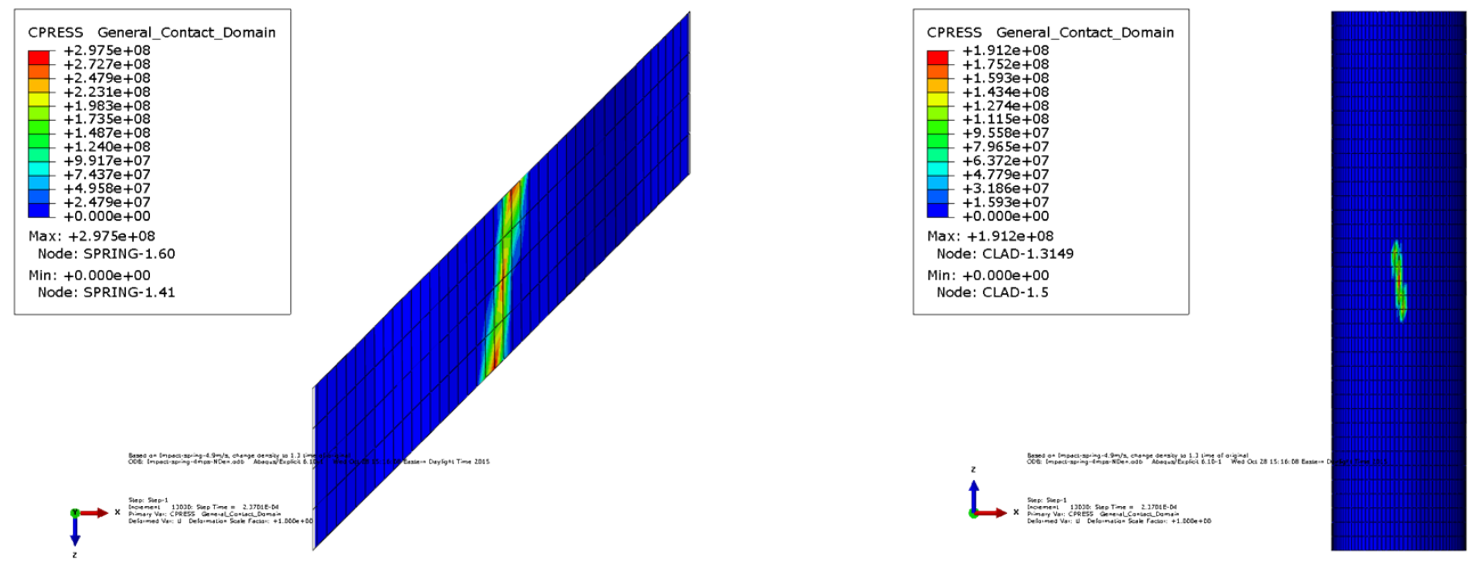

Fig. 57. Contact pressure on the spring and cladding during the fuel rod's impact on the spring at the initial velocity of $4.9 \mathrm{~m} / \mathrm{s}$ 